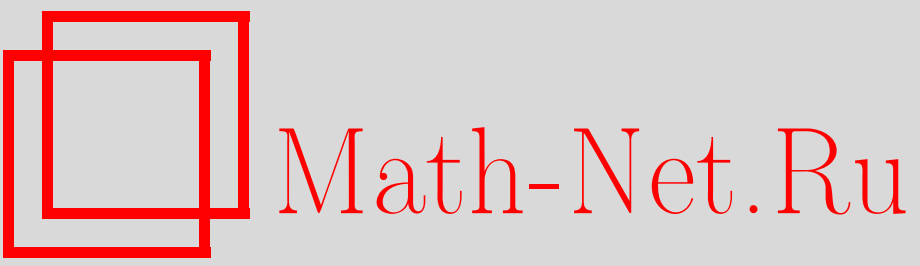

А. Д. Миронов, Групповой подход к $\tau$-функции и ее квантованию, ТМФ, 1998 , том 114, номер 2, 163-232

DOI: https://doi.org/10.4213/tmf836

Использование Общероссийского математического портала Math-Net.Ru подразумевает, что вы прочитали и согласны с пользовательским соглашением

http://www . mathnet.ru/rus/agreement

Параметры загрузки:

IP : 35.173 .219 .149

26 апреля 2023 г., $12: 14: 44$ 
ТЕОРЕТИЧЕСКАЯ

И МАТЕМАТИЧЕСКАЯ

ФИЗИКА

Том 114, № 2

февраль, 1998

(C) $\mathbf{1 9 9 8}$ г.

А. Д. Миронов*

\section{ГРУППОВОЙ ПОДХОД \\ К $\tau$-ФУНКЦИИ И ЕЕ КВАНТОВАНИЮ ${ }^{1)}$}

Работа представляет собой обзор результатов, связанных с обобщением понятий $\tau$-функции и интегрируемых иерархий, и их интерпретации на теоретико-групповом языке, допускающем непосредственное проведение процедуры квантования. Подробно обсуждаются различные теоретико-групповые структуры, ассоциированные с данной интегрируемой системой, и их деформация при квантовании.

\section{Содержание}

1. Введение

2. Фермионное представление интегрируемых иерархий

2.1. Общее фермионное представление стандартных интегрируемых иерархий

2.2. Билинейные тождества для классической иерархии $2 \mathrm{PT}$

2.3. Фермионное представление полубесконечных иерархий

3. Теоретико-групповые структуры молекулы Тоды

4. Обобщенная $\tau$-функция и билинейные тождества

4.1. $\tau$-Функция и билинейные тождества

4.2. Пример. Квантовая группа $S L_{q}(2)$

4.3. Универсальный Т-оператор (групповой элемент)

4.4. $\tau$-Функция и представления алгебры функций

4.5. Разностная иерархия $\mathrm{KOC}$

5. Квантовая и классическая иерархии КП с различными эволюциями

5.1. Структура фундаментальных представлений

5.2. Параметризации группового элемента в фундаментальных представлениях

5.3. Классическая $(q=1)$ иерархия КП/Тоды в разных параметризациях

5.4. Квантовый случай $(q \neq 1)$

6. Волновая функция и $S$-матрица в квантовой теории Лиувилля (Тоды)

6.1. Классическая система Тоды как гамильтонова редукция

6.2. Квантовая механика Лиувилля, обшая схема решения

\footnotetext{
1) Статья написана по заказу Редколлегии.
}

* Отделение теоретической физики Физического института РАН; Институт теоретической и экспериментальной физики, Москва, Россия. E-mail: mironov@lpi.ac.ru, mironov@itep.ru 
6.3. Квантовая механика Лиувилля, группа $S L(2, \mathbf{R})$

6.4. Квантовая механика Тоды

Приложение 1. Полубесконечные иерархии

Приложение 2. Фундаментальные представления группы $S L_{q}(p)$

Приложение 3. Явный пример: $\tau_{2}$ для $S L_{q}(p)$

Приложение 4. Построение функции Уиттекера для группы $S L(p)$

П4.1. Обозначения

П4.2. Представления

П4.3. Гамильтониан и уравнение Шредингера

П4.4. Решение уравнения Шредингера

\section{1. ВВЕ ДЕНИЕ}

Явление интегрируемости в последние годы играет все большую и большую роль в физике частиц и теории струн $[1,2]$. Один из основных уроков, которому учат многочисленные конкретные примеры, состоит в том, что явление классической интегрируемости в квантовы $x$ теориях поля оказьвается весьма обшим.

Одним из первых примеров использования именно интегрируемых систем в указанной области теоретической физики явились попытки пертурбативных деформаций конформных теорий [3-5] в "интегрируемых" направлениях. Изучение деформаций, вообще говоря, необходимо для описания непертурбативных решений теории струн. Конечно, пертурбативные деформации конформных теорий дали только описание окрестности критических точек, что не прояснило глобальной структуры конфигурационного пространства теории струн. Однако уже в работах [3-5] (см. также $[6,7])$ отмечалась важность именно интегрируемых деформаций конформных теорий. Более того, и в непосредственном контексте теории струн ожидалось, что сумма всего пертурбативного ряда для амплитуд (или статистической суммы) описывается некоторой квантовой интегрируемой системой $[8,9]$. Здесь важно, что эффективное действие для квантовой интегрируемой системы является $\tau$-функцией классической интегрируемой системы ${ }^{2}$. Это удивительное явление уже встречалось в ряде простых примеров [13-16], хотя никогда не было объяснено.

Интересно, что имеющиеся примеры могут быть разделены на два класса: когда классическая интегрируемая система естественно зависит от времен (констант связи) и когда от так называемых переменных Мивы - собственных значений некоторой бесконечной внешней матрицы. Примером первого типа является некоторый функционал корреляторов квантового уравнения Шредингера, который оказывается $\tau$-функцией классического интегрируемого нелинейного уравнения того же типа [13]. Типичный пример второго типа - статистическая сумма шестивершинной модели с нетривиальными граничными условиями, которая оказывается $\tau$-функцией двумеризованной цепочки Тоды в переменных Мивы (которые суть граничные условия) [16].

Однако наиболее содержательным примером рассматриваемого явления интегрируемости являются матричные модели [2, 17-19]. В частности, в матричных моделях реа-

\footnotetext{
2) Достаточно интересно, что интегрирование некоторых объектов типа $\tau$-функции $[10,11]$ по универсальному пространству модулей в теории струн [12] приводит опять к $\tau$-функции, заданной на том же универсальном пространстве модулей.
} 
лизуются оба упомянутых выше типа переменных: именно, статистическая сумма дискретных матричных моделей есть $\tau$-функция с временами, являющимися константами связи, а непрерывных - $\tau$-функция, зависяшая от внешней матрицы, задаюшей переменные Мивы.

В действительности явление классической интегрируемости эффективной теории оказывается весьма общим и не специфичным, скажем, для двух измерений. Так, ярким примером является решение четырехмерной $N=2$ суперсимметричной теории Янга-Миллса, найденное недавно Виттеном и Зайбергом [20, 21], классические интегрируемые структуры которого были выявлены в работе [22] (для ознакомления с последними достижениями в этой области см. $[1,23,24]$ и приведенные там ссылки). Вследствие такой общности этого явления можно было бы ожидать, что эффективное действие в типичных теориях поля бывает интегрируемым при правильном выборе переменных и, конечно, учете всех возможных взаимодействий. На практике набор этих последних обычно ограничен некоторыми дополнительными соображениями (перенормируемость и т.д.).

Как уже говорилось, хотя явление интегрируемости в теориях поля и теории струн было исследовано в ряде примеров, его источник остается до сих пор не понятым. В настояшей работе обсуждаются вопросы, связанные с обшими свойствами квантовых и классических $\tau$-функций, что должно помочь пролить некоторьй свет на указанньй феномен. Кроме того, здесь же исследуются некоторые непосредственные приложения общего подхода, например вычисление $S$-матрицы квантовой теории Лиувилля.

Для того чтобы обсуждать, каким образом корреляторы квантовой интегрируемой системы связаны с классическими иерархиями, надо вначале переформулировать квантовую систему в терминах, адекватных этим классическим иерархиям. Ключевым объектом в этих иерархиях является $\tau$-функция, в то время как квантовые интегрируемые системы до сих пор формулировались исключительно в терминах $R$-матрицы [25-30]. Поэтому основной задачей настоящей работы является обобщение понятия $\tau$-функции таким образом, чтобы оно включало в себя квантовый случай. Для решения этой задачи мы собираемся подробно обсудить само понятие $\tau$-функции и, в частности, выделить различные теоретико-групповые (симметрийные) аспекты, связанные с ним. На этом пути естественно вводится понятие обобщенной $\tau$-функции, которая все еще может быть описана чисто алгебраически и удовлетворяет набору билинейных тождеств типа уравнений Хироты.

Необходимость обобщения понятия $\tau$-функции трудно переоценить. Начнем с того, что уже упоминавшиеся матричные модели, отвечаюшие стандартным интегрируемым иерархиям, являются только простейшими моделями. Более сложные и важные физические примеры описьвают обобщенную модель Концевича-Казакова-Мигдала [31], многомерные калибровочные теории [32], теории двумерной гравитации, взаимодействуюшие с конформной материей при $c>1$ (т.е. отвечаюшие размерности пространства-времени $d>2$ снова многомерные модели) и т.д. Все эти примеры отвечают моделям, в которых угловые переменные не могут быть отфакторизованы и затем проинтегрированы. Таким образом, они должны описываться более сложными "обобщенными" иерархиями.

Другой важньй класс “обобщенных" интегрируемых иерархий должен отвечать тео- 
риям Весса-Зумино-Виттена-Новикова (ВЗВН) уровня, большего чем 1. Дальнейшее развитие в том же направлении должно привести к описанию 2-петлевых, 3-петлевых и т.д. алгебр в рамках интегрируемых иерархий. Все рассмотренные примеры отвечают так называемым некартановским иерархиям [17].

Еще одной важной задачей, которая должна решаться на пути квантования обобщенной $\tau$-функции, является аккуратная регуляризация теории Лиувилля [33]. К этой проблеме, однако, имеются и другие подходы, которые будут частично описаны ниже.

Сразу оговоримся, что мы не обсуждаем вопрос, почему проводимая в работе $q$-деформация $\tau$-функции отвечает, действительно, квантованию системы, т.е. построению квантовой теории поля (квантовой механики) вместо классической. Вместо этого мы просто отсылаем читателя к работам [25-30], объясняющим, почему переход к квантовым группам и квантовым $R$-матрицам отвечает квантованию классических систем.

Как уже говорилось, основным инструментом при исследовании обобщенных $\tau$-функций является теория групп. Точнее, при использовании различных симметрийных структур интегрируемых иерархий их удается сформулировать на чисто алгебраическом языке. Как обычно в теоретической физике, именно симметрийный подход позволяет устанавливать самые общие закономерности, не зависящие от деталей динамики. В частности, именно общая симметрийная структура различных эффективных действий квантовой теории поля должна объяснять их типичную интегрируемую структуру. Поэтому важно прояснить различные алгебраические структуры и их роль при квантовании теории.

В качестве типичного примера в данной работе, являющейся обзором статей [34-40], рассматривается интегрируемая система типа Тоды (или Лиувилля как частный пример этой последней). В такой системе, как показано в разделах 3 и 6 , имеются три различные групповые структуры. Две из них связаны с действием группы в пространстве спектрального параметра: одна - это группа $g l(\infty)$, грассманниан которой параметризует все решения двумеризованной иерархии Тоды обшего вида, другая - группа $s l(n)$, описываюшая редукцию всего этого большого пространства решений к решениям $s l(n)$-Тоды. Таким образом, обе эти группы действуют на решениях интегрируемой иерархии. Именно эти группы заменяются своими квантовыми аналогами при квантовании системы.

Однако процедура квантования не является механической, не любая групповая структура должна быть обязательно проквантована. Действительно, в системе Лиувилля имеется еще одна группа. Она действует в "пространстве-времени", т.е. на временах интегрируемой системы. При квантовании, однако, эта группа остается классической, меняется только ее трактовка - квантование вводит орбитную интерпретацию. Действительно, если классическая система получается гамильтоновой редукцией свободной системы, заданной на кокасательном расслоении к простой вещественной группе Ли [41-43], то квантовая модель связана с неприводимыми унитарными представления той же самой группы. Таким образом, квантовая система должна скорее интерпретироваться в рамках геометрического квантования [44]. Изучению этой групповой структуры посвящен раздел 6. В частности, в этом разделе показано, что методы теории групп позволяют не только построить волновую функцию квантовой теории Тоды (назьваемую функцией Уиттекера) [45-48], но и найти ее асимптотики [49, 50] и, следовательно, 
$S$-матрицу (или двухточечный коррелятор).

Вычисления такого сорта были известны довольно давно [51], однако они были проделаны в рамках разложения Ивасавы и плохо обобщаются на аффинный случай. В разделе 6 мы представляем другое вычисление, основанное на разложении Гаусса. Достоинством этого метода является его непосредственная обобшаемость на лиувиллевскую теорию поля, которой отвечает аффинная алгебра (в этом случае следует использовать "поточечное" разложение Гаусса, аналогичное применяемому при бозонизации теорий ВЗВН [52]). Результаты вычисления волновой функции и ее асимптотик легко продолжаются на этот (афффинный) случай, приводяший к $S$-матрище двумерной теории Лиувилля. Эта $S$-матрица совпадает с $S$-матрицей, полученной недавно Замолодчиковыми [53] и Дорном и Отто [54, 55].

Другие групповые структуры, которые должны заменяться $q$-деформированными аналогами при квантовании интегрируемой системы, обсуждаются в остальной части статьи. В частности, в разделе 4 исследуется обобщение $\tau$-функции, допускающее непосредственное квантование. Естественно попытаться построить такую квантовую $\tau$-функцию, чтобы она оставалась $c$-числовой. В пункте 4.5 показано, что такого рода деформацию классической иерархии, действительно, можно проделать, причем результируюшая иерархия из дифференциальной превратится в разностную. Однако эта деформация оказывается слишком тривиальной, поскольку сводится к (весьма сложному) переопределению времен первоначальной иерархии. Таким образом, остается задача построения нетривиальной деформации понятия $\tau$-функции (которая приводит к некоммутативному объекту).

Эту задачу удается решить на чисто алгебраическом пути, в том же разделе $4 \tau$-функция определяется по представлению любой алгебры как производящая функция ее матричных элементов в некотором (произвольном) представлении. Так, определенная $\tau$-функция удовлетворяет системе билинейных тождеств (БТ). В классическом случае эти БТ дифференциальные. Для квантования иерархии достаточно вместо классической группы взять квантовую. При этом дифференциальные БТ становятся разностными, а сама $\tau$-функция - оператором.

Следует заметить, что $\tau$-функция, определенная по произвольному представлению алгебры, приводит к БТ, заданным некоммутирующими потоками, т.е. в стандартном смысле теория перестает быть интегрируемой (по Лиувиллю). Она, однако, сохраняет множество важнейших свойств интегрируемых теорий и отвечает некартановским иерархиям [17], упоминавшимся вьше.

Для того чтобы получить $\tau$-функцию, отвечаюшую стандартной иерархии Кадомцева-Петвиашвили (КП) или Тоды, нужно рассмотреть фундаментальные представления группы $S L(\infty)$. Для таких представлений возможна некоторая специальная редукция обшей $\tau$-функции, которая приводит к обычным интегрируемым иерархиям.

Следуюшая проблема - задача построения квантового аналога иерархии КП. Эта задача Является крайне нетривиальной, поскольку указанная специальная редукция общей $\tau$-функции, возможная в фундаментальных представлениях, разрушается при квантовании. Удается, однако, построить квантование некоторой новой классической иерархии, которая является, по сути, иерархией КП, но с необычной эволюцией. Это продемонстрированно в разделе 5 . 
Для большей стройности изложения часть материала, носящего более технический характер, вынесена в приложения. Некоторые дополнительные детали, оставшиеся вне рамок настоящего обзора, могут быть найдены в оригинальных статьях [34-40] и, особенно, в статье [39], результаты которой будут здесь рассмотрены весьма сжато.

Автор благодарен А. Герасимову, С. Харчеву, С. Хорошкину, Д. Лебедеву, А. Маршакову, А. Морозову, М. Ольшанецкому и Л. Винэ за участие в совместных работах и полезные обсуждения и Г. Вайгту, А. Горскому, В. Добреву, А. Забродину, Ал. Замолодчикову, В. Корепину, Н. Некрасову, Н. Славнову, И. Тютину и Й. Шниттге за полезные обсуждения. Работа частично поддержана грантами РФФИ-96-01-01106 и INTAS-97-1038.

\section{2. ФЕРМИОННОЕ ПРЕДСТАВЛЕНИЕ ИНТЕГРИРУЕМЫХ ИЕРАРХИЙ}

Вначале мы изложим вкратце общее фермионное описание стандартных интегрируемых иерархий, следуя в основном работам киотской школы [56-63] (см.также [64, 65]), и опишем, как на этом языке выглядит важный частный случай - полубесконечные иерархии ${ }^{3)}$. Заметим, что фермионный подход - самый универсальный язык, до сих пор применявшийся для описания интегрируемых систем, поскольку он отвечает формальному грассманниану (грассманниан Сато [66]), сформулированному в терминах формальных рядов. Хотя имеюшиеся альтернативные подходы (см., например, [67-69]) лучше описывают, скажем, конечнозонные решения [70], именно грассманниан Сато необходим для работы с сингулярными точками (описывающими, например, матричные модели, см. [71]).

\section{1. Общее фермионное представление стандартных интегрируемых иерархий}

Мы начнем с описания иерархии двумеризованной решетки Тоды (2PТ), $\tau$-функция которой задается корреляторами в теории свободных фермионов $\psi, \tilde{\psi}(b, c$-система со спином 1/2). А именно, она определена как отношение корреляторов ${ }^{4)}$

$$
\tau_{n}(t, \bar{t} \mid G) \equiv \frac{\left\langle n\left|e^{H} G e^{\bar{H}}\right| n\right\rangle}{\langle n|G| n\rangle}
$$

в теории двумерных свободных фермионных полей $\psi(z), \psi^{*}(z)$ :

$$
\begin{gathered}
\psi(z)=\sum_{\mathbf{Z}} \psi_{n} z^{n}, \\
\psi^{*}(z)=\sum_{\mathbf{Z}} \psi_{n}^{*} z^{-n-1}, \\
\left\{\psi_{k}, \psi_{m}^{*}\right\}=\delta_{k m}, \quad\left\{\psi_{k}, \psi_{m}\right\}=\left\{\psi_{k}^{*}, \psi_{m}^{*}\right\}=0 .
\end{gathered}
$$

\footnotetext{
3) Именно эти полубесконечные иерархии описывают, в частности, матричные модели.

4) В дальнейшем тексте мы будем опускать из определения $\tau$-функции коррелятор, служащий нормировочным множителем, всюду, где это не может привести к недоразумениям, т.е. он не сингулярен.
} 
В определении (2.1) использованы следующие обозначения:

$$
H=\sum_{k>0} t_{k} J_{k}, \quad \bar{H}=\sum_{k>0} \bar{t}_{k} J_{-k},
$$

где токи определены как

$$
J(z)=\psi^{*}(z) \psi(z), \quad \text { т.е. } \quad J_{k}=\sum_{\mathbf{z}} \psi_{i} \psi_{i+k}^{*}
$$

Величина $G$ задается формулой

$$
G=: \exp \left\{\sum_{m, n} \mathcal{G}_{m n} \psi_{m}^{*} \psi_{n}\right\}:
$$

и является элементом группы $G L(\infty)$ (или группы Клиффорда), реализованным в бесконечномерном грассманниане (см. также эквивалентные описания в [67-69]). Нормальное упорядочивание должно пониматься здесь как упорядочивание по отношению к вакууму $|0\rangle$, который определен условиями

$$
\psi_{m}|0\rangle=0, \quad m<0 ; \quad \psi_{m}^{*}|0\rangle=0, \quad m \geq 0
$$

Можно рассмотреть и более общее нормальное упорядочивание, определенное по отношению к вакууму $|k\rangle$, который задан условиями

$$
\psi_{m}|k\rangle=0, \quad m<k ; \quad \psi_{m}^{*}|k\rangle=0, \quad m \geq k .
$$

Конкретное решение иерархии ( $\tau$-функция $(2.1))$ зависит только от выбора элемента $G$ (или эквивалентно может быть определено единственным образом матрицей $\mathcal{G}_{k m}$ ). Замечательным достижением киотской школы является утверждение о том, что любое решение иерархии 2РТ может быть задано некоторым элементом $G$ вида (2.6) и наоборот каж дьй такой элемент $G$ отвечает некоторому решению иерархии $2 \mathrm{PT}$.

Заметим, что в рамках описываемого здесь фермионного подхода [56-63] 2РТ является однокомпонентной иерархией наиболее обшего вида. Скажем, иерархия КП получается из нее, если приравнять нулю все отрицательные времена и нулевое время или просто как-либо их фиксировать и не рассматривать соответствующие эволюционные уравнения. В этом смысле иерархия КП - это подиерархия 2РТ, а не редукция, и описывается она тем же множеством элементов $G$, но меньшим числом эволюционных потоков.

Другим примером является цепочка Тоды, это уже редукция, которая получается из 2РТ при наложении специального условия на элемент грассманниана. В результате $\tau$-функция цепочки Тоды оказьвается зависящей не от положительных и отрицательных времен по отдельности, а только от их суммы [64 $]^{5)}$ (поэтому без потери общности можно использовать эволюцию только по одному набору времен). Такое свойство цепочки Тоды может быть взято за ее определение.

\footnotetext{
${ }^{5)}$ В большинстве статей цепочка Тоды зависит только от разности времен. Это связано с противоположным знаком, использованным в $(2.1)$ при $\bar{H}$.
} 
Теперь мы получим несколько свойств описанной фермионной системы, полезных для дальнейшего. Из коммутационных соотношений (2.3) можно легко найти, как преобразуются фермионные моды при действии на них элемента грассманниана (2.6):

$$
G \psi_{k} G^{-1}=\psi_{j} R_{j k}, \quad G \psi_{k}^{*} G^{-1}=\psi_{j}^{*} R_{k j}^{-1},
$$

где матрица $R_{j k}$ может быть выражена через $\mathcal{G}_{j k}$ (см. [14]). Ниже мы увидим, что $R_{j k}$ является важным строительным блоком в детерминантных представлениях $\tau$-функций.

Введем еше некоторые обозначения. Используя $(2.3)$, определяем эволюцию $\psi(z)$ и $\psi^{*}(z)$ по временам $\left\{t_{k}\right\},\left\{\bar{t}_{k}\right\}$ :

$$
\begin{aligned}
\psi(z, t) & \equiv e^{H(t)} \psi(z) e^{-H(t)}=e^{\xi(t, z)} \psi(z), \\
\psi^{*}(z, t) & \equiv e^{H(t)} \psi^{*}(z) e^{-H(t)}=e^{-\xi(t, z)} \psi^{*}(z), \\
\psi(z, \bar{t}) & \equiv e^{\bar{H}(\bar{t})} \psi(z) e^{-\bar{H}(\bar{t})}=e^{\xi\left(\bar{t}, z^{-1}\right)} \psi(z), \\
\psi^{*}(z, \bar{t}) & \equiv e^{\bar{H}(\bar{t})} \psi^{*}(z) e^{-\bar{H}(\bar{t})}=e^{-\xi\left(\bar{t}, z^{-1}\right)} \psi(z),
\end{aligned}
$$

где

$$
\xi(t, z)=\sum_{k=1}^{\infty} t_{k} z^{k}
$$

Находим также полиномы Шура по формуле

$$
\exp \left\{\sum_{k>0} t_{k} x^{k}\right\} \equiv \sum_{k>0} P_{k}\left(t_{k}\right) x^{k}, \quad P_{k}\left(t_{k}\right)=0, \quad k<0 .
$$

Используя это определение и (2.10)-(2.13), получаем эволюцию фермионных мод:

$$
\begin{aligned}
& \psi_{k}(t) \equiv e^{H(t)} \psi_{k} e^{-H(t)}=\sum_{m=0}^{\infty} \psi_{k-m} P_{m}(t), \\
& \psi_{k}^{*}(t) \equiv e^{H(t)} \psi_{k}^{*} e^{-H(t)}=\sum_{m=0}^{\infty} \psi_{k+m}^{*} P_{m}(-t), \\
& \psi_{k}(\bar{t}) \equiv e^{\bar{H}(\bar{t})} \psi_{k} e^{-\bar{H}(\bar{t})}=\sum_{m=0}^{\infty} \psi_{k+m} P_{m}(\bar{t}), \\
& \psi_{k}^{*}(\bar{t}) \equiv e^{\bar{H}(\bar{t})} \psi_{k}^{*} e^{-\bar{H}(\bar{t})}=\sum_{m=0}^{\infty} \psi_{k-m}^{*} P_{m}(-\bar{t}) .
\end{aligned}
$$

Теперь мы можем вывести детерминантное представление для $\tau$-функции ${ }^{6)}$. Для этого определим совершенно заполненное вакуумное состояние $|-\infty\rangle$, которое удовлетворяет требованию

$$
\psi_{i}^{*}|-\infty\rangle=0, \quad i \in \mathbf{Z}
$$

\footnotetext{
6) Нижеследующий вывод, конечно, требует при реальных вычислениях определенных свойств сходимости, в частности, определителей бесконечномерных матриц. Скажем, достаточным является рассматривать матрицы с конечным числом отличных от нуля диагональных элементов [62]. Иногда, однако, этот класс матриц оказывается слишком ограничительным, как, например, в матричных моделях $[71,72]$. Обсуждаемые же ниже формулы являются следствием некоторых соотношений, которые можно проверить в формальных рядах, и потому они верны всегда, когда свойства сходимости выполнены. По той же причине мы не обсуждаем ниже вопроса существования совершенно заполненного вакуумного состояния.
} 
Тогда любой "сдвинутый" вакуум получается из него следующим образом:

$$
|n\rangle=\psi_{n-1} \psi_{n-2} \ldots|-\infty\rangle .
$$

Заметим, что действие любого элемента $G$ группы Клиффорда (и, как следствие, действие $\left.e^{\bar{H}(\bar{t})}\right)$ на $|-\infty\rangle$ тривиально: $G|-\infty\rangle \sim|-\infty\rangle$. Поэтому с помощью $(2.17)$ и $(2.18)$ получаем из (2.1)

$$
\begin{aligned}
\tau_{n}(t, \bar{t}) & =\left\langle-\infty\left|\ldots \psi_{n-2}^{*}(-t) \psi_{n-1}^{*}(-t) G \psi_{n-1}(\bar{t}) \psi_{n-2}(\bar{t}) \ldots\right|-\infty\right\rangle \sim \\
& \left.\sim \operatorname{det}\left[\left\langle-\infty\left|\psi_{i}^{*}(-t) G \psi_{j}(\bar{t}) G^{-1}\right|-\infty\right\rangle\right]\right|_{i, j \leq n-1} .
\end{aligned}
$$

Используя (2.9), находим

$$
G \psi_{j}(\bar{t}) G^{-1}=\sum_{m, k} P_{m}(\bar{t}) \psi_{k} R_{k, j+m},
$$

поэтому “явное" решение иерархии $2 \mathrm{PT}$ может быть записано в детерминантной форме

$$
\tau_{n}(t, \bar{t}) \sim \operatorname{det}_{i, j<0} C_{i+n, j+n}(t, \bar{t}),
$$

где $\mathrm{e}^{7)}$

$$
C_{i j}(t, \bar{t})=\sum_{k, m} R_{k m} P_{k-i}(t) P_{m-j}(\bar{t}) .
$$

Зависимость от времени матричных элементов (2.25) в детерминанте (2.24) задана следуюшими уравнениями:

$$
\begin{array}{ll}
\partial C_{i j} / \partial t_{p}=C_{i, j-p}, & j>p>0, \\
\partial C_{i j} / \partial \bar{t}_{p}=C_{i-p, j}, & i>p>0,
\end{array}
$$

которые являются прямым следствием соответствуюшего свойства полиномов Шура:

$$
\partial P_{k} / \partial t_{p}=P_{k-p},
$$

следуюшего непосредственно из их определения.

Как уже отмечалось, иерархия КП отвечает эволюции только по положительным временам $\left\{t_{k}\right\}$; отрицательные времена $\left\{\bar{t}_{k}\right\}$ играют роль параметров, задающих семейство точек в грассманниане, и могут быть поглошены переопределением матришы $R_{k m}$. Тогда $\tau$-функция (модифишированной) иерархии КП имеет вид

$$
\tau_{n}(t)=\left\langle n\left|e^{H(t)} G(\bar{t})\right| n\right\rangle \sim \operatorname{det}_{i, j<0}\left[\sum_{k} R_{k, j+n}(\bar{t}) P_{k-i-n}(t)\right],
$$

где $G(\bar{t}) \equiv G e^{\bar{H}(\bar{t})}$ и

$$
R_{k j}(\bar{t}) \equiv \sum_{m} R_{k m} P_{m-j}(\bar{t})
$$

\footnotetext{
7) Заметим, что мы нигде не выписываем области суммирования, поскольку они автоматически выделяются свойством (определением) полиномов Шура $P_{k}=0$ при $k<0$ в $(2.15)$.
} 


\section{2. Билинейные тождества для классической иерархии $2 \mathrm{PT}$}

Для дальнейшего нам понадобятся еще следующие две важнейшие формулы теории интегрируемых систем [58]:

$$
\begin{aligned}
\langle n| \psi(z) \exp [H(t)] & =z^{n-1}\langle n-1| \exp \left[H\left(t-\epsilon\left(z^{-1}\right)\right)\right] \equiv \\
& \equiv z^{n-1} \widehat{X}(z, t)\langle n-1| e^{H(t)}, \\
\langle n| \psi^{*}(z) \exp [H(t)] & =z^{-n}\langle n+1| \exp \left[H\left(t+\epsilon\left(z^{-1}\right)\right)\right] \equiv \\
& \equiv z^{-n} \widehat{X}^{*}(z, t)\langle n+1| e^{H(t)},
\end{aligned}
$$

где

$$
\begin{aligned}
\widehat{X}(z, t) & \equiv e^{\xi(z, t)} e^{-\xi\left(z, \tilde{\partial}_{t}\right)}, \\
\widehat{X}^{*}(z, t) & \equiv e^{-\xi(z, t)} e^{\xi\left(z, \tilde{\partial}_{t}\right)}
\end{aligned}
$$

и, как обычно, $\tilde{\partial}_{t} \equiv\left(\partial_{t_{1}},(1 / 2) \partial_{t_{2}}, \ldots\right)$.

Используя эти формулы, можно выразить функцию Бейкера-Ахиезера (ФБА) иерархии КП, определенную через $\tau$-функцию, выражением [58]

$$
\Psi\left(\mu \mid t_{n}\right)=e^{\Sigma t_{n} \mu^{n}} \frac{\tau_{0}\left(t_{n}-\frac{\mu^{-n}}{n} \mid \bar{t}_{n}\right)}{\tau_{0}\left(t_{n} \mid \bar{t}_{n}\right)}
$$

через фермионный коррелятор

$$
\Psi\left(\mu \mid t_{n}\right)=\frac{\left\langle 1\left|e^{H(t)} \psi(\mu) G\right| 0\right\rangle}{\left\langle 0\left|e^{H(t)} G\right| 0\right\rangle} .
$$

Аналогично можно рассмотреть сопряженную ФБА, заданную определением

$$
\Psi^{*}\left(\mu \mid t_{n}\right)=\frac{\left\langle 1\left|e^{H(t)} \psi^{*}(\mu) G\right| 0\right\rangle}{\left\langle 0\left|e^{H(t)} G\right| 0\right\rangle} .
$$

Рассмотрев ненулевой вакуум, можно ввести и более общую пару ФБА, зависящих от нулевого времени $n$.

Аналогично в случае полной иерархии $2 \mathrm{PT}$ можно рассмотреть четыре различные $\Phi Б А$, поскольку можно вставлять фермионы и справа от элемента грассманниана $G$.

Выведем теперь БТ, которым удовлетворяет $\tau$-функция иерархии 2РТ $(2.1)[62,64]$. Для этого заметим, что благодаря соотношениям (2.9) тензорное произведение элементов грассманниана $G \otimes G(2.6)$ коммутирует с тензорным произведением $\Gamma \equiv \sum_{i} \psi_{i} \otimes \psi_{i}^{*}$. Чтобы сделать обозначения удобными для дальнейшего изложения, мы далее обозначаем фермионные моды $\psi_{k}$ через $\psi_{k}^{+}$и $\psi_{k}^{*}$ через $\psi_{k}^{-}$.

Изучим теперь матричные элементы тождества

$$
\Gamma(G \otimes G)=(G \otimes G) \Gamma
$$


между состояниями $\langle n+1| U(t) \otimes\langle m-1| U\left(t^{\prime}\right)$ и $\bar{U}(\bar{t})|n\rangle \otimes \bar{U}\left(\bar{t}^{\prime}\right)|m\rangle$, где мы обозначили $U(t) \equiv e^{H(t)}$ и $\bar{U}(\bar{t}) \equiv e^{\bar{H}(\bar{t})}$ :

$$
\begin{aligned}
\sum_{i} & \left\langle n+1\left|U(t) \psi_{i}^{+} G \bar{U}(\bar{t})\right| n\right\rangle \cdot\left\langle m-1\left|U\left(t^{\prime}\right) \psi_{i}^{-} G \bar{U}\left(\bar{t}^{\prime}\right)\right| m\right\rangle= \\
& =\sum_{i}\left\langle n+1\left|U(t) G \psi_{i}^{+} \bar{U}(\bar{t})\right| n\right\rangle \cdot\left\langle m-1\left|U\left(t^{\prime}\right) G \psi_{i}^{-} \bar{U}\left(\bar{t}^{\prime}\right)\right| m\right\rangle
\end{aligned}
$$

Теперь можно переписать (2.39) с помошью полей свободных фермионов $(2.2)^{8)}$ :

$$
\begin{aligned}
& \oint_{\infty} d z\left\langle n+1\left|U(t) \psi^{+}(z) G \bar{U}(\bar{t})\right| n\right\rangle \cdot\left\langle m-1\left|U\left(t^{\prime}\right) \psi^{-}(z) G \bar{U}\left(\bar{t}^{\prime}\right)\right| m\right\rangle= \\
& =\oint_{0} d z\left\langle n+1\left|U(t) G \psi^{+}(z) \bar{U}(\bar{t})\right| n\right\rangle \cdot\left\langle m-1\left|U\left(t^{\prime}\right) G \psi^{-}(z) \bar{U}\left(\bar{t}^{\prime}\right)\right| m\right\rangle .
\end{aligned}
$$

Если обобщить определения ФБА (2.36) и (2.37) на ненулевой вакуум и ввести отрицательные времена

$$
\Psi_{n}^{ \pm, i} \equiv\left\langle n \pm 1\left|\widehat{U}(t) \psi_{i}^{ \pm} G \widehat{\bar{U}}(\bar{t})\right| n\right\rangle,
$$

то эти формулы могут быть записаны в более компактном виде (здесь и далее мы используем обозначение $t$ как для положительных, так и для отрицательных времен там, где это не может привести к непониманию):

$$
\sum_{i} \Psi_{k}^{+, i}(t) \Psi_{l}^{-, i}\left(t^{\prime}\right)=\sum_{j} \bar{\Psi}_{k+1}^{+, j}(\bar{t}) \bar{\Psi}_{l-1}^{+, j}\left(\bar{t}^{\prime}\right) .
$$

Здесь $\bar{\Psi}$ задает вторую пару ФБА, определенных аналогичным образом, но с фермионом, стоящим справа от элемента $G$.

Эти ФБА генерируются действием вершинных операторов (2.33), (2.34) (см. $(2.31),(2.32))$ :

$$
\sum_{i} \Psi_{k}^{+, i}(t) z^{i} \equiv \widehat{X}^{+}(z, t) \tau_{n}(t), \quad \sum_{i} \Psi_{k}^{-, i}(t) z^{-i-1} \equiv \widehat{X}^{-}(z, t) \tau_{n}(t)
$$

и аналогично для $\widehat{\bar{X}}^{ \pm}(z, t)$. Тогда $(2.39)$ может быть переписано в виде

$$
\begin{aligned}
\oint_{\infty} d z & \widehat{X}^{-}(z, t) \tau_{n}(t, \bar{t}) \widehat{X}^{+}\left(z, t^{\prime}\right) \tau_{m}\left(t^{\prime}, \bar{t}^{\prime}\right)= \\
& =\oint_{0} d z \widehat{\widehat{X}}^{-}(z, \bar{t}) \tau_{n+1}(t, \bar{t}) \widehat{\widehat{X}}^{+}\left(z, \bar{t}^{\prime}\right) \tau_{m-1}\left(t^{\prime}, \bar{t}^{\prime}\right)
\end{aligned}
$$

Эта интегральная форма БТ превращается в бесконечный набор уравнений (см. также (3.4)), если проделать разложение по степеням $t-t^{\prime}$ и $\bar{t}-\bar{t}^{\prime}$ :

$$
\begin{gathered}
\sum_{i=0}^{\infty} P_{i}(-2 y) P_{i+1}\left(\widetilde{D}_{t}\right) \exp \left\{\left[\sum_{i} y_{i} D_{t_{i}}\right]\right\} \tau \cdot \tau=0 \\
\tau_{n} \partial_{t_{1}} \partial_{\bar{t}_{1}} \tau_{n}-\partial_{t_{1}} \tau_{n} \partial_{\bar{t}_{1}} \tau_{n}=\tau_{n+1} \tau_{n-1}
\end{gathered}
$$

\footnotetext{
8) Контур интегрирования в этой формуле выбирается в зависимости от того, по положительным или отрицательным степеням $z$ раскладывается подынтегральное выражение.
} 
где $D_{t}$ - символы Хироты, действуюшие на произведение двух функций по следуюшему правилу:

$$
\left.D_{x}^{k}(f \cdot g) \equiv \partial_{y}[f(x+y) g(x-y)]\right|_{y=0}, \quad \widetilde{D} \equiv\left(D_{t_{1}}, \frac{1}{2} D_{t_{2}}, \ldots\right),
$$

$P_{i}-$ полиномы Шура и разложение по произвольному набору параметров $\left\{y_{k}\right\}$ задает бесконечный набор уравнений иерархий КП/Тоды.

\section{3. Фермионное представление полубесконечных иерархий}

Теперь мы применим описанный формализм к случаю полубесконечных иерархий, в частности, получим детерминантные формулы $[73,65]$. Ключевое отличие формул типа (2.24) от детерминантных представлений, которые будут получены в данном пункте, в том, что в полубесконечных иерархиях возникают конечные детерминанты. Это очень важное отличие, возникающее благодаря специфическому граничному (с точки зрения цепочки частиц) условию

$$
\tau_{0}=1
$$

Это условие может служить определением полубесконечных иерархий [74], которые изучались в работах $[73,65]$. Оказывается, полубесконечные иерархии описываются сингулярными элементами грассманниана, которые содержат только положительные (или только отрицательные) фермионные моды. Это, в свою очередь, подразумевает четверть-бесконечную матрицу $\mathcal{G}_{m n}$ в $(2.6)$ и, действительно, ведет к конечньм детерминантам. Мы опишем полубесконечные иерархии в настоящем пункте весьма коротко, оставляя некоторые технические вычисления для приложения 1 . Более подробное изложение может быть найдено в $[73,65]$.

Итак, мы будем искать точку грассманниана в виде

$$
G=G_{0} P_{+},
$$

где $P_{+}-$проектор на положительные состояния:

$$
P_{+}|n\rangle=\theta(n)|n\rangle
$$

Этот проектор можно естественно реализовать в терминах фермионов

$$
P_{+}=: \exp \left[\sum_{i<0} \psi_{i} \psi_{i}^{*}\right]:
$$

Он обладает рядом свойств:

$$
\begin{gathered}
P_{+} \psi_{-k}^{*}=\psi_{-k} P_{+}=0, \quad k>0 \\
{\left[P_{+}, \psi_{k}\right]=\left[P_{+}, \psi_{k}^{*}\right]=0, \quad k \geq 0} \\
P_{+}^{2}=P_{+}
\end{gathered}
$$


Если этот проектор вставлен в $(2.1)$, естественно потребовать, чтобы $G_{0}$ зависел только от $\psi_{k}$ и $\psi_{k}^{*}$ с $k \geq 0$. Мы выбираем его в виде

$$
G_{0}=: \exp \left\{\left(\int_{\gamma} A(z, w) \psi_{+}(z) \psi_{+}^{*}\left(w^{-1}\right) d z d w\right)-\sum_{i \geq 0} \psi_{i} \psi_{i}^{*}\right\}:,
$$

где $\gamma$ - некоторая область интегрирования. Ключевая формула, доказательство которой мы оставляем до приложения 1 , дает $\tau$-функцию с элементом грассманниана, выбранным в форме (2.47):

$$
\begin{aligned}
\tau_{n}(t, \bar{t}) & =\left\langle n\left|e^{H(t)} G_{0} P_{+} e^{\bar{H}(\bar{t})}\right| n\right\rangle= \\
& =\frac{1}{n !} \int_{\gamma} \Delta(w) \Delta(z) \prod_{i=1}^{n} A\left(z_{i}, w_{i}\right) e^{\xi\left(t, z_{i}\right)+\xi\left(\bar{t}, w_{i}\right)} d z_{i} d w_{i}
\end{aligned}
$$

где $\Delta(z) \equiv \operatorname{det}_{i, j} z_{j}^{i-1}$ - определитель Ван-дер-Монда.

Вычислениями, абсолютно аналогичными проделанным выше (см. также приложение 1), можно получить детерминантное представление для полубесконечной иерархии:

$$
\tau_{n}(t, \bar{t})=\left.\operatorname{det}\left[\partial_{t_{1}}^{i} \partial_{\bar{t}_{1}}^{j} \int_{\gamma} A(z, w) e^{\xi(t, z)+\xi(\bar{t}, w)} d z d w\right]\right|_{i, j=0, \ldots, n-1} .
$$

Это, действительно, детерминант конечной матрицы, в частных случаях даюший детерминантные представления соответствуюших матричных моделей [65].

Заметим, что матрица $A_{i j}$, определенная из разложения $A(z, w)$ по степеням $z$ и $w$, совпадает с матрицей $R_{i j}$ из (2.9) (см. приложение 1 ). Такая простая связь матрицы врашения фермионов с матрищей, задаюшей элемент грассманниана, является отличительной чертой полубесконечных иерархий.

Элемент грассманниана, определенный в (2.47), далеко не единственно возможный. Связано это с различными возможными способами продолжить соотношение (2.46) на отрицательные значения $n$. Принятый здесь способ отвечает условию

$$
\tau_{n}=0 \quad \text { при } \quad n<0 .
$$

Этот способ хорош тем, что при редукции к цепочке Тоды определенная таким образом $\tau$-функция зависит только от суммы времен $t_{k}+\bar{t}_{k}$, что является определяюшим свойством для соответствующей бесконечной иерархии. Другие интересные выборы элемента $G$ обсуждались в [73]. Если рассмотреть зависимость только от времен $t_{k}$, один из них отвечает, например, условию

$$
\tau_{n}\left(t_{k}\right)=\tau_{-n}\left((-)^{k} t_{k}\right)
$$

и описывает (модифицированную) симплектическую иерархию КП [59], т.е. соответствуюший элемент грассманниана принадлежит $S p(\infty)$. Другой выбор элемента грассманниана (просто $G=G_{0}$ ) приводит к условию

$$
\tau_{n}=1 \quad \text { при } \quad n<0
$$

и т.д. 


\section{3. ТЕОРЕТИКО-ГРУППОВЫЕ СТРУКТУРЫ МОЛЕКУЛЫ ТОДЫ}

Прежде чем переходить к обобщению построенной в предыдущем разделе конструкции, мы обсудим теоретико-групповые структуры, которые присутствуют в интегрируемых системах, в качестве основного примера рассматривая теорию Лиувилля (Тоды $)^{9)}$. Заметим, что в одной $u$ той же интегрируемой системе часто представлены разные групповые структуры. В частности, некоторые группы действуют в пространстве решений интегрируемой иерархии, другие - просто на пространство переменных интегрируемых уравнений (в “пространстве-времени”) [40]. Мы начнем с рассмотрения структур первого типа, в то время как вторые рассмотрены в разделе 4.

Как мы обсуждали в предыдушем разделе, все решения интегрируемой иерархии 2РТ (2.1) описываются групповыми элементами группы $G L(\infty)$ (2.6). Эта группа действует в пространстве решений и одновременно в пространстве спектрального параметра. Именно групповая структура этого типа будет нас в основном интересовать, поскольку как раз она ответственна за большинство определяюших свойств интегрируемой системы и непосредственно деформируется при квантовании системы. В примере, который мы сейчас рассмотрим - системе Лиувилля, присутствует, однако, еще одна группа.

Мы начнем с естественного ограничения полубесконечных иерархий, заданных условием (2.56), на конечные системы. Для этого мы наложим еще одно, дополнительное условие

$$
\tau_{n}=0, \quad n>p,
$$

для некоторого $p$. Эта система отвечает двумеризованной $(p-1)$-частичной молекуле Тоды [78-80] и иногда называется непериодической $S L(p)$-цепочкой Тоды [75]. Поскольку она является очень частным случаем полубесконечной иерархии, мы можем воспользоваться формулами предыдушего раздела и приложения 1. Тогда можно проверить, что $S L(p)$-молекула Тоды описьвается матрицей врашения фермионов $R_{i j}$, имеющей ранг $p$, а в остальном являющейся произвольной [36]. Это означает, что она может быть представлена в виде

$$
R_{i j}=\sum_{k}^{p} f_{i}^{(k)} g_{j}^{(k)}
$$

где $f_{i}^{(k)}$ и $g_{j}^{(k)}$ - произвольные коэффициенты. При этом ядро элемента грассманниана $A(z, w)(\Pi 1.8)$, разложение которого по степеням $z$ и $w$ дает матрицу $R_{i j}$, имеет вид

$$
A(z, w)=\sum_{k}^{p} f^{(k)}(z) g^{(k)}(w)
$$

где $f^{(k)}(z)$ и $g^{(k)}(z)$ - произвольные функции.

Проверить это утверждение проще всего, использовав детерминантное представление (П1.25). Действительно, следствием первого уравнения иерархии 2РТ

$$
\tau_{n} \partial_{t_{1}} \partial_{\bar{t}_{1}} \tau_{n}-\partial_{t_{1}} \tau_{n} \partial_{\bar{t}_{1}} \tau_{n}=\tau_{n+1} \tau_{n-1}
$$

\footnotetext{
9) В действительности уже весьма давно [75, 76] (см. также, по-видимому, самую первую работу на эту тему [77]) было обнаружено, что язык теории групп является одним из самых эффективных и элегантных подходов к описанию классических интегрируемых систем.
} 
и условия (3.1) является то, что $\log \tau_{0}$ и $\log \tau_{p}$ удовлетворяют волновому уравнению

$$
\partial_{t_{1}} \partial_{\bar{t}_{1}} \log \tau_{0}=\partial_{t_{1}} \partial_{\bar{t}_{1}} \log \tau_{p}=0
$$

Поскольку относительная нормировка $\tau_{n}$ не фиксирована, мы всегда можем выбрать $\tau_{0}=1$. Тогда

$$
\tau_{0}(t)=1, \quad \tau_{p}(t)=\chi\left(t_{1}\right) \bar{\chi}\left(\bar{t}_{1}\right),
$$

где $\chi\left(t_{1}\right)$ и $\bar{\chi}\left(\bar{t}_{1}\right)$ - произвольные функции. Двумеризованная модель Тоды с граничными условиями (3.6) была рассмотрена в $[78,79]$. Решение уравнения (3.4) в этом случае имеет вид [80]

$$
\tau_{n}(t)=\operatorname{det} \partial_{t_{1}}^{i-1}\left(-\partial_{\bar{t}_{1}}\right)^{j-1} \tau_{1}(t)
$$

C

$$
\tau_{1}(t)=\sum_{k=1}^{p} a^{(k)}(t) \bar{a}^{(k)}\left(\bar{t}_{1}\right),
$$

где функции $a^{(k)}(t)$ и $\bar{a}^{(k)}\left(\bar{t}_{1}\right)$ удовлетворяют условиям

$$
\operatorname{det} \partial_{t_{1}}^{i-1} a^{(k)}(t)=\chi(t), \quad \operatorname{det}\left(-\partial_{\bar{t}_{1}}\right)^{i-1} \bar{a}^{(k)}\left(\bar{t}_{1}\right)=\bar{\chi}\left(\bar{t}_{1}\right)
$$

Этот результат отвечает формуле (П1.25) с ядром $A(z, w)$ вида (3.3). Подчеркнем, что хотя молекула Тоды выглядит как полубесконечная иерархия с еще одним, дополнительным проектором, она описывается, вообше говоря, бесконечным числом фермионных мод, поскольку матрица (3.2) имеет бесконечное число ненулевых матричных элементов.

Заметим, что функция $\tau_{p}$ может быть положена равной 1 . Именно такой выбор отвечает группе $S L(p)$, обшее же решение - группе $G L(p)$.

Обсудим теперь, как можно увидеть эту групповую структуру в молекуле Тоды. Для простоты мы далее рассмотрим пример системы Лиувилля $(p=2)$, поскольку обобщение на старшие группы выполняется прямолинейно. Простейший и непосредственный способ увидеть эту групповую структуру - это восстановить явную групповую структуру в выражениях (3.7), (3.8), что и было сделано в [81, 82]. Более того, такоепредставление допускает деформацию, так что квантовой системе Лиувилля отвечает просто квантовая группа $S L_{q}(2)$ [83-86]. Тем не менее для наших целей здесь удобнее использовать несколько иной взгляд на проблему [36].

А именно, мы вернемся к рассмотрению теории Лиувилля как редукции из общей двумеризованной модели Тоды, отвечающей группе $G L(\infty)$. Тогда функции $f^{(k)}(z)$ и $g^{(k)}(z)$ (или набор соответствуюших коэффициентов $f_{i}^{(k)}$ и $g_{i}^{(k)}$ ) описывают способ, каким группа $S L(2)$, отвечаюшая системе Лиувилля, погружается при редукции в группу $G L(\infty)$. При этом различные погружения связаны внешними $G L(\infty)$-автоморфизмами группы $S L(2)$.

Опишем теперь это более явными выражениями. Уравнение Лиувилля имеет следующий вид:

$$
\partial \tau_{1} \bar{\partial} \tau_{1}-\tau_{1} \partial \bar{\partial} \tau_{1}=\tau_{0} \tau_{2}=1
$$

или

$$
\partial \bar{\partial} \phi=2 e^{\phi}, \quad \tau_{1}=e^{-\phi / 2}
$$


Его обшее решение

$$
\tau_{1}(t, \bar{t} \mid g)=(1+A(t) B(\bar{t}))\left[\frac{\partial A}{\partial t} \frac{\partial B}{\partial \bar{t}}\right]^{-\frac{1}{2}}
$$

параметризуется двумя произвольными функциями $A(t)$ и $B(\bar{t})$. Эти функции связаны с матрищей врашения фермионов следуюшими формулами, которые легко могут быть получены сравнением (П1.25) и (3.12):

$$
\begin{aligned}
G \psi_{i} G^{-1}= & \left(\iint d t \frac{e^{-t x}}{\sqrt{\partial A}} d x x^{i-1}\right) \cdot \sum_{k}\left(\iint d \bar{t} \frac{e^{-\bar{t} y}}{\sqrt{\bar{\partial} B}} d y y^{k-1}\right) \psi_{k}+ \\
& +\left(\iint d t \frac{e^{-t x} A}{\sqrt{\partial A}} d x x^{i-1}\right) \cdot \sum_{k}\left(\iint d \bar{t} \frac{e^{-\bar{t} y} B}{\sqrt{\bar{\partial} B}} d y y^{k-1}\right) \psi_{k} \equiv \\
\equiv & f_{i} \Psi^{(1)}+g_{i} \Psi^{(2)} .
\end{aligned}
$$

Таким образом, элемент $G$ грассманниана, отвечающий системе Лиувилля, врашает фермионные моды в двумерном пространстве, заданном “одетыми” фермионами $\Psi_{i}^{(1,2)}$, которые зависят, естественно, от конкретного выбора $G$, т.е. от функций $A$ и $B$. Каждый выбор $G$ фиксирует такую пару фермионов, в свою очередь, задающих погружение группы $S L(2)$ в группу $G L(\infty)$. При этом все многообразие решений уравнения Лиувилля соответствует всем возможным выборам функций $A$ и $B$, т.е. всем возможным подобным погружениям.

\section{4. ОБОБЩЕННАЯ $\tau$-ФУНКЦИЯ И БИЛИНЕЙНЫЕ ТОЖДЕСТВА}

В нашем предыдущем обсуждении $\tau$-функций мы в основном сосредоточили внимание на их детерминантных представлениях. $\tau$-Функцию, однако, можно ввести и иерархией определяющих уравнений - (бесконечным) набором билинейных уравнений Хироты типа (3.4) и (2.45). Далее мы покажем, как обобщить понятие $\tau$-функции так, чтобы она по-прежнему удовлетворяла иерархии билинейных тождеств, а в следуюшем разделе обсудим ее детерминантные представления, которые оказываются более частным свойством.

Основная идея нашего подхода - полностью алгебраизовать интегрируемую иерархию, так чтобы ее можно было построить по любому представлению (старшего веса) любой группы. В том числе, квантование отвечает замене группы на ее квантовый аналог.

\section{1. $\tau$-Функция и билинейные тождества}

$\tau$-Функция. Итак, для данной универсальной обертываюшей алгебры (УОА $U(\mathcal{G})$ (над некоторым кольцом) и модуля Верма $V$ этой алгебры мы определяем $\tau$-функцию как производящую функцию всех матричных элементов $\langle m|g| \bar{m}\rangle_{V}[35]$ :

$$
\tau_{V}(t, \bar{t} \mid g) \equiv \sum_{m, \bar{m} \in V} s_{m, \bar{m}}^{V}(t, \bar{t})\langle m|g| \bar{m}\rangle_{V}
$$


Основная неоднозначность в этом определении связана с выбором функции $s_{m, \bar{m}}^{V}(t, \bar{t})$, который необходимо сделать некоторым “правильным" образом, чтобы получить $\tau$-функцию с хорошими свойствами. Эта неоднозначность частично убирается для представлений старшего веса, когда можно потребовать, чтобы выполнялось

$$
\tau_{V}(t, \bar{t} \mid g)=\left\langle 0_{V}|U(t) g \bar{U}(\bar{t})| 0_{V}\right\rangle
$$

с некоторыми операторами $U$ и $\bar{U}$, не зависяшими от $V$, где $\left|0_{V}\right\rangle$ - вектор старшего ве$\mathrm{ca}^{10)}$.

Это требование естественно возникает из рассмотрения $\tau$-функции иерархии $2 \mathrm{PT}$, которая отвечает $\mathbf{G}=S L(p)$ с $V$ - одним из $p-1$ фундаментальных представлений. Мы вернемся к этому случаю подробнее в разделе 5 .

Однако набор билинейных соотношений, которые мы теперь собираемся вывести, не зависит от конкретного выбора функций $s_{m, \bar{m}}^{V}(t, \bar{t})$, поэтому мы вернемся к их явному виду позднее.

Сплетающие операторы и билинейные тождества. Обобщим теперь вывод БТ, продемонстрированный в разделе 2 , на случай обшей $\tau$-функции (4.1). Его полностью алгебраизуемой частью является получение соотношения, аналогичного (2.39). При этом сама процедура вывода теперь состоит из нескольких шагов.

1. Стартовой точкой является погружение модуля Верма $\widehat{V}$ в тензорное произведение $V \otimes W$, где $W$ - некоторое (произвольное) конечномерное представление $\mathcal{G}^{11)}$. При фиксированном выборе $V$ и $W$ сушествует только конечное число возможностей для $\widehat{V}$.

Определим теперь аналоги фермионов - сплетаюшие операторы. Так, правый сплетающий оператор типа $W$ определен как гомоморфизм $\mathcal{G}$-модулей:

$$
E_{R}: \widehat{V} \rightarrow V \otimes W
$$

Этот сплетающий оператор может быть построен для представления старшего веса

$$
|\mathbf{0}\rangle_{\widehat{V}}=\left(\sum_{\left\{p_{\alpha}, i_{\alpha}\right\}} A\left\{p_{\alpha}, i_{\alpha}\right\}\left(\prod_{\alpha>0}\left(T_{-\alpha}\right)^{p_{\alpha}} \otimes\left(T_{-\alpha}\right)^{i_{\alpha}}\right)\right)|\mathbf{0}\rangle_{V} \otimes|\mathbf{0}\rangle_{W}
$$

а затем явно продолжен на все представление по формуле

$$
\widehat{V}=\left\{\left|\mathbf{n}_{\alpha}\right\rangle_{\widehat{V}}=\prod_{\alpha>0}\left(\Delta\left(T_{-\alpha}\right)^{n_{\alpha}}|\mathbf{0}\rangle_{\widehat{V}}\right\}\right.
$$

\footnotetext{
10) Эволюционные операторы $U(t)(\bar{U}(\bar{t}))$ для общего представления зависят от всех повышающих (понижающих) генераторов алгебры, которые, вообще говоря, не коммутируют. Поэтому потоки по временам в описываемых ниже уравнениях иерархии не должны коммутировать, в отличие от случая стандартных интегрируемых иерархий. При специальных выборах представлений, например в фундаментальных представлениях классических групп, эти эволюционные операторы тем не менее могут коммутировать - см. подробнее раздел 6 .

11) В случае афффинных алгебр необходимо использовать также конечномерные (evaluation) представления, это представления с нулевым центральным зарядом, но они не являются представлениями старшего веса (ср. с определением вершинных операторов в $[87,88])$.
} 
где $T_{-\alpha}$ - (понижающие) генераторы алгебры, лежащие в ее максимальной отрицательной (правой) нильпотентной подалгебре $\bar{N}(\mathcal{G}), \alpha$ - положительные корни, вакуумное состояние аннигилируется всеми повышающими операторами $T_{\alpha}$, лежащими в положительной (левой) нильпотентной подалгебре $N(\mathcal{G})$, а модуль Верма строится действием всех генераторов $T_{-\alpha}$ на состояние старшего веса: $V=\left\{\left|\mathbf{n}_{\alpha}\right\rangle_{V}=\prod_{\alpha>0}\left(T_{-\alpha}\right)^{n_{\alpha}}|\mathbf{0}\rangle_{V}\right\}$. Формула (4.5) выражает тот факт, что действие $\mathcal{G}$ на тензорном произведении представлений (модулей Верма) определяется копроизведением $\Delta$ и для конечномерных $W$ позволяет представить каждое $\left|\mathbf{n}_{\alpha}\right\rangle_{\widehat{V}}$ в виде конечной суммы состояний $\left|\mathbf{m}_{\alpha}\right\rangle_{V}$ с коэффициентами, принимающими значение в элементах $W$.

2. На следующем шаге рассмотрим другую тройку модулей, определяющих левый сплетающий оператор,

$$
E_{L}^{\prime}: \widehat{V}^{\prime} \rightarrow W^{\prime} \otimes V^{\prime}
$$

такую, что произведение $W \otimes W^{\prime}$ содержит единичное представление $\mathcal{G}$.

БТ в терминах УОА. Теперь мы переходим непосредственно к выводу БТ. Они могут быть получены в двух формах - в операторной, аналогичной формуле (2.38), и в виде соотношения на матричные элементы (т.е. в терминах алгебры функций на группе). Первую форму можно получить в два шага. На первом шаге нужно рассмотреть проекцию произведения $W \otimes W^{\prime}$ на единичное представление

$$
\pi: W \otimes W^{\prime} \rightarrow I
$$

явно обеспечиваемую умножением любого элемента из $W \otimes W^{\prime}$ на

$$
\pi={ }_{W}\langle\mathbf{0}| \otimes{ }_{W^{\prime}}\langle\mathbf{0}|\left(\sum_{\left\{i_{\alpha}, i_{\alpha}^{\prime}\right\}} A_{\pi}\left\{i_{\alpha}, i_{\alpha}^{\prime}\right\}\left(\prod_{\alpha>0}\left(T_{+\alpha}\right)^{i_{\alpha}} \otimes\left(T_{+\alpha}\right)^{i_{\alpha}^{\prime}}\right)\right) .
$$

Используя эту проекцию, можно построить новый сплетающий оператор

$$
\Gamma: \widehat{V} \otimes \widehat{V}^{\prime} \stackrel{E_{R} \otimes E^{\prime}}{\longrightarrow} V \otimes W \otimes W^{\prime} \otimes V^{\prime} \stackrel{I \otimes \pi \otimes I}{\longrightarrow} V \otimes V^{\prime}
$$

которьй обладает свойством (2.38):

$$
\Gamma(g \otimes g)=(g \otimes g) \Gamma
$$

для любого группового элемента $g$ такого, что

$$
\Delta(g)=g \otimes g
$$

Иными словами, пространство $W \otimes W^{\prime}$ содержит канонический элемент спаривания $w_{i} \otimes w^{i}$, которьй коммутирует с действием $\Delta(g)$. Это означает, что оператор $\sum_{i} E_{i} \otimes$ $E^{i}: V \otimes V^{\prime} \rightarrow \widehat{V} \otimes \widehat{V}^{\prime}\left(E_{i} \equiv E\left(w_{i}\right), E^{i} \equiv E\left(w^{i}\right)\right)$ коммутирует с $\Delta(g)$.

Тождество (4.10) является алгебраической формулировкой БТ. Для получения дифференциальных (или разностных) тождеств теперь, как и в рассмотренном выше примере иерархии 2РТ, мы должны использовать определение (4.2) и рассмотреть матричный элемент тождества (4.10) вместе с эволюционными операторами между состояниями старшего веса. Это приводит к тождествам (сушествует множество эквивалентных БТ 
в соответствии со многими возможными выборами представлений $V$ и $\widehat{V}$ ) на объекты типа ФБА, т.е. на средние (4.2) с дополнительными вставками сплетаюших операторов $E_{i}$ и $E^{i}$. Далее, используя коммутационные соотношения сплетаюших операторов с генераторами алгебры, можно пронести $E_{i}$ до вектора старшего веса. Результат этого коммутирования может быть смоделирован действием некоторых дифференциальных или разностных операторов, что и приводит к дифференциальным или разностным БТ. Этот последний шаг, однако, не всегда возможен, он требует правильного выбора операторов эволюции $U(t), \bar{U}(\bar{t})$. До сих пор не сушествует общего рецепта для такого выбора. Далее, однако, мы построим несколько явных примеров, иллюстрируюших, как этот выбор обычно делается. Конечно, дифференциальная (разностная) форма БТ сильно зависит от конкретной алгебры и выбранного представления и не может быть получена в общей алгебраической форме. Более того, как мы покажем далее, для одной и той же системы можно получить разные дифференциальные иерархии или даже дифференциальные и разностные иерархии в зависимости от выбора операторов эволюции.

БТ в терминах алгебры функций. Ниже мы получим БТ в терминах матричных элементов, т.е. в терминах алгебры функций на группе. Этот язык дуален языку УОА.

А именно, напишем матричный элемент (4.10) между четырьмя состояниями

$$
{ }_{V^{\prime}}\left\langle\left. k^{\prime}\right|_{V}\langle k|(g \otimes g) \Gamma| n\rangle_{\widehat{V}} \mid n^{\prime}\right\rangle_{\widehat{V}^{\prime}}={ }_{V^{\prime}}\left\langle\left. k^{\prime}\right|_{V}\langle k|\Gamma(g \otimes g)| n\rangle_{\widehat{V}} \mid n^{\prime}\right\rangle_{\hat{V}^{\prime}} .
$$

Действие оператора Г может быть представлено формулой

$$
\Gamma|n\rangle_{\hat{\lambda}}\left|n^{\prime}\right\rangle_{\hat{\lambda}^{\prime}}=\sum_{l, l^{\prime}}|l\rangle_{\lambda}\left|l^{\prime}\right\rangle_{\lambda^{\prime}} \Gamma\left(l, l^{\prime} \mid n, n^{\prime}\right),
$$

т.е. (4.12) преврашается в

$$
\begin{aligned}
& \sum_{m, m^{\prime}} \Gamma\left(k, k^{\prime} \mid m, m^{\prime}\right) \frac{\|k\|_{\lambda^{\prime}}^{2}\left\|k^{\prime}\right\|_{\lambda^{\prime}}^{2}}{\|m\|_{\hat{\lambda}^{2}}^{2}\left\|m^{\prime}\right\|_{\hat{\lambda}^{\prime}}^{2}}\langle m|g| n\rangle_{\hat{\lambda}}\left\langle m^{\prime}|g| n^{\prime}\right\rangle_{\hat{\lambda}^{\prime}}= \\
& \quad=\sum_{l, l^{\prime}}\langle k|g| l\rangle_{\lambda}\left\langle k^{\prime}|g| l^{\prime}\right\rangle_{\lambda^{\prime}} \Gamma\left(l, l^{\prime} \mid n, n^{\prime}\right) .
\end{aligned}
$$

Для того чтобы переписать это выражение как дифференциальное или разностное уравнение, нужно использовать формулу (4.1) для $\tau$-функции. Тогда можно выписать производящее уравнение для тождеств (4.14), пользуясь явными формулами для матричных элементов $\Gamma\left(l, l^{\prime} \mid n, n^{\prime}\right)$, которые могут быть вычислены в рамках теории групा (это просто соответствуюшие коэффициенты Клебша-Гордана). Опять для представления этого производяшего уравнения в дифференциальной (разностной) форме требуется правильный выбор коэффициентов $s_{m, \bar{m}}^{R}(t, \bar{t})$ в формуле (4.1). Технически этот выбор часто проше проделать именно в данном представлении, т.е. для БТ, сформулированных в терминах алгебры функций. Мы проиллюстрируем этот метод в следующем разделе.

В заключение данного пункта заметим, что если бы мы хотели буквально воспроизвести вывод БТ, который был проделан для иерархии 2 РТ, нам нужно было бы для $о д-$ ной и той же тройки представлений $V, V^{\prime}, W$ ввести сопряженную пару сплетающих операторов

$$
\Phi: \widehat{V} \otimes W \rightarrow V, \quad \Phi^{\star}: V \rightarrow W \otimes \widehat{V},
$$


которые по определению удовлетворяют условиям

$$
\Delta(g) \Phi=\Phi g, \quad \Phi^{\star} \Delta(g)=g \Phi^{\star},
$$

т.к. являются гомоморфизмами, а действие алгебры в тензорном произведении задается коумножением. Эти условия являются буквальным обобщением формулы (2.9), а канонический элемент опять коммутирует с групповым элементом $g$, давая уравнение (4.10). Однако предложенный нами вьвод БТ с двумя тройками представлений кажется более общим, поэтому на протяжении настоящего раздела мы будем использовать именно его.

\section{2. Пример. Квантовая группа $S L_{q}(2)$}

Рассмотрим теперь в качестве примера, иллюстрируюшего приведенную выше несколько абстрактную конструкцию обобщенной $\tau$-функции и БТ, случай квантовой группы $S L_{q}(2)$ [35]. Одновременно этот пример служит иллюстрацией того, как устроены $\tau$-функция и БТ для квантовых интегрируемых систем. Как уже говорилось, в рамках описываемого подхода квантование отвечает просто замене группы ее квантовым аналогом. Заметим, что здесь и далее, говоря о $q$-деформациях, мы всегда подразумеваем вешественный параметр $q>1$, хотя остальные значения $q$ могут также легко быть рассмотрены с помощью развитых методов.

Билинейные тождества. Алгебра $U_{q}(S L(2))$ задается генераторами $T_{+}, T_{-}$и $T_{0}$ с коммутационными соотношениями ${ }^{12}$ )

$$
q^{T_{0}} T_{ \pm} q^{-T_{0}}=q^{ \pm 1} T_{ \pm}, \quad\left[T_{+}, T_{-}\right]=\frac{q^{2 T_{0}}-q^{-2 T_{0}}}{q-q^{-1}}
$$

и коумножением

$$
\Delta\left(T_{ \pm}\right)=q^{T_{0}} \otimes T_{ \pm}+T_{ \pm} \otimes q^{-T_{0}}, \quad \Delta\left(q^{T_{0}}\right)=q^{T_{0}} \otimes q^{T_{0}} .
$$

Модуль Верма $V_{\lambda}$ старшего веса $\lambda$ (не обязательно полуцелого) состоит из элементов

$$
|n\rangle_{\lambda} \equiv T_{-}^{n}|0\rangle_{\lambda}, \quad n \geq 0,
$$

таких, что

$$
\begin{gathered}
T_{-}|n\rangle_{\lambda}=|n+1\rangle_{\lambda}, \quad T_{0}|n\rangle_{\lambda}=(\lambda-n)|n\rangle_{\lambda}, \quad T_{+}|n\rangle_{\lambda} \equiv b_{n}(\lambda)|n-1\rangle_{\lambda} \\
b_{n}(\lambda)=[n]_{q}[2 \lambda+1-n]_{q}, \quad[x]_{q} \equiv \frac{q^{x}-q^{-x}}{q-q^{-1}}, \quad[n]_{q} ! \equiv[1]_{q}[2]_{q} \ldots[n]_{q} \\
\|n\|_{\lambda}^{2} \equiv{ }_{\lambda}\langle n \mid n\rangle_{\lambda}=\frac{[n]_{q} ! \Gamma_{q}(2 \lambda+1)}{\Gamma_{q}(2 \lambda+1-n)} \stackrel{\lambda \in \mathbf{Z} / 2}{=} \frac{[2 \lambda]_{q} ![n]_{q} !}{[2 \lambda-n]_{q} !}
\end{gathered}
$$

Теперь мы получим БТ, работая в терминах алгебры функций на квантовой группе, т.е. явно вычисляя матричные элементы оператора Г.

В качестве модуля $W$ мы выбираем неприводимое представление $U_{q}(S L(2))$ со спином $1 / 2$, тогда $\widehat{V}=V_{\lambda \pm 1 / 2}, V=V_{\lambda}$.

${ }^{12)}$ Все необходимые факты о квантовых группах, используемые ниже, могут быть найдены в обзоре [89]. 
Для вычисления матричных элементов Г нужно спроецировать тензорное произведение двух разных $W$ на синглетное состояние $S=|+\rangle|-\rangle-q|-\rangle|+\rangle$ :

$$
(A|+\rangle+B|-\rangle) \otimes(|+\rangle C+|-\rangle D) \rightarrow A D-q B C .
$$

При нашем выборе $W$ нужно рассмотреть два различных случая:

случай А: $\widehat{V}=V_{\lambda-\frac{1}{2}} \quad$ и $\quad \widehat{V}^{\prime}=V_{\lambda^{\prime}-\frac{1}{2}}$ или

случай В: $\widehat{V}=V_{\lambda-\frac{1}{2}} \quad$ и $\quad \widehat{V}^{\prime}=V_{\lambda^{\prime}+\frac{1}{2}}$.

Пользуясь формулами (4.5) и (4.18), теперь нетрудно вычислить матричные элементы оператора проецирования ${ }^{13)}$.

Случай А:

$$
\begin{aligned}
|n\rangle_{\lambda-\frac{1}{2}}\left|n^{\prime}\right\rangle_{\lambda^{\prime}-\frac{1}{2}} \rightarrow & q^{\frac{n^{\prime}-n-1}{2}}\left(\left[n^{\prime}-2 \lambda^{\prime}\right]_{q} q^{\lambda^{\prime}}|n+1\rangle_{\lambda}\left|n^{\prime}\right\rangle_{\lambda^{\prime}-}\right. \\
& \left.-[n-2 \lambda]_{q} q^{-\lambda}|n\rangle_{\lambda}\left|n^{\prime}+1\right\rangle_{\lambda^{\prime}}\right) .
\end{aligned}
$$

Случай B:

$$
|n\rangle_{\lambda+\frac{1}{2}}\left|n^{\prime}\right\rangle_{\lambda^{\prime}-\frac{1}{2}} \rightarrow q^{\frac{n^{\prime}-n-1}{2}}\left(\left[n^{\prime}-2 \lambda^{\prime}\right]_{q} q^{\lambda^{\prime}}|n\rangle_{\lambda}\left|n^{\prime}\right\rangle_{\lambda^{\prime}}-[n]_{q} q^{+\lambda+1}|n-1\rangle_{\lambda}\left|n^{\prime}+1\right\rangle_{\lambda^{\prime}}\right) .
$$

Используя явные формулы (4.22), (4.23) для матричных элементов $\Gamma\left(l, l^{\prime} \mid n, n^{\prime}\right)$, мы замечаем, что для тождеств (4.14) имеется простое производяшее уравнение (см. конец предыдущего пункта), если выбрать в качестве эволюционных операторов $q$-экспоненты $^{14)}$ от генераторов $U(t)=e_{q}\left(t T_{+}\right), \bar{U}(\bar{t})=e_{q}\left(\bar{t} T_{-}\right)$. Это производящее уравнение имеет вид:

Случай А:

$$
\begin{aligned}
& \sqrt{M_{\bar{t}}^{-} M_{\bar{t}^{\prime}}^{+}}\left(q^{\lambda^{\prime}} D_{\bar{t}}^{(0)} \bar{t}^{\prime} D_{\bar{t}^{\prime}}^{\left(2 \lambda^{\prime}\right)}-q^{-\lambda} \bar{t} D_{\bar{t}}^{(2 \lambda)} D_{\bar{t}^{\prime}}^{(0)}\right) \tau_{\lambda}(t, \bar{t} \mid g) \tau_{\lambda^{\prime}}\left(t^{\prime}, \bar{t}^{\prime} \mid g\right)= \\
& \quad=[2 \lambda]_{q}\left[2 \lambda^{\prime}\right]_{q} \sqrt{M_{t}^{-} M_{t^{\prime}}^{+}}\left(q^{-\left(\lambda+\frac{1}{2}\right)} t^{\prime}-q^{\left(\lambda^{\prime}+\frac{1}{2}\right)} t\right) \tau_{\lambda-\frac{1}{2}}(t, \bar{t} \mid g) \tau_{\lambda^{\prime}-\frac{1}{2}}\left(t^{\prime}, \bar{t}^{\prime} \mid g\right),
\end{aligned}
$$

здесь

$$
D_{t}^{(\alpha)} \equiv \frac{q^{-\alpha} M_{t}^{+}-q^{\alpha} M_{t}^{-}}{\left(q-q^{-1}\right) t}
$$

а $M^{ \pm}$- мультипликативные операторы сдвига: $M_{t}^{ \pm} f(t)=f\left(q^{ \pm 1} t\right)$.

Случай В:

$$
\begin{aligned}
& \sqrt{M_{\bar{t}}^{-} M_{\bar{t}^{\prime}}^{+}}\left(q^{\lambda^{\prime}} \bar{t}^{\prime} D_{\bar{t}^{\prime}}^{\left(2 \lambda^{\prime}\right)}-q^{(\lambda+1)} \bar{t} D_{\bar{t}^{\prime}}^{(0)}\right) \tau_{\lambda}(t, \bar{t} \mid g) \tau_{\lambda^{\prime}}\left(t^{\prime}, \bar{t}^{\prime} \mid g\right)= \\
& =\frac{\left[2 \lambda^{\prime}\right]_{q}}{[2 \lambda+1]_{q}} \sqrt{M_{t}^{-} M_{t^{\prime}}^{+}}\left(q^{\lambda^{\prime}} t D_{t}^{(2 \lambda+1)}-q^{\lambda} t^{\prime} D_{t}^{(0)}\right) \tau_{\lambda+\frac{1}{2}}(t, \bar{t} \mid g) \tau_{\lambda^{\prime}-\frac{1}{2}}\left(t^{\prime}, \bar{t}^{\prime} \mid g\right) .
\end{aligned}
$$

Классический предел этих уравнений имеет вид:

\footnotetext{
13) Чтобы упростить формулы, здесь и далее мы опускаем знак тензорного произведения из обозначения состояний $|+\rangle \otimes|0\rangle_{\lambda}$ и т.д.

14) $e_{q}(x) \equiv \sum_{n \geq 0} \frac{x^{n}}{[n]_{q} !}$.
} 
Случай А:

$$
\begin{gathered}
\left(2 \lambda \frac{\partial}{\partial \bar{t}^{\prime}}-2 \lambda^{\prime} \frac{\partial}{\partial \bar{t}}+\left(\bar{t}^{\prime}-\bar{t}\right) \frac{\partial^{2}}{\partial \bar{t} \partial \bar{t}^{\prime}}\right) \tau_{\lambda}(t, \bar{t} \mid g) \tau_{\lambda^{\prime}}\left(t^{\prime}, \bar{t}^{\prime} \mid g\right)= \\
=4 \lambda \lambda^{\prime}\left(t^{\prime}-t\right) \tau_{\lambda-\frac{1}{2}}(t, \bar{t} \mid g) \tau_{\lambda^{\prime}-\frac{1}{2}}\left(t^{\prime}, \bar{t}^{\prime} \mid g\right) .
\end{gathered}
$$

Случай В:

$$
\begin{aligned}
& {\left[\left(\bar{t}^{\prime}-\bar{t}\right) \frac{\partial}{\partial \bar{t}^{\prime}}-2 \lambda^{\prime}\right] \tau_{\lambda}(t, \bar{t} \mid g) \tau_{\lambda^{\prime}}\left(t^{\prime}, \bar{t}^{\prime} \mid g\right)=} \\
& \quad=\frac{2 \lambda^{\prime}}{2 \lambda+1}\left[\left(t-t^{\prime}\right) \frac{\partial}{\partial t}-2 \lambda-1\right] \tau_{\lambda+\frac{1}{2}}(t, \bar{t} \mid g) \tau_{\lambda^{\prime}-\frac{1}{2}}\left(t^{\prime}, \bar{t}^{\prime} \mid g\right) .
\end{aligned}
$$

Таким образом, мы получаем разные БТ, которым должна удовлетворять одна и та же $\tau$-функция. В действительности достаточно использовать, например, первое уравнение (случай А), для того чтобы полностью найти $\tau$-функцию.

Классический предел. Перед тем как двигаться дальше, рассмотрим случай классической группы $S L(2), \tau$-функция которой удовлетворяет БТ (4.26), (4.27). Можно построить общее решение этих уравнений, однако еше проше вычислить $\tau$-функцию прямо из определения (4.2). Для представления с произвольным спином $\lambda$ результат имеет вид

$$
\tau_{\lambda}={ }_{\lambda}\left\langle 0\left|e^{t T_{-}} g e^{\bar{t} T_{+}}\right| 0\right\rangle_{\lambda}=(a+b \bar{t}+c t+d t \bar{t})^{2 \lambda},
$$

где групповой элемент $g$ параметризован тремя параметрами:

$$
g=e^{x_{+} T_{+}} e^{x_{0} T_{0}} e^{x_{-} T_{-}}
$$

И

$$
a \equiv e^{\frac{1}{2} x_{0}}+x_{+} x_{-} e^{-\frac{1}{2} x_{0}}, \quad b \equiv x_{+} e^{-\frac{1}{2} x_{0}}, \quad c \equiv e^{-\frac{1}{2} x_{0}} x_{-}, \quad d \equiv e^{-\frac{1}{2} x_{0}},
$$

т.е.

$$
a d-b c=1 .
$$

Вернемся теперь к БТ. Как мы уже отмечали, достаточно изучить уравнение (4.26), поскольку каждое решение этого уравнения удовлетворяет всем остальным БТ, например уравнению (4.27) или другим, полученным при других выборах $V, \widehat{V}$ и $W$. Общее решение (4.26) является 3-параметрическим и, конечно, совпадает с (4.28).

Заметим, что (4.28) при $\lambda=1 / 2$ (фундаментальное представление) имеет мало общего с решением уравнения Лиувилля (3.12), которое, напомним, ассоциируется с $S L(2)$ редукцией (см. раздел 3$)$. Связь между ними, однако, имеется, хотя набор решений уравнения Лиувилля (3.10) гораздо богаче (решения (4.26) в этом наборе содержатся).

Выясним, как ограничивается набор решений уравнения Лиувилля. Для этого перепишем уравнение (4.26), как это обычно делается в уравнениях Хироты, в виде системы 
дифференциальных уравнений, полученных разложением по степеням $\epsilon=\left(t-t^{\prime}\right) / 2$ и $\bar{\epsilon}=\left(\bar{t}-\bar{t}^{\prime}\right) / 2$. К примеру, для $\lambda=\lambda^{\prime}$ получим из (4.26):

$$
\begin{array}{cl}
\text { коэфффициент перед } \epsilon: & \partial \tau_{\lambda} \bar{\partial} \tau_{\lambda}-\tau_{\lambda} \partial \bar{\partial} \tau_{\lambda}=2 \lambda \tau_{\lambda-\frac{1}{2}}^{2}, \\
\text { коэффиициент перед } \bar{\epsilon}: & 2 \lambda \tau_{\lambda} \bar{\partial}^{2} \tau_{\lambda}=(2 \lambda-1)\left(\bar{\partial} \tau_{\lambda}\right)^{2} .
\end{array}
$$

Если $\lambda=1 / 2$, первое из этих уравнений - просто уравнение Лиувилля $(3.10)$ :

$$
\partial \tau_{\frac{1}{2}} \bar{\partial} \tau_{\frac{1}{2}}-\tau_{\frac{1}{2}} \partial \bar{\partial} \tau_{\frac{1}{2}}=\tau_{0}^{2}=1
$$

в то время как второе

$$
\bar{\partial}^{2} \tau_{\frac{1}{2}}=0
$$

- условие, сильно ограничиваюшее вид возможных решений уравнения Лиувилля. Оно подразумевает, что две произвольные функции $A(t)$ и $B(\bar{t})$, параметризующие эти решения, сводятся к линейным функциям времен. На языке грассманниана все это означает, что рассматриваемая здесь $S L(2)$ - $\tau$-функция отвечает погружению матрищы $S L(2)$ в левый верхний угол матришы $G L(\infty)$ (см. раздел 3 ), чего, конечно, следовало ожидать, поскольку $S L(2)-\tau$-функция ничего "не знает" про группу $G L(\infty)$. В частности, для квантования "полноценного" уравнения Лиувилля необходимо вначале изучить систему $G L_{q}(\infty)$, а затем ее соответствуюшую редукцию.

Решения квантовых БТ. Перейдем теперь к решению квантовых БТ. Общее $c$-числовое решение уравнения (4.24) может быть явно построено. Действительно, легко проверить, что

$$
\tau_{\lambda}=\left[\alpha+\frac{1}{\alpha} t \bar{t}\right]_{q}^{2 \lambda} \equiv \sum_{i \geq 0} \frac{\Gamma_{q}(2 \lambda+1)}{\Gamma_{q}(2 \lambda+1-i)} \frac{\alpha^{2 \lambda-2 i}(t \bar{t})^{i}}{[i]_{q} !}
$$

удовлетворяет уравнению (4.24), поскольку

$$
\begin{aligned}
D_{t}^{(0)}\left[\alpha+\frac{1}{\alpha} t \bar{t}\right]_{q}^{2 \lambda} & =\frac{1}{\alpha}[2 \lambda]_{q}\left[\alpha+\frac{1}{\alpha} t \bar{t}\right]_{q}^{2 \lambda-1} \bar{t} \\
t D_{t}^{(2 \lambda)}\left[\alpha+\frac{1}{\alpha} t \bar{t}\right]_{q}^{2 \lambda} & =-\alpha[2 \lambda]_{q}\left[\alpha+\frac{1}{\alpha} t \bar{t}\right]_{q}^{2 \lambda-1}
\end{aligned}
$$

Это, однако, дает только однопараметрическое семейство решений в противоположность классическому случаю. Это объясняется тем, что из всех элементов $U_{q}(S L(2))$ только картановский имеет правильный закон коумножения (4.11), в то время как в классическом случае существует трехпараметрическое семейство групповых элементов (4.29), удовлетворяющих этому условию. 
Квантовая $\tau$-функция. Способ построения трехпараметрического семейства решений БТ, однако, имеется и в квантовом случае. Для этого достаточно рассмотреть некоммутативную $\tau$-функцию ${ }^{15)}$. Действительно, $(4.1)$ подразумевает, что $\tau$-функция принимает значения в алгебре функций на квантовой групше $S L_{q}(2)$, т.е. является некоммутативной величиной. Например, в фундаментальном представлении она равна

$$
\tau_{\frac{1}{2}}=\langle+|g|+\rangle+\bar{t}\langle+|g|-\rangle+t\langle-|g|+\rangle+t \bar{t}\langle-|g|-\rangle=a+b \bar{t}+c t+d t \bar{t}
$$

где генераторы $a, b, c, d$ алгебры функций $A\left(S L_{q}(2)\right)$ являются элементами матрицы

$$
\mathcal{T}=\left(\begin{array}{ll}
a & b \\
c & d
\end{array}\right), \quad a d-q b c=1
$$

с коммутационными соотношениями, заданными уравнениями $\mathcal{T} \mathcal{T} \mathcal{R}=\mathcal{R} \mathcal{T}[26]$ :

$$
\begin{gathered}
a b=q b a, \quad a c=q c a, \quad b d=q d b, \\
c d=q d c, \quad b c=c b, \quad a d-d a=\left(q-q^{-1}\right) b c .
\end{gathered}
$$

Для того чтобы получить такую некоммутативную $\tau$-функцию из выражения (4.2), нужно рассмотреть $g$ как элемент УОА, заданной над некоторым некоммутативным кольцом вместо поля комплексных чисел. Это увеличит число групповых элементов, удовлетворяюших условию (4.11). Таким кольцом является в точности $A_{q}(S L(2))$ (см. следующий пункт).

Для того чтобы сконструировать некоммутативную $\tau$-функцию в любом представлении спина $\lambda$, можно разложить это представление по представлениям со спинами $\lambda-1 / 2$ и $1 / 2[35]$ :

$$
\begin{aligned}
{ }_{\lambda}\langle k|g| n\rangle_{\lambda}= & q^{-\frac{k+n}{2}}\left[{ }_{\lambda-\frac{1}{2}}\langle k|g| n\rangle_{\lambda-\frac{1}{2}}\langle+|g|+\rangle+q^{\lambda}[n]_{q \lambda-\frac{1}{2}}\langle k|g| n-1\rangle_{\lambda-\frac{1}{2}}\langle+|g|-\rangle+\right. \\
& +q^{\lambda}[k]_{q \lambda-\frac{1}{2}}\langle k-1|g| n\rangle_{\lambda-\frac{1}{2}}\langle-|g|+\rangle+ \\
& \left.+q^{2 \lambda}[k]_{q}[n]_{q-\frac{1}{2}}\langle k-1|g| n-1\rangle_{\lambda-\frac{1}{2}}\langle-|g|-\rangle\right]_{q}
\end{aligned}
$$

При $\lambda \in \mathbf{Z} / 2$ рекуррентное применение этой процедуры дает

$$
\begin{aligned}
\tau_{\lambda}(t, \bar{t} \mid g)= & \tau_{\lambda-\frac{1}{2}}\left(q^{-\frac{1}{2}} t, q^{-\frac{1}{2}} \bar{t} \mid g\right) \tau_{\frac{1}{2}}\left(q^{\lambda-\frac{1}{2}} t, q^{\lambda-\frac{1}{2}} \bar{t} \mid g\right)= \\
& =\tau_{\frac{1}{2}}\left(q^{\frac{1}{2}-\lambda} t, q^{\frac{1}{2}-\lambda} \bar{t} \mid g\right) \tau_{\frac{1}{2}}\left(q^{\frac{3}{2}-\lambda} t, q^{\frac{3}{2}-\lambda} \bar{t} \mid g\right) \ldots \tau_{\frac{1}{2}}\left(q^{\lambda-\frac{1}{2}} t, q^{\lambda-\frac{1}{2}} \bar{t} \mid g\right) .
\end{aligned}
$$

\footnotetext{
${ }^{15)}$ Идея рассмотрения некоммутативной $\tau$-функции была также предложена в [86].
} 


\section{3. Универсальный Т-оператор (групповой элемент)}

Общая конструкция. Опишем теперь более явно конструкцию группового элемента, заданного над некоммутативным кольцом - алгеброй функций на квантовой группе, а именно, мы построим такой элемент $g \in U_{q}(\mathcal{G}) \otimes A(\mathbf{G})$ тензорного произведения УОА $U_{q}(\mathcal{G})$ и дуальной ей алгебры функций $A(\mathbf{G})$, что

$$
\Delta_{U}(g)=g \otimes_{U} g \in A(\mathbf{G}) \otimes U_{q}(\mathcal{G}) \otimes U_{q}(\mathcal{G}) .
$$

Для построения этого элемента $[26,90,36,91]$ фиксируем некоторый базис $T^{(\alpha)}$ в $U_{q}(\mathcal{G})$. Меж ду $U_{q}(\mathcal{G})$ и $A(\mathbf{G})$ сушествует невырожденное спаривание, которое мы будем обозначать $\langle\ldots\rangle$. Зафиксируем также базис $X^{(\beta)}$ в $A(\mathbf{G})$, ортогональный к $T^{(\alpha)}$ по отношению к этому спариванию. Тогда сумма

$$
\mathbf{T} \equiv \sum_{\alpha} X^{(\alpha)} \otimes T^{(\alpha)} \in A(\mathbf{G}) \otimes U_{q}(\mathcal{G})
$$

как раз и является искомым групповым элементом. Он называется универсальной $\mathbf{T}$ матрицей (поскольку сплетается универсальной $\mathcal{R}$-матрицей) или универсальным групповым элементом.

Для доказательства того, что (4.43) удовлетворяет формуле (4.42), нужно заметить, что матришы $M_{\gamma}^{\alpha \beta}$ и $D_{\beta \gamma}^{\alpha}$, задаюшие законы умножения и коумножения в $U_{q}(\mathcal{G})$, соответственно,

$$
T^{(\alpha)} \cdot T^{(\beta)} \equiv M_{\gamma}^{\alpha \beta} T^{(\gamma)}, \quad \Delta\left(T^{(\alpha)}\right) \equiv D_{\beta \gamma}^{\alpha} T^{(\beta)} \otimes T^{(\gamma)},
$$

индуцируют наоборот соответственно коумножение и умножение в дуальной алгебре $A(\mathbf{G})$ :

$$
\begin{aligned}
D_{\beta \gamma}^{\alpha} & =\left\langle\Delta\left(T^{(\alpha)}\right), X^{(\beta)} \otimes X^{(\gamma)}\right\rangle \equiv\left\langle T^{(\alpha)}, X^{(\beta)} \cdot X^{(\gamma)}\right\rangle, \\
M_{\gamma}^{\alpha \beta} & =\left\langle T^{(\alpha)} T^{(\beta)}, X^{(\gamma)}\right\rangle=\left\langle T^{(\alpha)} \otimes T^{(\beta)}, \Delta\left(X^{(\gamma)}\right)\right\rangle,
\end{aligned}
$$

тогда

$$
\begin{aligned}
\Delta_{U}(\mathbf{T}) & =\sum_{\alpha} X^{(\alpha)} \otimes \Delta_{U}\left(T^{(\alpha)}\right)=\sum_{\alpha, \beta, \gamma} D_{\beta \gamma}^{\alpha} X^{(\alpha)} \otimes T^{(\beta)} \otimes T^{(\gamma)}= \\
& =\sum_{\beta, \gamma} X^{(\beta)} X^{(\gamma)} \otimes T^{(\beta)} \otimes T^{(\gamma)}=\mathbf{T} \otimes_{U} \mathbf{T} .
\end{aligned}
$$

Это свойство является первым определяющим свойством универсального Т-оператора, совпадаюшим с классическим. Второе свойство, которое позволяет рассматривать $\mathbf{T}$ как элемент "настояшей" групाы, - это групповой закон умножения $g \cdot g^{\prime}=g^{\prime \prime}$, заданный отображением

$$
g \cdot g^{\prime} \equiv \mathbf{T} \otimes_{A} \mathbf{T} \in A(\mathbf{G}) \otimes A(\mathbf{G}) \otimes U_{q}(\mathcal{G}) \rightarrow g^{\prime \prime} \in A(\mathbf{G}) \otimes U_{q}(\mathcal{G}) .
$$

Это отображение канонически дается коумножением и снова является универсальным T-оператором:

$$
\begin{aligned}
\mathbf{T} \otimes_{A} \mathbf{T} & =\sum_{\alpha, \beta} X^{(\alpha)} \otimes X^{(\beta)} \otimes T^{(\alpha)} T^{(\beta)}= \\
& =\sum_{\alpha, \beta, \gamma} M_{\alpha, \beta}^{\gamma} X^{(\alpha)} \otimes X^{(\beta)} \otimes T^{(\gamma)}=\sum_{\alpha} \Delta\left(X^{(\alpha)}\right) \otimes T^{(\alpha)},
\end{aligned}
$$


т.е.

$$
\begin{gathered}
g \equiv \mathbf{T}(X, T), \quad g^{\prime} \equiv \mathbf{T}\left(X^{\prime}, T\right), \quad g^{\prime \prime} \equiv \mathbf{T}\left(X^{\prime \prime}, T\right), \\
X \equiv\left\{X^{(\alpha)} \otimes I\right\} \in A(\mathbf{G}) \otimes I, \quad X^{\prime} \equiv\left\{I \otimes X^{(\alpha)}\right\} \in I \otimes A(\mathbf{G}), \\
X^{\prime \prime} \equiv\left\{\Delta\left(X^{(\alpha)}\right)\right\} \in A(\mathbf{G}) \otimes A(\mathbf{G}) .
\end{gathered}
$$

T-оператор для $S L_{q}(2)$. Теперь для получения более компактных формул переопределим генераторы $U_{q}(S L(2))$ так, чтобы получился несимметричный закон коумножения:

$$
\begin{aligned}
T_{+} \rightarrow T_{+} q^{-T_{0}}, & T_{-} \rightarrow q^{T_{0}} T_{-} \\
\Delta\left(T_{+}\right)=I \otimes T_{+}+T_{+} \otimes q^{-2 T_{0}}, & \Delta\left(T_{-}\right)=T_{-} \otimes I+q^{2 T_{0}} \otimes T_{-} .
\end{aligned}
$$

Эта замена приводит к тому, что всюду $q$-число $[n]_{q}$ заменяется на $q$-число

$$
(n)_{q} \equiv \frac{1-q^{2 n}}{1-q^{2}}
$$

а $q$-экспонента $e_{q}(x)$ - на

$$
\mathcal{E}_{q}(x) \equiv \frac{1}{e_{q}(-x)}=\sum_{k \geq 0} \frac{x^{k}}{[k]_{q} !} q^{-k(k-1) / 2}=\sum_{k \geq 0} \frac{x^{k}}{(k)_{q} !} .
$$

Кроме того, разностные операторы

$$
D_{t}^{(\alpha)}=\frac{q^{-\alpha} M_{t}^{+}-q^{\alpha} M_{t}^{-}}{\left(q-q^{-1}\right) t}
$$

заменяются на

$$
D_{t}^{(\alpha)}=\frac{q^{2 \alpha} M_{t}^{+}-1}{\left(q^{2}-1\right) t} .
$$

Фиксируем теперь базис $T^{(\alpha)}=T_{+}^{i} T_{0}^{j} T_{-}^{k}$ в $U_{q}(S L(2))$. Тогда, используя формулу копроизведения для $T^{(\alpha)}$, можно вычислить матрицу $D_{\beta \gamma}^{\alpha}(4.45)$ и явно получить ортонормированный базис $X^{(\alpha)}$ :

$$
X^{(\alpha)}=\frac{x_{+}^{i}}{(i)_{q} !} \frac{x_{0}^{j}}{j !} \frac{x_{-}^{k}}{(k)_{q^{-1}} !},
$$

где порождающие элементы $x_{ \pm}, x_{0}$ образуют борелевскую алгебру Ли

$$
\left[x_{0}, x_{ \pm}\right]=(\log q) x_{ \pm}, \quad\left[x_{+}, x_{-}\right]=0
$$

Таким образом, получаем

$$
\mathbf{T}=\mathcal{E}_{q}^{x_{+} T_{+}} e^{x_{0} T_{0}} \mathcal{E}_{q^{-1}}^{x_{-} T_{-}} .
$$


Общая формула для Т-оператора. В случае общей (простой) алгебры Ли существуют различные явные представления Т-оператора. В [90], например, в соответствии с общей конструкцией (4.43) был рассмотрен так называемый базис Пуанкаре-Биркгоффа-Витта (ПБВ) [89], представленный упорядоченными мономами генераторов алгебры, отвечаюших всем корням. В этом базисе групповой элемент представляется произведением $q$-экспонент типа (4.53), заданным разложением Гаусса, и образующие алгебры функций при этом удовлетворяют соотношениям типа (4.52). В действительности эти соотношения снова задают борелевскую алгебру Ли, что связано с обшей структурой алгебры функций как кодубля (см. [36] и приведенную там ссылку [11]).

Другое интересное представление группового элемента для произвольной простой алгебры Ли было найдено в [91]. Это представление замечательно тем, что оно строится в базисе Шевалле УОА, т.е. на генераторах, отвечающих простым корням. Построить линейньй базис в УОА в терминах генераторов Шевалле для произвольной простой алгебры Ли является трудной задачей, поскольку эти генераторы удовлетворяют дополнительным соотношениям - соотношениям Серра [92], и, следовательно, не являются свободными образуюшими. В работе [91], однако, групповой элемент в этих терминах был построен не путем использования выражений типа (4.43), а прямой проверкой соотношения (4.42) с учетом соотношений Серра.

В [91] групповой элемент был представлен в виде

$$
\begin{aligned}
g & =g_{U} g_{D} g_{L}, \\
g_{U}=\prod_{s}{ }^{<} \mathcal{E}_{q}^{\theta_{s} T_{i(s)}}, \quad g_{L} & =\prod_{s}{ }^{>} \mathcal{E}_{q^{-1}}^{\chi_{s} T_{-i(s)}}, \quad g_{D}=\prod_{i=1}^{r_{\mathrm{G}}} e^{\vec{\phi} \vec{H}},
\end{aligned}
$$

где генераторы $T_{i}$ отвечают только простым корням $\pm \vec{\alpha}_{i}, i=1, \ldots, r_{\mathbf{G}}$, а $<$ и $>$ означают упорядочение сомножителей. Каждый конкретный корень $\vec{\alpha}_{i}$ может появляться в этом произведении несколько раз, так что существуют различные параметризации группового элемента, зависяшие от выбора набора $\{s\}$ и отображения $i(s)$ выбранного набора в множество простых корней. Технические вычисления в терминах генераторов Шевалле оказываются проше, поскольку они имеют особенно простой закон копроизведения:

$$
\begin{aligned}
\Delta\left(T_{i}\right) & =T_{i} \otimes q^{-2 H_{i}}+I \otimes T_{i}, \\
\Delta\left(T_{-i}\right) & =T_{-i} \otimes I+q^{2 H_{i}} \otimes T_{-i} .
\end{aligned}
$$

Заметим, что формула (4.54) выписана для алгебр с корнями одной длины. В общем случае вместо $q$-экспоненты с параметром $q$ необходимо рассматривать $q$-экспоненту с параметром $q^{\left\|\vec{\alpha}_{i}\right\|^{2} / 2}$

Образуюшие алгебры функций $\theta, \chi, \vec{\phi}$ в данной параметризации удовлетворяют квадратичной алгебре, являюшейся экспонентой от алгебры Гейзенберга:

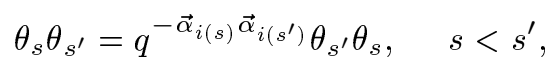

$$
\begin{aligned}
& \chi_{s} \chi_{s^{\prime}}=q^{-\vec{\alpha}_{i(s)} \vec{\alpha}_{i\left(s^{\prime}\right)}} \chi_{s^{\prime}} \chi_{s}, \quad s<s^{\prime}, \\
& e^{\vec{\beta} \vec{\phi}} \theta_{s}=q^{\vec{\beta} \vec{\alpha}_{i(s)}} \theta_{s} e^{\vec{\beta} \vec{\phi}}, \\
& e^{\vec{\beta} \vec{\phi}} \chi_{s}=q^{\vec{\beta} \vec{\alpha}_{i(s)}} \chi_{s} e^{\vec{\beta} \vec{\phi}} .
\end{aligned}
$$

Эти соотношения могут быть найдены из параметризации (4.54) и формулы (4.42). 


\section{4. $\tau$-Функция и представления алгебры функций}

По нашему определению некоммутативная $\tau$-функция - это элемент алгебры функций на группе. Поэтому естественной задачей является фиксировать некоторое представление этой алгебры, а затем определить “значение” соответствующей $\tau$-функции в этом представлении. При этом следует отождествлять каждое представление с некоторым решением интегрируемой иерархии. В действительности именно так в инвариантных терминах должна быть описана группа, действующая в пространстве спектрального параметра [40]. Действительно, конкретное решение классической иерархии 2РТ описывается $\tau$-функцией $(2.1)$ с некоторой конкретной матрицей $\mathcal{G}_{m n}$, задающей элемент грассманниана (2.6). С точки зрения универсального группового элемента это означает, что мы фиксируем некоторое тривиальное представление алгебры функций, которое задается просто $c$-числами. Такие представления исчерпывают представления алгебры функций на классической группе, однако в квантовом случае бывают и нетривиальные представления. Тем не менее естественно по-прежнему отождествлять каждое представление $A(\mathbf{G})$ с некоторым решением иерархии БТ. Естественно, любая редукция интегрируемой системы выделяет некоторое подпространство в пространстве решений, т.е. ограничивает класс рассматриваемых представлений и, как правило, может быть описана некоторой дополнительной групповой структурой (подобно молекуле Тоды см. раздел 3).

Итак, мы пришли к следующей общей алгебраической схеме построения интегрируемой иерархии:

Для любой заданной УОА $U(\mathcal{G})$ при помощи процедуры, описанной в данном разделе, вводится $\tau$-функция, удовлетворяющая БТ и принимающая значения в алгебре функций на группе $A(\mathbf{G})$. При этом задано естественное действие УОА на алгебре функций, а конкретное решение БТ в случае алгебры Ли, отвечаюшее решению соответствуюшей классической иерархии, задается фиксацией представления алгебры функций.

Рассмотрим теперь квантовую $\tau$-функцию, которая является оператором. Тогда возникает естественный вопрос, нельзя ли получить из нее некоторые $c$-числовые величины. Это важно, например, для выявления связи между производяшей функцией корреляторов квантовой системы и $\tau$-функцией. Простейшей $c$-числовой величиной является “двойная" производящая функция, генерирующая матричные элементы как представления, так и копредставления УОА (копредставление УОА эквивалентно по дуальности представлению алгебры функций). Эта производящая функция зависит от четырех наборов времен и должна удовлетворять БТ по отношению к индексам как представления, так и копредставления, т.е. описывать некоторую четырехмерную систему уравнений.

Другой $c$-числовой функцией является сама $\tau$-функция в тривиальном представлении. Этот случай наиболее близок к классическому случаю и потому особенно интересен. Далее мы обсудим этот случай детальнее, однако вначале опишем вкратце структуру копредставлений квантовой группы. Мы рассмотрим простейший пример $S L_{q}(2)$ (для более обшего рассмотрения см. [36] и приведенную там ссылку [11]).

Копредставления УОА $S L_{q}(2)$ даются представлениями алгебры (4.52). Это борелевская алгебра, поэтому она не имеет нетривиальных конечномерных неприводимых представлений [92]. Все конечномерные представления приводимы, но не вполне приво- 
димы. Неприводимые представления были впервые изучены в $[93]^{16)}$. Для сравнения с результатами этой работы перепишем стандартные генераторы $A\left(S L_{q}(2)\right)(4.39)$ в терминах алгебры (4.52) (это своего рода бозонизация алгебры функций):

$$
a=e^{\frac{1}{2} x_{0}}+x_{+} x_{-} e^{-\frac{1}{2} x_{0}}, \quad b=x_{+} e^{-\frac{1}{2} x_{0}}, \quad c=e^{-\frac{1}{2} x_{0}} x_{-}, \quad d=e^{-\frac{1}{2} x_{0}} .
$$

Замечательно, что эти выражения совпадают с (4.30), но различные $x$ при этом не коммутируют.

В этих терминах имеются два типа неприводимых представлений: тривиальное, которое дается $x_{+}=x_{-}=0$, т.е. $a d=1, b=c=0$, и бесконечномерное, которое можно задать явно действием на базис $\left\{e_{k}\right\}_{k \geq 0}[93]$ :

$$
\begin{gathered}
a e_{k}=\left(1-q^{2 k}\right)^{\frac{1}{2}} e_{k-1}\left(a e_{0}=0\right), \quad d e_{k}=\left(1-q^{2 k+2}\right)^{\frac{1}{2}} e_{k+1}, \\
c e_{k}=\theta q^{k} e_{k}, \quad b e_{k}=-\theta^{-1} q^{k+1} e_{k} .
\end{gathered}
$$

Эта структура представлений легко обобщается на другие квантовые группы (ранга $r)$, поскольку алгебры образуюших $x$ всегда борелевские. Таким образом, все множество неприводимых представлений снова исчерпывается тривиальными и бесконечномерными (и те, и другие $r$-параметрические) представлениями. Явные формулы для них могут быть найдены в [94, 95] (см. также [36] и приведенную там ссылку [11]).

$\tau$-Функция в тривиальном представлении и разностная иерархия Каживары-Охты-Сатсумы (KOC). Итак, $\tau$-функция в тривиальном представлении дает некоторый образец $c$-числовой функции. Конечно, эта функция слишком проста и неинтересна. Сейчас, однако, мы получим уравнение, которому удовлетворяет $\tau$-функция в тривиальном представлении и решениями которого являются также уже и вполне нетривиальные $c$-числовые функции.

Заметим вначале, что даже в случае $S L_{q}(2)$ не существует наивного детерминантного представления, аналогичного (2.55). Действительно, введем (для краткости обозначаем $\left.D \equiv D^{(1)}\right)$

$$
C_{1}^{1}=\tau_{F}=a+b \bar{t}+c t+d t \bar{t}
$$

тогда

$$
C_{2}^{1}=D_{\bar{t}} C_{1}^{1}=b+d t, \quad C_{1}^{2}=D_{t} C_{1}^{1}=c+d \bar{t}, \quad C_{2}^{2}=D_{\bar{t}} D_{t} C_{1}^{1}=d
$$

и $C_{b}^{a}$ не может быть отождествлено с образуюшими элементами координатного кольца $S L_{q}(2) a, b, c, d(4.39)$ (например, $C_{2}^{1} C_{1}^{2} \neq C_{1}^{2} C_{2}^{1}$ ). Таким образом, детерминант $\operatorname{det}_{q} C$ не является адекватным объектом и его (или определение $C$ ) необходимо модифицировать, а именно, в случае $S L_{q}(2)$ подходящая формула имеет вид

$$
\tau_{F^{(2)}}=\operatorname{det}_{q} g=1=C_{1}^{1} C_{2}^{2}-q C_{2}^{1} M_{\bar{t}}^{-} C_{1}^{2}=\tau_{F} D_{t} D_{\bar{t}} \tau_{F}-q D_{\bar{t}} \tau_{F} M_{t}^{-} D_{t} \tau_{F} .
$$

$\mathrm{K}$ детерминантным формулам для старших групп мы еше вернемся в следуюшем разделе, а пока заметим, что, если взять $\tau$-функцию в тривиальном представлении

\footnotetext{
${ }^{16)}$ Строго говоря, в [93] изучались *-представления, т.е. представления с дополнительной инволюцией.
} 
$A\left(S L_{q}(2)\right)-a d=1, b=c=0$, она будет удовлетворять более простому уравнению (оно было впервые предложено в $[96,97]$ )

$$
\tau_{F} D_{t} D_{\bar{t}} \tau_{F}-D_{\bar{t}} \tau_{F} D_{t} \tau_{F}=1
$$

Это уравнение имеет вид, близкий к уравнению (3.4), и форму, в отличие от (4.61) легко обобшаемую на $S L_{q}(n)$-случай. Это неудивительно, поскольку уравнение (4.62) решается $\tau$-функцией в тривиальном представлении.

Еще одно соображение в пользу естественности уравнения (4.62) - это то, что оно естественно ведет к детерминанту $q$-полиномов Шура (4.65). Действительно, рассмотрим тривиальный элемент $g$ (отвечающий тривиальному представлению $A(\mathbf{G})$ ). Тогда для случая $S L_{q}(n)$ можно получить прямым вычислением (см. [36] и приведенную там ссылку [5])

$$
\left\langle k, 0, \ldots, 0\left|\mathcal{E}_{q^{-1}}^{s_{2} T_{12}} \mathcal{E}_{q^{-1}}^{s_{3} T_{13}} \ldots \mathcal{E}_{q^{-1}}^{s_{n} T_{1 n}} \mathcal{E}_{q}^{\bar{s}_{2} T_{21}} \mathcal{E}_{q}^{\bar{s}_{3} T_{31}} \ldots \mathcal{E}_{q}^{\bar{s}_{n} T_{n 1}}\right| k, 0, \ldots, 0\right\rangle=P_{k}^{(q)}(s \bar{s})
$$

где $\langle k, 0, \ldots, 0|$ - симметрическое произведение $k$ простейших фундаментальных представлений.

\section{5. Разностная иерархия КОС}

Разностная иерархия КОС из иерархии 2РТ. Теперь рассмотрим уравнение (4.62) более подробно и, в частности, покажем, что оно может быть получено как уравнение классической 2РТ-иерархии с нетривиальной эволюцией [34], т.е. что разностное уравнение $\mathrm{KOC} \mathrm{(4.62)} \mathrm{может} \mathrm{быть} \mathrm{получено} \mathrm{в} \mathrm{рамках} \mathrm{стандартной} \mathrm{дифференииаль-}$ ной $(G L(\infty))$-иерархии 2РТ. Для этого рассмотрим $\tau$-функцию ${ }^{17)}$, определенную просто уравнением (2.24) с переопределенными временными потоками

$$
C_{l}^{k}(s, \bar{s}) \rightarrow \mathcal{C}_{l}^{k}(s, \bar{s})=\sum_{i, j} P_{i-k}^{(q)}(s) R_{i j} P_{j-l}^{(q)}(\bar{s}),
$$

где $q$-полиномы Шура определены формулой

$$
\prod_{i} \mathcal{E}_{q^{i}}\left(s_{i} z^{i}\right)=\sum_{j} P_{j}^{(q)}(s) z^{j}
$$

и удовлетворяют условиям

$$
D_{s_{i}} P_{j}^{(q)}(s)=\left(D_{s_{1}}\right)^{i} P_{j}^{(q)}(s)=P_{j-i}^{(q)}(s) .
$$

Таким образом, мы получаем

$$
D_{s_{i}} \mathcal{C}_{l}^{k}=\mathcal{C}_{l}^{k+i}, \quad D_{\bar{s}_{i}} \mathcal{C}_{l}^{k}=\mathcal{C}_{l+i}^{k}
$$

$$
\tau_{n}^{\left(P^{(q)}\right)}(s, \bar{s} \mid g)=\operatorname{det}_{1 \leq k, l \leq n} D_{s_{1}}^{k-1} D_{\bar{s}_{1}}^{l-1} \mathcal{C}_{1}^{1}(s, \bar{s})
$$

\footnotetext{
17) Как будет показано в следующем разделе, такая $\tau$-функция является производящей функцией матричных элементов в фундаментальных представлениях.
} 
Определенная подобным образом $\tau$-функция, действительно, удовлетворяет уравнениям $(4.62)[96,97,34]$ :

$$
\tau_{k} \cdot D_{s_{1}} D_{\bar{s}_{1}} \tau_{k}-D_{s_{1}} \tau_{k} \cdot D_{\bar{s}_{1}} \tau_{k}=\tau_{k-1} \cdot M_{s_{1}}^{+} M_{\bar{s}_{1}}^{+} \tau_{k+1}
$$

где точки означают остальные уравнения иерархии КОС. Для доказательства этого уравнения проше всего переписать $\tau$-функцию с помошью формулы

$$
\operatorname{det} D_{s_{1}}^{i} D_{\bar{s}_{1}}^{j} C=q^{-(n-1)(n-2)}(1-q)^{n(n-1)}(t \bar{t})^{\frac{n(n-1)}{2}} \operatorname{det}_{0 \leq i, j<n}\left(M_{s_{1}}^{+}\right)^{i}\left(M_{\bar{s}_{1}}^{+}\right)^{j} C
$$

а затем применить к ней тождество Якоби ${ }^{18)}$.

Фермионный язык для иерархии КОС. В заключение данного раздела покажем, как иерархия КОС может быть переписана на фермионном языке. Поскольку ее $\tau$-функция получается из $\tau$-функции иерархии Тоды переопределением времен, достаточно просто подставить в $\tau$-функцию иерархии $2 \mathrm{PT}$ новые времена. Действительно, формула

$$
\prod_{k=1}^{\infty} \mathcal{E}_{q^{k}}\left(s_{k} z^{k}\right)=\prod_{k=1}^{\infty} e^{t_{k} z^{k}}
$$

позволяет выразить времена $t$ через $s$ :

$$
\sum_{k=1}^{\infty} t_{k} z^{k}=\sum_{n, k=1}^{\infty} \frac{s_{k}^{n}\left(1-q_{k}\right)^{n}}{n\left(1-q_{k}^{n}\right)} z^{n k}
$$

причем

$$
P_{k}^{(q)}(s)=P_{k}(t)
$$

Таким образом, $\tau$-функцию можно представить в виде

$$
\tau_{n}(s, \bar{s} \mid g)=\tau_{n}(t, \bar{t} \mid g) \stackrel{(2.1)}{\sim}\left\langle n\left|e^{H(t)} g e^{\bar{H}(\bar{t})}\right| n\right\rangle,
$$

18) Тождество Якоби является частным $(p=2)$ случаем общего тождества на миноры произвольной матрицы

$$
\sum_{i_{p}} C_{r i_{p}} \widehat{C}_{i_{1} \ldots i_{p} \mid j_{1} \ldots j_{p}}=\frac{1}{p !} \sum_{P}(-)^{P} \widehat{C}_{i_{1} \ldots i_{p-1} \mid j_{P(1)} \ldots j_{P(p-1)}} \delta_{r j_{P(p)}},
$$

где сумма в правой части идет по всем перестановкам $p$ индексов, а $\widehat{C}_{i_{1} \ldots i_{p} \mid j_{1} \ldots j_{p}}$ обозначает детерминант (минор) матрицы, которая получается из $C_{i j}$ выбрасыванием строк $i_{1} \ldots i_{p}$ и столбцов $j_{1} \ldots j_{p}$. Используя тот факт, что $\left(C^{-1}\right)_{i j}=\widehat{C}_{i \mid j} / \widehat{C}$, можно переписать это тождество в виде

$$
\widehat{C} \widehat{C}_{i_{1} \ldots i_{p} \mid j_{1} \ldots j_{p}}=\left(\frac{1}{p !}\right)^{2} \sum_{P, P^{\prime}}(-)^{P}(-)^{P^{\prime}} \widehat{C}_{i_{P^{\prime}(1)} \ldots i_{P^{\prime}(p-1)} \mid j_{P(1)} \ldots j_{P(p-1)}} \delta_{i_{P^{\prime}(p)} \mid j_{P(p)}} .
$$

2 Теоретическая и математическая физика, т. 114, № 2, 1998 г. 
где

$$
\begin{aligned}
& H(t)=\sum_{n>0} t_{n} J_{+n} \stackrel{(4.72)}{=} \sum_{n, k=0}^{\infty} \frac{s_{k}^{n}\left(1-q_{k}\right)^{n}}{n\left(1-q_{k}^{n}\right)} J_{+n k}, \\
& \bar{H}(\bar{t})=\sum_{n>0} \bar{t}_{n} J_{-n}=\sum_{n, k=0}^{\infty} \frac{\bar{s}_{k}^{n}\left(1-q_{k}\right)^{n}}{n\left(1-q_{k}^{n}\right)} J_{-n k} .
\end{aligned}
$$

$\tau$-Функция иерархии КОС может также быть рассмотрена как $\tau$-функция $2 \mathrm{PT}$-иерархии в переменных Мивы [34]. Для этого нужно рассмотреть общее преобразование Мивы в специальных точках, а именно, с помошью формул типа

$$
t_{k}=\frac{1}{k} \frac{\left((1-q) s_{1}\right)^{k}}{1-q^{k}}=\frac{1}{k} \sum_{l \geq 0}\left((1-q) q^{l} s_{1}\right)^{k}
$$

легко получить, что $\tau$-функция (4.74) описывается следуюшим набором переменных Мивы:

$$
\left\{e^{2 \pi i a / k} \mu_{k} q_{k}^{-l / k} \mid a=0, \ldots, k-1 ; l \geq 0\right\}, \quad \mu_{k}=\left(\left(1-q_{k}\right) s_{k}\right)^{-1 / k} .
$$

Это означает, что иерархию КОС можно рассматривать как иерархию 2РТ в переменных Мивы при специальном выборе (4.77) этих последних.

\section{5. КВАНТОВАЯ И КЛАССИЧЕСКАЯ ИЕРАРХИИ КП С РАЗЛИЧНЫМИ ЭВОЛЮЦИЯМИ}

\section{1. Структура фундаментальных представлений}

В данном разделе мы исследуем частный случай рассмотренной выше конструкции интегрируемые иерархии, отвечаюшие фундаментальным представлениям групп $S L(p)$ и $S L_{q}(p)[35-38]$. Эти случаи наиболее важны, поскольку именно они отвечают стандартной иерархии 2РТ и ее квантовому аналогу, и именно в этих случаях сушествуют детерминантные представления для $\tau$-функций. Заметим, что в квантовом случае для получения детерминантных представлений мы несколько расширяем рамки определения $\tau$-функции (4.2), вводя некоммутативные времена.

Если говорить строго, иерархия 2 РТ описывается группой $S L(\infty)$. Однако мы будем рассматривать случай общего $p$, хотя результаты зависят от $p$ слабо.

Начнем с описания самих фундаментальных представлений этих групп [92] (см. также приложение 2 ). Группа $S L(p)$ имеет $r \equiv$ rank $=p-1$ фундаментальных представлений. Простейшее из них $F \equiv F_{1}$ есть $p$-плет, содержащий состояния

$$
\psi_{i}=T_{-}^{i-1}|0\rangle, \quad i=1, \ldots, p .
$$

Здесь генератор $T_{-}$является суммой всех $r$ nросты $x$ корней $S L(p): T_{-}=\sum_{i=1}^{r} T_{-\alpha_{i}}$. Все остальные фундаментальные представления $F_{k}$ теперь могут быть построены как антисимметричная степень $F=F_{1}$ :

$$
F^{(k)}=\left\{\Psi_{i_{1} \ldots i_{k}}^{(k)} \sim \psi_{\left[i_{1}\right.} \ldots \psi_{\left.i_{k}\right]}\right\} .
$$


$F_{k}$ задается действием операторов

$$
R_{k}\left(T_{-}^{i}\right) \equiv T_{-}^{i} \otimes I \otimes \cdots \otimes I+I \otimes T_{-}^{i} \otimes \cdots \otimes I+I \otimes I \otimes \cdots \otimes T_{-}^{i}
$$

на вектор старшего веса. Эти операторы коммутируют друг с другом. Очевидно, что для данного $k$ в точности $k$ из них (с $i=1, \ldots, k)$ независимы. Тот факт, что все фундаментальные представления генерируются тем же самым генератором $T_{-},-$замечательное свойство, которое может послужить определением фундаментальных представлений и ответственно за все сушественные свойства классических интегрируемых иерархий, в частности за наличие большого числа коммутирующих гамильтонианов.

Теперь можно явно построить либо пару сплетаюших операторов, аналогичных операторам пункта 4.2 :

$$
I_{(k)}: F_{k+1} \rightarrow F_{k} \otimes F, \quad I_{(k)}^{*}: F_{k-1} \rightarrow F^{*} \otimes F_{k},
$$

так что

$$
\Gamma_{k \mid k^{\prime}}: F_{k+1} \otimes F_{k^{\prime}-1} \rightarrow F_{k} \otimes F_{k^{\prime}},
$$

либо пару фермионных сплетаюших операторов

$$
\psi^{+}: F_{1} \otimes F_{k} \rightarrow F_{k+1}, \quad \psi^{-} \equiv I_{(k)},
$$

здесь

$$
\begin{gathered}
F^{*}=F^{(r)}=\left\{\psi^{i} \sim \epsilon^{i i_{1} \ldots i_{r}} \psi_{\left[i_{1} \ldots \psi_{\left.i_{r}\right]}\right.}\right\}, \\
I_{(k)}: \Psi_{i_{1} \ldots i_{k+1}}^{(k+1)}=\Psi_{\left[i_{1} \ldots i_{k}\right.}^{(k)} \psi_{\left.i_{k+1}\right]}, \quad I_{(k)}^{*}: \Psi_{i_{1} \ldots i_{k-1}}^{(k-1)}=\Psi_{i_{1} \ldots i_{k-1} i}^{(k)} \psi^{i},
\end{gathered}
$$

а $\Gamma_{k \mid k^{\prime}}$ сконструирован с помощью вложения $I \rightarrow F \otimes F^{*}$, индуцированного спариванием $\psi_{i} \psi^{i}$. Базис в линейном пространстве $F^{(k+1)} \otimes F^{\left(k^{\prime}-1\right)}$ индуцирован оператором $\Gamma_{k \mid k^{\prime}}$ из базиса в пространстве $F^{(k)} \otimes F^{\left(k^{\prime}\right)},-$ это $\Psi_{\left[i_{1} \ldots i_{k}\right.}^{(k)} \Psi_{\left.i_{k+1}\right] i_{1}^{\prime} \ldots i_{k^{\prime}-1}^{\prime}}^{\left(k^{\prime}\right)}$.

Теперь можно переписать оператор Г в терминах матричных элементов

$$
g^{(k)}\left(\begin{array}{c}
i_{1} \ldots i_{k} \\
j_{1} \ldots j_{k}
\end{array}\right) \equiv\left\langle\Psi_{i_{1} \ldots i_{k}}|g| \Psi_{j_{1} \ldots j_{k}}\right\rangle=\operatorname{det}_{1 \leq a, b \leq k} g_{j_{b}}^{i_{a}}
$$

следуюшим образом:

$$
\begin{aligned}
& g^{(k)}\left(\begin{array}{c}
i_{1} \ldots i_{k} \\
{\left[j_{1} \ldots j_{k}\right.}
\end{array}\right) g^{\left(k^{\prime}\right)}\left(\begin{array}{c}
i_{1}^{\prime} \ldots i_{k}^{\prime} \\
\left.j_{k+1}\right] j_{1}^{\prime} \ldots j_{k^{\prime}-1}^{\prime}
\end{array}\right)= \\
& =g^{(k+1)}\left(\begin{array}{c}
i_{1} \ldots i_{k}\left[i_{k^{\prime}}^{\prime}\right. \\
j_{1} \ldots j_{k+1}
\end{array}\right) g^{\left(k^{\prime}-1\right)}\left(\begin{array}{c}
\left.i_{1}^{\prime} \ldots i_{k^{\prime}-1}^{\prime}\right] \\
j_{1}^{\prime} \ldots j_{k^{\prime}-1}^{\prime}
\end{array}\right) .
\end{aligned}
$$

Это явная форма БТ (4.10) для матричных элементов в случае фундаментальных представлений, верная тождественно для любых $g^{(k)}$ вида (5.7). "Одевая" матричные элементы $g^{(k)}$ полиномами Шура, зависящими от времен, нетрудно получить из (5.7) уравнение (2.24), а из (5.8) - уравнение двумеризованной модели Тоды (3.4). Ниже, однако, мы будем всюду использовать БТ в УОА и фермионную пару сплетающих операторов (5.5). 
Заметим, что фундаментальные представления квантовой группы $S L_{q}(p)$ имеют такую же структуру, только антисимметризация должна быть всюду заменена $q$-антисимметризацией. Некоторые подробности можно найти в приложении 2 и [35].

Перейдем теперь к более инвариантной записи. А именно, заметим, что, как описано выше, фундаментальные представления полностью генерируются операторами

$$
T_{ \pm}^{(k)}=\sum_{\vec{\alpha}: h(\vec{\alpha})=k} T_{ \pm \vec{\alpha}}
$$

- суммами всех генераторов $S L(p)$, ассоциированными с положительными/отрицательными корнями "веса" $k$ (в первом фундаментальном представлении $F$ такие $T_{ \pm}^{(k)}$ являются матрицами размера $p \times p$ с единицами на $k$-й верхней/нижней диагонали и нулями в остальных местах).

Мы уже отмечали в пункте 4.1, что эволюционные операторы $U(t), \bar{U}(\bar{t})$ в представлениях обшего вида должны содержать все повышаюшие и соответственно понижающие операторы ${ }^{19)}$, а также отвечать некоммутируюшим потокам. В случае же фундаментальных представлений $F_{n}$ они могут быть выбраны в виде

$$
\begin{aligned}
U(t) & =\exp \left(\sum_{k \geq 1} t_{k} T_{+}^{(k)}\right), \\
\bar{U}(\bar{t}) & =\exp \left(\sum_{k \geq 1} \bar{t}_{k} T_{-}^{(k)}\right) .
\end{aligned}
$$

Сушественным свойством операторов $T_{ \pm}^{(k)}$ является их коммутативность:

$$
\left[T_{+}^{(k)}, T_{+}^{(l)}\right]=0, \quad\left[T_{-}^{(k)}, T_{-}^{(l)}\right]=0,
$$

поэтому $U(t), \bar{U}(\bar{t})(5.9)$ отвечают коммутирующим потокам. Конечно, эволюционные операторы обшего вида зависят от большего числа времен и взаимно некоммутируюших генераторов группы.

Операторы, определенные в (5.9), имеют следующие свойства:

1) $U, \bar{U} \in S L(p)$;

2 ) конкретнее, $U, \bar{U}$ принадлежат нильпотентной подгруппе $N S L(p)$ группы $S L(p)$. На самом деле, $N S L(p)$ является подгруппой борелевской подгруппы: $N S L(p) \subset$ $B S L(p) \subset S L(p)$ (в фундаментальном представлении $F_{1}$ подгруппа $B S L(p)$ состоит из всех верхнетреугольных матриц с единичным определителем, в то время как матрицы из $N S L(p)$ имеют, кроме того, единичные элементы на главной диагонали);

3) поскольку копроизведение задается формулой

$$
\Delta\left(T_{ \pm \vec{\alpha}}\right)=T_{ \pm \vec{\alpha}} \otimes I+I \otimes T_{ \pm \vec{\alpha}}
$$

TO

$$
\Delta U(t)=U(t) \otimes U(t)=(U(t) \otimes I)(I \otimes U(t)) .
$$

${ }^{19)}$ Поскольку $\tau$-функция по определению является производящей функцией всех матричных элементов. 
Иными словами, эволюционные операторы $U, \bar{U}$ являются групповыми элементами подгруппы $N S L(p)$.

Описанные свойства являются весьма существенными, и естественно попытаться сохранить их при квантовании. При этом необходимо учесть два обстоятельства.

Во-первых, не существует ничего подобного операторам $T_{ \pm}^{(k)}$ при $q \neq 1$ (по крайней мере, сформулированным в виде, не зависяшем от представления $R$ ). Это означает, что явные выражения для $U(t)$ и $\bar{U}(\bar{t})$ должны сильно отличаться от (5.9).

Во-вторых, не существует естественного определения нильпотентной подгруппы $N \mathbf{G}_{q}$ в квантовом случае: хорошо определена только квантовая деформация борелевской подгруппы $B \mathbf{G}_{q} \subset \mathbf{G}_{q}$ (см. пункт 4.3). Действительно, если выбрать в качестве $U(t)$ объект вида $g_{U}$, а $\bar{U}(\bar{t})$ - вида $g_{L}$ (см. (4.54)), то из-за отсутствия фактора $g_{D}$ имеем $\Delta\left(g_{U}\right) \neq g_{U} \otimes g_{U}, \Delta\left(g_{L}\right) \neq g_{L} \otimes g_{L}$ и нильпотентной подгруппы $N \mathbf{G}_{q}$, действительно, не сушествует. В то же время борелевская подгруппа $B \mathbf{G}_{q}$ сушествует, поскольку $\Delta\left(g_{U} g_{D}\right)=\left(g_{U} g_{D}\right) \otimes\left(g_{U} g_{D}\right)$.

Несмотря на эту проблему мы будем рассматривать $U$ и $\bar{U}$ как величины типа $g_{U}$ и $g_{L}$, соответственно (см., однако, конец данного раздела). По этой причине уравнение (5.12) в квантовом случае требует некоторой модификации. Точнее, вместо (5.12) мы получим

$$
\Delta(U(\xi))=U_{L}^{(2)}(\xi) \cdot U_{R}^{(2)}(\xi)
$$

где

$$
\begin{aligned}
& U(\xi)=\prod_{s}^{<} \mathcal{E}_{q}\left(\xi_{s} T_{i(s)}\right),
\end{aligned}
$$

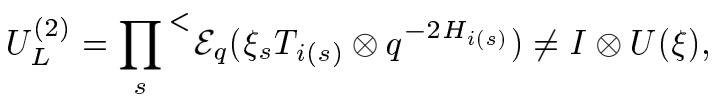

$$
\begin{aligned}
& U_{R}^{(2)}=\prod_{s}^{<} \mathcal{E}_{q}\left(\xi_{s} I \otimes T_{i(s)}\right)=I \otimes U(\xi) .
\end{aligned}
$$

Это выражение далее будет использовано для вывода детерминантных формул для квантовой $\tau$-функции.

Заметим, что, рассматривая эволюционные операторы как элементы разложения (4.54), мы принимаем новый подход к понятию обобщенной $\tau$-функции, когда не только групповой элемент, но и операторы $U$ и $\bar{U}$ являются элементами $U(\mathcal{G}) \otimes A(\mathbf{G})$, т.е. времена являются некоммутативными параметрами. Именно этот подход позволяет построить детерминантные представления для $\tau$-функций.

Подчеркнем, что в рамках этой логики здесь и далее под умножением эволюционных операторов $U$ и $\bar{U}$ и группового элемента $g$ в определении $\tau$-функции (4.2) мы всегда понимаем групповой закон умножения (4.47), т.е. элементы алгебры $\theta, \phi$ и $\chi(4.56)$ в эволюционных операторах коммутируют с элементами соответствующей алгебры в $g$ (см. пункт 4.3).

\section{2. Параметризации группового элемента в фундаментальных представлениях}

Вернемся теперь к общей параметризации (4.54) группового элемента, заданного в терминах генераторов Шевалле, и рассмотрим случай группы $S L(p)$. Тогда наиболее 
простой способ выбрать отображение $i(s)$ это

$$
i(s): 1,2, \ldots, r-1, r ; 1,2, \ldots, r-1 ; 1,2,3 ; 1,2 ; 1
$$

с $s=1, \ldots, p(p-1) / 2($ размерность группы $S L(p))$, т.е.

$$
U(\xi)=\prod_{1 \leq i \leq p} \prod_{i<j \leq p} \exp \left(\xi_{i j} T_{j-i}\right) .
$$

Поскольку, однако, мы собираемся изучать фундаментальные представления, то, как мы видели в предыдущем пункте, достаточно рассмотреть орбиты $S L(p)$, параметризованные только $r$ переменными. Поэтому далее нашей целью будет найти адекватную параметризацию таких орбит. В классическом случае $(q=1)$ существуют по меньшей мере три такие параметризации, рассматриваемые ниже [37, 38]. Однако только одна из них допускает простую квантовую деформацию, причем эта параметризация не отвечает выражению (5.9). Сложность построения таких орбит меньшей размерности в квантовом случае связана с необходимостью согласования редукции с коммутационными соотношениями (4.56), т.е. необходимостью выбора подкласса весьма специальных представлений алгебры функций на квантовой группе.

Параметризация А. Простейшей возможностью параметризации А являются просто ограничение набора $\{s\}$ до $s=1, \ldots, r$ и выбор $i(s)=s$, т.е.

$$
U^{(A)}(\xi)=\prod_{i=1}^{r_{\mathbf{G}}<} \exp \left(\xi_{i} T_{i}\right)
$$

Этого достаточно, чтобы генерировать все состояния любого фундаментального представления из соответствуюшего вакуумного вектора (вектора старшего веса), но $\left\langle 0_{F_{n}}\right| U^{(A)}(\xi)$ имеет мало обшего с элементом $\left\langle 0_{F_{n}}\right| U(t)$ (с $U(t)$, заданным формулой (5.9)). Возможно, лучше говорить, что идентификация $\left\langle 0_{F_{n}}\right| U^{(A)}(\xi)=\left\langle 0_{F_{n}}\right| U(t)$ задает весьма сложное отображение $\xi_{i}(t)$, которое явно зависит от $p$.

Естественно, можно построить иерархию КП/Тоды в терминах переменных $\xi$ вместо обычных времен $t$ (см. ниже), но она не получается просто как замена переменных, вся конструкция выглядит совершенно иной. Это цена того, что новая конструкция непосредственно обобщается на случай $q \neq 1$ : вместо (5.17) нужно просто написать

$$
U^{(A)}(\xi)=\prod_{i=1}^{r_{\mathbf{G}}<} \mathcal{E}_{q}\left(\xi_{i} T_{i}\right)
$$

где $\xi$ - некоммутирующие переменные,

$$
\xi_{i} \xi_{j}=q^{-\vec{\alpha}_{i} \vec{\alpha}_{j}} \xi_{j} \xi_{i}, \quad i<j .
$$

Теперь, как будет показано ниже, любое утверждение при $(q=1)$ в $\xi$-параметризации легко обобщается на случай $q \neq 1$. 
Параметризация В (стандартная). Другой выбор параметризации связан со стандартными временами иерархии КП/Тоды:

$$
U^{(B)}(\xi)=\prod_{s} \exp \left(\xi_{s} T_{i(s)}\right)=U(t)=\exp \left(\sum_{k} t_{k} T_{+}^{(k)}\right) .
$$

Это подразумевает, что $\left\langle 0_{F_{n}}\right| U^{(B)}(\xi)=\left\langle 0_{F_{n}}\right| U(t)$ с некоторыми не зависящими от $p$ функциями $\xi_{s}(t)$. Ключевая разница между двумя частями равенства (5.20) заключается в следующем. В то время как правая часть содержит взаимнокоммутирующие комбинации генераторов, отвечающих непростым корням, левая часть содержит только взаимнонекоммутирующие генераторы Шевалле. Такая репараметризация, действительно, существует, однако набор $\{s\}$ должен при этом содержать по меньшей мере $p(p-1) / 2$ элементов, и можно выбрать $i(s)$ просто, как в (5.16). Однако теперь не все $\xi_{s}$ являются независимыми, вместо этого они выражаются через $r$ времен $t_{k}$. K примеру, $t_{1}$-зависимость $\xi_{i j}$ дается формулой

$$
\xi_{i j}=\frac{t_{1}}{p+i-j}+\mathcal{O}\left(t_{2}, t_{3}, \ldots\right) .
$$

Проблемой, однако, является построение разумной квантовой деформации параметризации В. Действительно, при этом необходимо воспроизвести правильные коммутационные соотношения

$$
\xi_{s} \xi_{s^{\prime}}=q^{-\vec{\alpha}_{i(s)} \vec{\alpha}_{i\left(s^{\prime}\right)}} \xi_{s^{\prime}} \xi_{s}, \quad s<s^{\prime},
$$

для $p(p-1) / 2$ переменных $\xi_{s}$ как следствие некоторых соотношений между $r$ переменными $t_{k}$ (которые, конечно, не коммутируют при $q \neq 1$ ). Для этого также необходимо некоторым образом деформировать соотношения (5.21) при $q \neq 1$. Решение этой проблемы до сих пор не найдено.

Параметризация C (переменные Мивы). Имеется еше одна возможность сделать (не зависяшее от представления) преобразование Мивы стандартных времен

$$
t_{k}=\frac{1}{k} \sum_{a} \lambda_{a}^{k}
$$

Это преобразование оказывается замечательно согласовано с разложением по простым корням:

$$
U(t)=\prod_{a} \exp \left(\sum_{k=1}^{r_{\mathbf{G}}} \frac{\lambda_{a}^{k}}{k} T_{+}^{(k)}\right)=\prod_{a}\left(\prod_{i=1}^{r_{\mathbf{G}}} e^{\lambda_{a} T_{i}}\right) .
$$

Набор $\{s\}$ и отображение $i(s)$ не являются наиболее простой реализацией формулы (5.16), но общее правило (4.56) при квантовой деформации, конечно, применимо.

В данной параметризации проблема состоит в том, что (5.16) подразумевает квантовую формулу вида

$$
\prod_{a}\left(\prod_{i=1}^{r_{\mathbf{G}}} \mathcal{E}_{q}\left(\lambda_{a i} T_{i}\right)\right)
$$

где $\lambda_{a i}$ с разными $i$ и тем же самым $a$ не коммутируют. В то же время условие $\lambda_{a i}=\lambda_{a j}$ при $i \neq j$ крайне важно для классической $(q=1)$ формулы (5.23). Что же является правильной квантовой деформацией этого условия, остается неясным. 


\section{3. Классическая $(q=1)$ иерархия КП/Тоды в разных параметризациях}

Детерминантные формулы и системы уравнений. Для лучшего понимания развиваемой в данном обзоре схемы вновь выведем теперь стандартные свойства классических интегрируемых иерархий в рамках рассматриваемой здесь групповой интерпретации. К этим стандартным свойствам относятся детерминантные представления для $\tau$-функций и набор дифференциальных БТ. Мы рассмотрим случай фундаментальных представлений группы $S L(p)$, поскольку именно в этом случае имеются детерминантные представления для $\tau$-функций. Для их вывода нет необходимости фиксировать как-либо параметризацию, и можно рассмотреть произвольную эволюцию $U(t)$.

Итак, мы будем рассматривать $\tau$-функции $\tau_{n} \equiv \tau_{F_{n}}(t, \bar{t} \mid g)$. Начнем с простейшей из них

$$
\tau_{1}=\left\langle 0_{F_{1}}|U(t) g \bar{U}(\bar{t})| 0_{F_{1}}\right\rangle .
$$

Заметим, что специфической чертой $F=F_{1}$ является то, что

$$
\left\langle 0_{F}\right| U(t)=\sum_{k} \mathcal{P}_{k}(t)\left\langle 0_{F}\right| T_{+}^{k}=\sum_{k} \mathcal{P}_{k}(t)\left\langle k_{F}\right|,
$$

где правая часть равенства представляет собой переразложение в терминах "обобщенных полиномов Шура" (первое равенство в этой формуле служит их определением) и $p$ состояний $F=F_{1}$ обозначены как $\left\langle k_{F}\right|=\left\langle 0_{F}\right| T_{+}^{k}, k=0, \ldots, r=p-1$. Таким образом, имеем

$$
\begin{aligned}
\tau_{1}(t, \bar{t} \mid g) & =\sum_{k, \bar{k}} \mathcal{P}_{k}(t) \mathcal{P}_{\bar{k}}(\bar{t})\left\langle 0_{F}\left|T_{+}^{k} g T_{-}^{\bar{k}}\right| 0_{F}\right\rangle= \\
& =\sum_{k, \bar{k}} \mathcal{P}_{k}(t) \mathcal{P}_{\bar{k}}(\bar{t})\left\langle k_{F}|g| \bar{k}_{F}\right\rangle=\sum_{k, \bar{k}} \mathcal{P}_{k}(t) g_{k, \bar{k}} \mathcal{P}_{\bar{k}}(\bar{t}) .
\end{aligned}
$$

Определим также

$$
\tau_{1}^{m \bar{m}} \equiv\left\langle m_{F}|U(t) g \bar{U}(\bar{t})| \bar{m}_{F}\right\rangle=\sum_{k, \bar{k}} \mathcal{P}_{k}(t) g_{m+k, \bar{m}+\bar{k}} \mathcal{P}_{\bar{k}}(\bar{t}) .
$$

Вернемся теперь к общему фундаментальному представлению $F_{n}$. Поскольку

$$
\begin{aligned}
\left\langle m_{1} \ldots m_{n F_{n}}\right|= & \left\langle m_{1 F}\right| \otimes\left\langle m_{2 F}\right| \otimes \cdots \otimes\left\langle m_{n F}\right|+ \\
& + \text { антисимметризация по } m_{1}, \ldots, m_{n}= \\
= & \sum_{P}(-)^{P}\left\langle m_{P(1)}\right| \otimes\left\langle m_{P(2)}\right| \otimes \cdots \otimes\left\langle m_{P(n-1)}\right|,
\end{aligned}
$$

то вакуум (состояние старшего веса) $F_{n}$ можно записать в виде

$$
\left\langle 0_{F_{n}}\right|=\left\langle 0,1, \ldots, n-1_{F_{n}}\right|=\sum_{P}(-)^{P}\left\langle P(0)_{F}\right| \otimes\left\langle P(1)_{F}\right| \otimes \cdots \otimes\left\langle P(n-1)_{F}\right| .
$$

Так как для классической группы (см. (5.12)) имеем

$$
\left.U(t)\right|_{F_{n}}=\Delta^{n-1} U(t)=U(t)^{\otimes n},\left.\quad g\right|_{F_{n}}=\Delta^{n-1}(g)=g^{\otimes n},
$$


то окончательно получаем

$$
\begin{aligned}
\tau_{n+1}(t, \bar{t} \mid g) \equiv & \left\langle 0_{F_{n}}|U(t) g \bar{U}(\bar{t})| 0_{F_{n}}\right\rangle= \\
& =\sum_{P, \bar{P}}(-)^{P}(-)^{\bar{P}} \prod_{k=0}^{n}\left\langle P(k)_{F}|U(t) g \bar{U}(\bar{t})| \bar{P}(k)_{F}\right\rangle= \\
& =\operatorname{det}_{0 \leq m, \bar{m}<n} \tau_{1}^{m \bar{m}}=\operatorname{det}_{0 \leq m, \bar{m}<n} \sum_{l, \bar{l}} \mathcal{P}_{l-m}(t) g_{l_{F}, \bar{l}_{F}} \mathcal{P}_{\bar{l}-\bar{m}}(\bar{t})= \\
& =\sum_{\substack{1<m_{1}<m_{2}<\cdots \\
1<\bar{m}_{1}<\bar{m}_{2}<\cdots}} \operatorname{det}_{j i} \mathcal{P}_{m_{j}-i}(t) \operatorname{det}_{j i} g_{m_{j} \bar{m}_{i}} \operatorname{det}_{i j} \mathcal{P}_{\bar{m}_{i}-\bar{j}}(\bar{t}) .
\end{aligned}
$$

Этот результат следует сравнивать с формулой (2.24) при $p \rightarrow \infty$, причем соответствующая диаграмма Дынкина группы $A_{p-1}$ при взятии этого предела должна быть сделана бесконечной в обе стороны (полубесконечной для полубесконечной иерархии).

Следуюшим шагом является получение дифференциальных БТ. Конечно, детерминантные формулы (5.30) являются не лучшей стартовой точкой для этого. Гораздо удобнее использовать методы, основанные на применении сплетающих операторов, которые мы развивали в данном обзоре. В частности, для фундаментальных представлений следует рассмотреть фермионную пару $\psi_{i}^{ \pm}(i=1, \ldots, p)$ сплетающих операторов $\psi^{ \pm}: F_{1} \otimes F_{n} \rightleftarrows F_{n+1}$. Тогда вывод БТ для произвольного $p$ почти буквально совпадает с выводом БТ для $p \rightarrow \infty$, описанным в пункте 4.1. Следует только ввести фермионы, содержащие конечное число степеней спектрального параметра $\psi^{+}(z) \equiv \sum_{i=1}^{p} \psi_{i}^{+} z^{i}$ и $\psi^{-}(z) \equiv \sum_{i=1}^{p} \psi_{i}^{-} z^{p-i+1}$, и соответственно определить вершинные операторы формулами

$$
\sum_{i} \Psi_{k}^{+, i}(t) z^{i} \equiv \widehat{X}^{+}(z, t) \tau_{n}(t), \quad \sum_{i} \Psi_{k}^{-, i}(t) z^{p-i+1} \equiv \widehat{X}^{-}(z, t) \tau_{n}(t)
$$

и аналогично для $\widehat{\bar{X}}^{ \pm}(z, t)$. При этом БТ принимают вид $[37,38]$

$$
\begin{aligned}
& \oint \frac{d z}{z^{p+2}} \widehat{X}^{-}(z, t) \tau_{n}(t, \bar{t}) \widehat{X}^{+}\left(z, t^{\prime}\right) \tau_{m}\left(t^{\prime}, \bar{t}^{\prime}\right)= \\
& \quad=\oint \frac{d z}{z^{p+2}} \widehat{\bar{X}}^{-}(z, \bar{t}) \tau_{n+1}(t, \bar{t}) \widehat{\bar{X}}^{+}\left(z, \bar{t}^{\prime}\right) \tau_{m-1}\left(t^{\prime}, \bar{t}^{\prime}\right) .
\end{aligned}
$$

Для получения конкретных систем дифференциальных уравнений теперь нужно рассмотреть частные выборы параметризаций $U(t), \bar{U}(\bar{t})$.

Параметризация В (стандартная). Выбор этой параметризации приводит к стандартной иерархии КП/Тоды с детерминантным представлением $(2.24),(2.25)$ для $\tau$-функции. Используем выражение (5.9). Это дает

$$
\left\langle 0_{F}\right| U(t)=\left\langle 0_{F}\right| \exp \left(\sum_{k} t_{k} T_{+}^{(k)}\right)=\sum_{k} P_{k}(t)\left\langle k_{F}\right|
$$


со стандартными полиномами Шура $P_{k}(t)(2.15)$. Основной особенностью рассматриваемой эволюции является свойство

$$
\tau_{1}^{m \bar{m}}=\frac{\partial}{\partial t_{m}} \frac{\partial}{\partial \bar{t}_{\bar{m}}} \tau_{1}=\left(\frac{\partial}{\partial t_{1}}\right)^{m}\left(\frac{\partial}{\partial \bar{t}_{1}}\right)^{\bar{m}} \tau_{1} .
$$

Для получения системы уравнений в данной параметризации достаточно заметить, что вершинные операторы (2.33), (2.34) в ней принимают вид

$$
\begin{aligned}
& \widehat{X}^{+}(z, t)=\operatorname{Pr}_{p}\left[e^{\xi(z, t)} \operatorname{Pr}\left[z^{n} e^{-\xi\left(z^{-1}, \tilde{\partial}_{t}\right)}\right]\right], \\
& \widehat{X}^{-}(z, t)=\operatorname{Pr}_{p}\left[e^{\xi(z, t)} \operatorname{Pr}\left[z^{p-n+1} e^{\xi\left(z^{-1}, \tilde{\partial}_{t}\right)}\right]\right]
\end{aligned}
$$

(и аналогично для другой пары вершинных операторов), где $\operatorname{Pr}[f(z)]$ обозначает про-

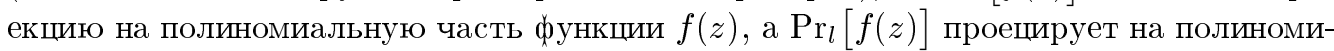
альную часть степени $l$.

Подставляя эти формулы в (5.32) и разлагая эту последнюю по степеням $t_{i}-t_{i}^{\prime}$, мы приходим к набору уравнений, который при $p \rightarrow \infty$ дает уравнения обычной иерархии 2РТ [62].

Иерархия КП/Тоды в параметризации А. Рассмотрим теперь ту же иерархию с другой эволюцией, заданной параметризацией А. Обозначим далее для краткости $\widehat{U}(\xi) \equiv U^{(A)}(\xi)$, а соответствуюшую $\tau$-функцию - $\hat{\tau}(\xi, \bar{\xi} \mid g)$. Эта $\tau$-функция линейна по любому времени $\xi_{i}$ и, следовательно, имеет более простое детерминантное представление и удовлетворяет более простой иерархии уравнений. Действительно, (5.25) теперь имеет вид

$$
\hat{\tau}_{1}(\xi, \bar{\xi} \mid g) \equiv\left\langle 0_{F_{1}}|\widehat{U}(\xi) g \widehat{\bar{U}}(\bar{\xi})| 0_{F_{1}}\right\rangle=\sum_{k, \bar{k} \geq 0} s_{k} \bar{s}_{\bar{k}}\langle k|g| \bar{k}\rangle,
$$

где $s_{k}=\xi_{1} \xi_{2} \ldots \xi_{k}, s_{0}=1$, a (5.26) заменяется на

$$
\begin{aligned}
& \hat{\tau}_{1}^{m \bar{m}}(\xi, \bar{\xi} \mid g) \equiv\left\langle m_{F_{1}}|\widehat{U}(\xi) g \widehat{\bar{U}}(\bar{\xi})| \bar{m}_{F_{1}}\right\rangle=\frac{1}{s_{m} \bar{s}_{\bar{m}}} \sum_{\substack{k \geq m \\
\bar{k} \geq \bar{m}}} s_{k} \bar{s}_{\bar{k}}\langle k|g| \bar{k}\rangle= \\
& =\frac{1}{s_{m} \bar{s} \bar{m}} \sum_{\substack{k \geq m \\
\bar{k} \geq \bar{m}}} \frac{\partial}{\partial \log s_{k}} \frac{\partial}{\partial \log \bar{s}_{\bar{k}}} \tau_{1}(\xi, \bar{\xi} \mid g)=\frac{1}{s_{m-1} \bar{s}_{\bar{m}-1}} \frac{\partial}{\partial \xi_{m}} \frac{\partial}{\partial \bar{\xi}_{\bar{m}}} \tau_{1}(\xi, \bar{\xi} \mid g) .
\end{aligned}
$$

Поэтому

$$
\begin{aligned}
\hat{\tau}_{n+1} & =\operatorname{det}_{0 \leq m, \bar{m} \leq n} \hat{\tau}_{1}^{m \bar{m}}=\left(\prod_{m=1}^{n} s_{m} \bar{s} \bar{m}\right)^{-1} \operatorname{det}_{(m, \bar{m})}\left(\sum_{\substack{k \geq m \\
\bar{k} \geq \bar{m}}} s_{k} \bar{s}_{\bar{k}}\langle k|g| \bar{k}\rangle\right)= \\
& =\frac{1}{s_{n} \bar{s}_{n}} \sum_{k, \bar{k} \geq n} s_{k} \bar{s}_{\bar{k}} \operatorname{det}_{0 \leq m, \bar{m} \leq n-1}\left(\begin{array}{cc}
g_{m \bar{m}} & g_{m \bar{k}} \\
g_{k \bar{m}} & g_{m \bar{m}}
\end{array}\right) \equiv \\
& \equiv \frac{1}{s_{n} \bar{s}_{n}} \sum_{k, \bar{k} \geq n} s_{k} \bar{s}_{\bar{k}} \mathcal{D}_{k \bar{k}}^{(n)} .
\end{aligned}
$$


Теперь можно сравнить детерминантные представления $(2.24),(2.25)$ и (5.37), чтобы получить связь между различными координатами $t$ и $\xi$. Эта связь имеет структуру ${ }^{20)}$ $s_{k}$ подобно некоторым функциям $P_{j}(t)$.

Столь же просто получить дифференциальные уравнения, которым удовлетворяет $\tau$-функция в параметризации А. Действительно, можно просто найти ФБА (2.41) и подставить их в уравнения (2.42):

$$
\begin{gathered}
\Psi_{n}^{+, n+k+1}(\xi)=\frac{s_{n+k}}{s_{n}}\left(\tau_{n}(\xi)-\xi_{n} \frac{\partial \tau_{n}(\xi)}{\partial \xi_{n}}\right), \\
\Psi_{n}^{+, n+1}(\xi)=\left(\tau_{n}(\xi)-\xi_{n} \frac{\partial \tau_{n}(\xi)}{\partial \xi_{n}}\right), \quad \Psi_{n}^{+, n}(\xi)=-\frac{\partial \tau_{n}(\xi)}{\partial \xi_{n}}, \\
\Psi_{n}^{-, k}(\xi)-\xi_{n-1} \frac{\partial \Psi_{n}^{-, k}(\xi)}{\xi_{n-1}}=\frac{s_{n-1}}{s_{k-1}} \frac{\partial \tau_{n}(\xi)}{\log \xi_{k}}+\frac{s_{n-1}}{s_{k-2}} \frac{\partial \tau_{n}(\xi)}{\partial \xi_{k-1}} \text { для } k>n, \\
\Psi_{n}^{-, n}(\xi)-\xi_{n-1} \frac{\partial \Psi_{n}^{-}, n(\xi)}{\xi_{n-1}}=\tau_{n}(\xi)+\frac{\partial \tau_{n}(\xi)}{\log \xi_{n}}, \\
\Psi_{n}^{-, n-1}(\xi)=\xi_{n-1} \tau_{n}(\xi), \quad \Psi_{n}^{-, k}(\xi)=0 \text { для } k<n-1 .
\end{gathered}
$$

Что касается значений $\Psi_{n}^{+, k}(\xi)$ при $k<n$, то это константы, которые едва ли могут быть представлены действием некоторого дифференциального оператора на $\tau_{n}(\xi)$. Это означает, что соотношение (2.42) (где явные выражения для $\bar{\Psi}$, аналогичные (5.38), также нетрудно выписать) не ведет к дифференциальным уравнениям при произвольно выбранных $k$ и $l$. Однако, если выбрать $k \leq l-1$, из-за многочисленных сокрашений, возникаюших благодаря формулам (5.38), соотношение (2.42) является почти дифференциальным уравнением. Оно может быть легко преобразовано к дифференциальному, если положить $\xi_{n-1}=0$ (см. (5.38)). При этом нетрудно проверить, что число независимых дифференциальных уравнений, полученных таким образом, достаточно для того, чтобы полностью задать $\tau$-функцию. Это означает, что полная иерархия по-прежнему может быть представлена в дифференциальной форме.

\section{4. Квантовый случай $(q \neq 1)$}

Представления $q$-детерминантного типа. Продемонстрируем теперь, как вычисления, описанные в предыдушем пункте, обобщаются на квантовый случай, т.е. группу $S L_{q}(p)$, и, в частности, получим представления $q$-детерминантного типа для $\tau$-функций, подобные (5.30). Кроме того, мы покажем, что в параметризации А соотношение (5.36), выражаюшее $\tau_{1}^{m \bar{m}}$ через производные от $\tau_{1}$, все еше корректно для $q \neq 1$, если заменить производные разностными операторами.

Для получения представлений $q$-детерминантного типа достаточно изучить любой

20) Так, в простейшем случае первого фундаментального представления нужно отождествить $\tau_{1}(t \mid g)=\hat{\tau}_{1}(\xi \mid g)$, т.е.

$$
s_{k}=P_{k}(t), \quad \frac{\partial}{\partial t_{k}}=\sum_{i} s_{i-k} \frac{\partial}{\partial s_{i}} .
$$

Отождествление $\tau_{n}(t)$ и $\hat{\tau}_{n}(\xi)$ с $n \neq 1$ приводит, однако, к другим соотношениям между $\xi$ и $t$. 
$U(\xi)$ вида (5.14), не обрашаясь к конкретной параметризации, подобно тому как это было в случае классической группы ${ }^{21)}$.

Если сравнить со случаем классических групп при $q \neq 1$, то как результат отсутствия диагонального фактора $g_{D}$ копроизведение (5.29) меняет свой вид на

$$
\Delta^{n-1}\left(U\left\{T_{i}\right\}\right)=\prod_{m=1}^{n} U^{(m)}
$$

где

$$
U^{(m)}=U\left\{I \otimes \cdots \otimes I \otimes T_{i} \otimes q^{-2 H_{i}} \otimes \cdots \otimes q^{-2 H_{i}}\right\}
$$

( $T_{i}$ появляется на $m$-м месте в тензорном произведении). Подобным образом получаем

$$
\bar{U}^{(m)}=\bar{U}\left\{q^{2 H_{i}} \otimes \cdots \otimes q^{2 H_{i}} \otimes T_{-i} \otimes I \otimes \cdots \otimes I\right\} .
$$

Положим

$$
H_{i}\left|\bar{j}_{F_{1}}\right\rangle=h_{i, \bar{j}}\left|\bar{j}_{F_{1}}\right\rangle, \quad\left\langle j_{F_{1}}\right| H_{i}=h_{i, j}\left\langle j_{F_{1}}\right|
$$

(в действительности для $S L(p)$ имеем $2 h_{i, i-1}=+1,2 h_{i, i}=-1$, а все остальные равны нулю). Тогда

$$
\begin{gathered}
\tau_{n}^{j_{1} \ldots j_{n} \bar{j}_{1} \ldots \bar{j}_{n}}\left(\xi_{s}, \bar{\xi}_{s} \mid g\right) \equiv\left(\bigotimes_{m=1}^{n}\left\langle j_{m}\right|\right) \Delta^{n-1}(U) g^{\otimes n} \Delta^{n-1}(\bar{U})\left(\bigotimes_{m=1}^{n}\left|\bar{j}_{m}\right\rangle\right)= \\
=\prod_{m=1}^{n}\left\langle j_{m}\left|U\left\{T_{i} q^{-2 \sum_{l=m+1}^{n} h_{i, j_{l}}}\right\} g \bar{U}\left\{T_{-i} q^{2 \sum_{l=1}^{m-1} h_{i, \bar{j}_{l}}}\right\}\right| \bar{j}_{m}\right\rangle= \\
=\prod_{m=1}^{n} \tau_{1}^{j_{m} \bar{j}_{m}}\left(\xi_{s} q^{\left.-2 \sum_{l=m+1}^{n} h_{i(s), j_{l}}, \bar{\xi}_{s} q^{2 \sum_{l=1}^{m-1} h_{i(s), \bar{j}_{l}}}\right) .}\right.
\end{gathered}
$$

Для того чтобы получить $q$-детерминантный аналог (5.30), нужно заменить антисимметризацию $q$-антисимметризацией в уравнениях $(5.27),(5.28)$, поскольку в квантовом случае фундаментальные представления описываются $q$-антисимметризованными векторами (подробности см. в [35, гл. 5.2] и в приложении 2). $q$-Антисимметризация определяется как сумма по всем перестановкам

$$
\left([1, \ldots, k]_{q}\right)=\sum_{P}(-q)^{\operatorname{deg} P}(P(1), \ldots, P(k)),
$$

где

$$
\operatorname{deg} P=\text { количество инверсий в } P \text {. }
$$

Теперь, $q$-антисимметризуя (5.42) с $j_{k}=k-1, \bar{j}_{\bar{k}}=\bar{k}-1$, мы приходим к окончательному ответу

$$
\begin{aligned}
\tau_{n}(\xi, \bar{\xi} \mid g)= & \sum_{P, P^{\prime}}(-q)^{\operatorname{deg} P+\operatorname{deg} P^{\prime}} \prod_{m=0}^{n-1} \tau_{1}^{P(m) P^{\prime}(\bar{m})} \times \\
& \times\left(\xi_{s} q^{-2 \sum_{l=m+1}^{n-1} h_{i(s), P(l)}}, \bar{\xi}_{s} q^{\left.2 \sum_{\bar{l}=0}^{m-1} h_{i(s), P^{\prime}(\bar{l})}\right) .}\right.
\end{aligned}
$$

\footnotetext{
${ }^{21)}$ На самом деле все, что требуется, - это чтобы $U(\xi)$ был элементом из $N \mathbf{G}_{q}$ и выражался только через генераторы, ассоциированные с простыми положительныцми корнями: $U(\xi)=$ $U\left\{\xi_{s} \mid T_{i}\right\}$. Формула (5.14) - возможная, но не единственная реализация этих требований.
} 
Не будь в этом выражении $q$-факторов, “подкручиваюших" времена, это был бы в точности $q$-детерминант, определяемый формулой

$$
\operatorname{det}_{q} A \sim A_{[1}^{[1} \ldots A_{n]_{q}}^{n]_{q}}=\sum_{P, P^{\prime}}(-q)^{\operatorname{deg} P+\operatorname{deg} P^{\prime}} \prod_{a} A_{P^{\prime}(a)}^{P(a)} .
$$

Конкретный пример формулы (5.45) в простейшем содержательном случае второго фундаментального представления, иллюстрируюший его структуру, рассмотрен в приложении 3.

Заметим, что $q$-факторы во всех полученных выше выражениях можно тривиально воспроизводить действием операторов

$$
\begin{array}{ll}
M_{j}^{ \pm}: & M_{j}^{ \pm} \xi_{s}=q^{ \pm \delta_{j, i(s)}} \xi_{s}, \\
\bar{M}_{j}^{ \pm}: & \bar{M}_{j}^{ \pm} \bar{\xi}_{s}=q^{ \pm \delta_{j, i(s)}} \bar{\xi}_{s} .
\end{array}
$$

Мы уже неоднократно видели, что их наличие типично для деформаций интегрируемых иерархий.

Скажем теперь несколько слов об уравнениях, которым удовлетворяет квантовая $\tau$-функция. Для их вывода нужно действовать аналогично классическому случаю и ввести сплетаюшие операторы. В квантовом случае необходимо различать правые и левые сплетаюшие операторы: $\Phi^{ \pm, R}: F_{n} \otimes F_{1} \rightleftarrows F_{n+1}$ и $\Phi^{ \pm, L}: F_{1} \otimes F_{n} \rightleftarrows F_{n+1}$. Эти операторы $\Phi^{ \pm, R, L}$ могут быть выражены через классические сплетаюшие операторы

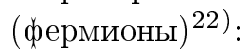

$$
\Phi_{i}^{ \pm, R}=q^{-\sum_{j=1}^{i-1} \psi_{j}^{+} \psi_{j}^{-}} \psi_{i}^{ \pm}, \quad \Phi_{i}^{ \pm, L}=q^{\sum_{j=1}^{i-1} \psi_{j}^{+} \psi_{j}^{-}} \psi_{i}^{ \pm} .
$$

Далее, по аналогии с классическим случаем надо рассмотреть оператор $\Gamma=\sum_{i} \Phi_{i}^{+, L} \otimes$ $\Phi_{i}^{-, R}$, коммутируюший с $g \otimes g$. Теперь, вводя квантовые ФБА как среднее квантовых сплетаюших операторов $\Phi^{ \pm}$(с подходящими индексами $L$ и $R$ ) и соответствуюшие им вершинные операторы, мы получим те же уравнения (2.39)-(5.32), но с переопределенными величинами. Технически действие вершинного оператора может быть вычислено с помошью формулы (5.47), что приводит к квантовому аналогу формул (2.31), (2.32).

Фиксируем теперь конкретную параметризацию А и увидим, как все эти формулы работают.

Параметризация А. В этой параметризации большинство выражений, полученных выше, остается практически без изменений, хотя в квантовом случае, естественно, следует позаботиться об упорядочении различных объектов. В частности, имеем

$$
\hat{\tau}_{1}(\xi, \bar{\xi} \mid g) \equiv\left\langle 0_{F_{1}}|\widehat{U}(\xi) g \widehat{\bar{U}}(\bar{\xi})| 0_{F_{1}}\right\rangle=\sum_{k, \bar{k} \geq 0} s_{k} \bar{s}_{\bar{k}}\langle k|g| \bar{k}\rangle
$$

22) Это следствие похожей структуры неприводимых представлений квантовых и классических УОА при $|q| \neq 1$ [98]. 
где опять $s_{k}=\xi_{1} \xi_{2} \ldots \xi_{k}, s_{0}=1$, но $\bar{s}_{k}=\bar{\xi}_{k} \ldots \bar{\xi}_{2} \bar{\xi}_{1}, \bar{s}_{0}=1$, при этом

$$
\begin{aligned}
\hat{\tau}_{1}^{m \bar{m}}(\xi, \bar{\xi} \mid g) & =s_{m}^{-1}\left(\sum_{\substack{k \geq m \\
\bar{k} \geq \bar{m}}} s_{k} \bar{s}_{\bar{k}}\langle k|g| \bar{k}\rangle\right) \bar{s}_{\bar{m}}^{-1}= \\
& =s_{m-1}^{-1}\left(D_{\xi_{m}} \bar{D}_{\bar{\xi}_{\bar{m}}} \tau_{1}(\xi, \bar{\xi} \mid g)\right) \bar{s}_{\bar{m}-1}^{-1} .
\end{aligned}
$$

Здесь ${ }^{23)}$

$$
D_{\xi_{i}} f(\xi) \equiv \frac{1}{\xi_{i}} \frac{M_{i}^{+2}-1}{q^{2}-1} f(\xi), \quad \bar{D}_{\bar{\xi}_{i}} f(\bar{\xi}) \equiv\left[\frac{M_{i}^{-2}-1}{q^{-2}-1} f(\bar{\xi})\right] \frac{1}{\bar{\xi}_{i}}
$$

(в этих операторах порядок величин важен!). Теперь можно явно выразить $\tau_{n}$ через $\tau_{1}$, используя формулы (5.45) и (5.49). Как все это выглядит в простейшем примере второго фундаментального представления, описано в приложении 3.

ФБА для $\tau$-функции в параметризации А дается следуюшими выражениями:

$$
\begin{gathered}
\Psi_{n}^{+, n+k+1}(\xi)=q^{n+1} s_{n}^{-1} s_{n+k} M_{n+1}^{+} \ldots M_{n+k-1}^{+}\left(\tau_{n}(\xi)-\xi_{n} D_{n} \tau_{n}(\xi)\right), \\
\Psi_{n}^{+, n+1}(\xi)=q^{n+1}\left(\tau_{n}(\xi)-\xi_{n} D_{n} \tau_{n}(\xi)\right), \quad \Psi_{n}^{+, n}(\xi)=-q^{n} D_{n} \tau_{n}(\xi), \\
\Psi_{n}^{-, k}(\xi)-\xi_{n-1} D_{n-1} \Psi_{n}^{-, k}(\xi)= \\
=q^{n-2} s_{k-2}^{-1} s_{n-1} D_{i-1} \tau_{n}(\xi)+q^{n-2} s_{k-1}^{-1} s_{n-1} \xi_{i} D_{i} \tau_{n}(\xi) \quad \text { для } \quad k>n, \\
\Psi_{n}^{-, n}(\xi)-\xi_{n-1} D_{n-1} \Psi_{n}^{-,, n}(\xi)=q^{n-2} \tau_{n}(\xi)+\xi_{n} D_{n} \tau_{n}(\xi), \\
\Psi_{n}^{-, n-1}(\xi)=q^{n-2} \xi_{n-1} \tau_{n}(\xi), \quad \Psi_{n}^{-, k}(\xi)=0 \quad \text { для } \quad k<n-1 .
\end{gathered}
$$

Подставляя эти формулы в (2.42), мы получаем набор уравнений, являюшихся квантовым аналогом иерархии КП/Тоды в параметризации А.

В заключение данного раздела отметим еше раз, что детерминантные представления для классических $\tau$-функций не сводятся в точности к $q$-детерминантным при $q \neq 1$. Причиной этого было то, что оператор эволюции в квантовом случае не был групповым элементом. Этот факт, в свою очередь, связан с отсутствием у квантовой группы нильпотентных подгрупп $N \mathbf{G}_{q}$. Чтобы избежать этой проблемы, можно начать со слегка иной параметризации $\tau$-функции такой, что оператор эволюции лежит не в нильпотентной, а в борелевской подгруппе $B \mathbf{G}_{q}$. При этом в классическом пределе добавочные картановские генераторы могут быть просто убраны переопределением элемента $g$ (задаюшего точку грассманниана). Однако в квантовом случае картановская часть оператора эволюции сушественно меняет результат - эволюционный оператор становится групповым элементом (для $B \mathbf{G}_{q}$ ) и все "подкрутки" времен в формуле (5.45) исчезают. Определенная таким образом $\tau$-функция является просто $q$-детерминантом. Для полного решения этой схемы все еше нужно найти подходящую параметризацию (набора) групповых элементов $B \mathbf{G}_{q}$ в точности $r_{\mathbf{G}}$ "временами".

\footnotetext{
23) Существует некоторая неоднозначность в выборе этих операторов, поскольку $\tau$-функция линейна по временам, и, следовательно, подходит любой линейный оператор, делающий 1 из $\xi$. Наш выбор разностных операторов обусловлен естественностью их действия на $q$-экспоненту и соответствием с разностными операторами, используемыми на всем протяжении обзора.
} 


\section{6. ВОЛНОВАЯ ФУНКЦИЯ И $S$-МАТРИЦА В КВАНТОВОЙ ТЕОРИИ ЛИУВИЛЛЯ (ТОДЫ)}

Как мы видели выше, группа, действуюшая в пространстве спектрального параметpa, т.е. на решениях интегрируемой системы, при квантовании должна быть просто заменена квантовой группой, фактически это и составляет смысл процедуры квантования. Теперь мы рассмотрим пример другой групповой структуры, которая также может быть выявлена в теории Тоды. Речь идет о группе, которая действует в "пространстве-времени", т.е. на переменных уравнения. Замечательным свойством этой группы является то, что при квантовании она не меняется, однако меняются изучаемые объекты и способ работы с данной групповой структурой.

А именно, рассмотрим опять молекулу Тоды, дополнительно редуцированную к цепочке Тоды, т.е. зависяшую только от суммы времен. Покажем, что эта система получается гамильтоновой редукцией свободной системы, заданной на кокасательном расслоении к группе Ли [41-43, 99].

\section{1. Классическая система Тоды как гамильтонова редукция}

Действительно, рассмотрим кокасательное расслоение $T^{*} \mathbf{G}$ для вещественной расшепимой группы G. При помоши групповых сдвигов оно может быть сведено к паре $(Y, g), Y \in \mathcal{G}^{*}, g \in \mathbf{G}$, где $\mathcal{G}$ - соответствуюшая группе $\mathbf{G}$ алгебра. На $T^{*} \mathbf{G}$ сушествуют каноническая биинвариантная симплектическая форма

$$
\omega=\delta Y\left(\delta g g^{-1}\right)
$$

и набор инвариантных коммутируюших гамильтонианов

$$
\frac{\left\langle Y^{d_{k}}\right\rangle}{d_{k}}, \quad k=1, \ldots, r
$$

где $d_{k}=2, \ldots-$ инварианты $\mathcal{G}$, а $Y^{d_{k}}-$ ad $^{*} \mathbf{G}$-инвариантные полиномы на $\mathcal{G}^{*}$. Это задает свободную гамильтонову систему.

Рассмотрим теперь симплектическую редукцию $T^{*} \mathbf{G}$ (т.е. редукцию, сохраняюшую симплектическую форму $\omega(6.1))$ относительно следуюшего действия: $\bar{N} \oplus N$ - левой и правой нильпотентных подгрупп группы $\mathbf{G}$ :

$$
\begin{array}{lll}
g \mapsto v g, & Y \mapsto v Y v^{-1}, & v \in \bar{N}, \\
g \mapsto g n, & Y \mapsto Y, & n \in N .
\end{array}
$$

Это действие задает два отображения моментов

$$
\mu_{v}=\operatorname{Pr}_{\overline{\mathcal{N}}^{*}} Y, \quad \mu_{n}=\operatorname{Pr}_{\mathcal{N}^{*}} g^{-1} Y g .
$$

Используя разложение Гаусса, можно преобразовать $g$ при помощи (6.3) к картановской подгруппе $A$. Пусть $g=\exp \phi, \phi \in \mathcal{A}$. Предположим, что

$$
\mu_{v}=\operatorname{Pr}_{\overline{\mathcal{N}}^{*}} Y=\mu^{L}, \quad \mu_{n}=\operatorname{Pr}_{\mathcal{N}^{*}} g^{-1} Y g=\mu^{R},
$$


где

$$
\mu^{L}=\sum_{\alpha \in \Pi} \mu_{\alpha}^{L} \mathcal{G}_{\alpha}, \quad \mu^{R}=\sum_{\alpha \in \Pi} \mu_{\alpha}^{R} \mathcal{G}_{-\alpha},
$$

$\mu_{\alpha}^{R, L}$ - произвольные константы и П обозначает набор всех простых положительных корней. После “диагонализации" $g$ разложением Гаусса в точке $g=\exp \phi, \phi \in \mathcal{A}=h_{\mathbf{G}}$, мы можем явно разрешить условие связи (6.5):

$$
Y=p+\sum_{\alpha \in \Pi}\left(\mu_{\alpha}^{R} e^{\alpha(\phi)} \mathcal{G}_{\alpha}+\mu_{\alpha}^{L} \mathcal{G}_{-\alpha}\right) .
$$

При этом редуцированная симплектическая форма принимает канонический вид

$$
\omega^{\mathrm{red}}=\delta p \delta \phi=\sum_{k=1}^{r} \delta p_{k} \delta \phi_{k},
$$

а редуцированное фазовое пространство $\bar{N} \backslash \backslash T^{*} \mathbf{G} / / N$ имеет размерность

$$
2 \operatorname{dim} \mathbf{G}-2 \operatorname{dim} \bar{N}-2 \operatorname{dim} N=2 r .
$$

Комбинация

$$
W\left(Y ; \tau_{1}, \ldots, \tau_{r}\right)=\sum_{k=1}^{r} \frac{\tau_{k}}{d_{k}}\left\langle Y^{d_{k}}\right\rangle
$$

определяет коммутируюшие гамильтонианы классической иерархии, заданной оператором Лакса (6.6). В частности,

$$
\left\langle Y^{2}\right\rangle=\frac{1}{2} \sum_{k=1}^{r} p_{k}^{2}+\sum_{\alpha \in \Pi} \mu_{\alpha} e^{2 \alpha(\phi)}
$$

- стандартный гамильтониан цепочки Тоды [100]. Классическое действие в этом представлении приобретает вид

$$
S^{I}=\int Y\left(\partial_{t} g g^{-1}\right)-\left\langle Y^{2}\right\rangle+\left\langle B_{L}, Y-\mu^{L}\right\rangle+\left\langle B_{R}, g^{-1} Y g-\mu^{R}\right\rangle,
$$

где $B_{L} \in \overline{\mathcal{N}}, B_{R} \in \mathcal{N}$ - лагранжевы множители.

Для сравнения со стандартными формулами цепочки Тоды достаточно выбрать в качестве $\mathbf{G}$ группу $S L(p)$ и рассмотреть ее фундаментальноепредставление. Тогда $Y(6.6)$ совпадает с оператором Лакса цепочки Тоды и т.д.

Построенная выше конструкция сушественно основывалась на разложении Гаусса [39]. В действительности к той же системе можно прийти и с помошью разложения Ивасавы. Именно эта последняя является стандартной, до сих пор применявшейся для исследования подобных систем. В частности, в следующих пунктах мы рассмотрим квантование системы Тоды, которое было проведено в рамках именно разложения Ивасавы [51] и которое приводит к так называемой волновой функции (В $\Phi)$ Уиттекера. Мы, однако, всюду будем пользоваться разложением Гаусса, более удобным для применения к афффинным алгебрам [39]. Подробности и обсуждение связи результатов, полученных в рамках двух разложений - Гаусса и Ивасавы, можно найти в [39]. 


\section{2. Квантовая механика Лиувилля, общая схема решения}

Нетривиальным свойством обнаруженной выше “пространственно-временной” группы $^{24)} S L(p)$-молекулы Тоды является то, что при квантовании эта группа остается классической, меняется только ее трактовка - квантование вводит орбитную интерпретацию. Действительно, если классическая система получается гамильтоновой редукцией свободной системы, заданной на кокасательном расслоении к простой вещественной группе Ли [41-43], то квантовая модель связана с неприводимыми унитарными представлениями той же самой группы. Таким образом, квантовая система должна скорее трактоваться в рамках геометрического квантования [44].

Опишем вкратце общий подход, который позволяет решать дифференциальные уравнения на собственные значения (С3) и, в частности уравнения Шредингера, в групповых терминах, а затем рассмотрим конкретные примеры. Технически необходимо сделать несколько шагов.

1. Пусть $g(\xi \mid T) \in A_{\mathbf{G}}(\xi) \otimes U_{\mathcal{G}}$ - “универсальный групповой элемент" (см. пункт 4.3) группы Ли $\mathbf{G}^{25)}$, где $\xi$ некоторым образом параметризует групповое многообразие, а $T$ - генераторы $\mathbf{G}$ в некотором (не обязательно неприводимом) представлении, причем их единственным свойством являются коммутационные соотношения $\left[T^{a}, T^{b}\right]=f^{a b c} T^{c}$.

2. Для каждой данной параметризации $\{\xi\}$ можно ввести два набора дифференциальных операторов $\mathcal{D}_{R, L}(\xi)$ таких, что

$$
\begin{aligned}
& \mathcal{D}_{L}^{a}(\xi) g(\xi \mid T)=T^{a} g(\xi \mid T), \\
& \mathcal{D}_{R}^{a}(\xi) g(\xi \mid T)=g(\xi \mid T) T^{a} .
\end{aligned}
$$

Эти операторы удовлетворяют очевидным коммутационным соотношениям

$$
\begin{gathered}
{\left[\mathcal{D}_{L}^{a}, \mathcal{D}_{L}^{b}\right]=-f^{a b c} \mathcal{D}_{L}^{c},} \\
{\left[\mathcal{D}_{R}^{a}, \mathcal{D}_{R}^{b}\right]=f^{a b c} \mathcal{D}_{R}^{c},} \\
{\left[\mathcal{D}_{L}^{a}, \mathcal{D}_{R}^{b}\right]=0 .}
\end{gathered}
$$

3. Для данного представления $\mathcal{R}$, скалярного произведения $\langle\mid\rangle$ и двух элементов представления $\left\langle\psi_{L}\right|$ и $\left|\psi_{R}\right\rangle$ построим матричный элемент

$$
F_{\mathcal{R}}\left(\xi \mid \psi_{L}, \psi_{R}\right)=\left\langle\psi_{L}|g(\xi \mid T)| \psi_{R}\right\rangle .
$$

Действие любой комбинации дифференциальных операторов $\mathcal{D}_{R}$ на $F$ эквивалентно вставке той же комбинации генераторов $T$ справа от $g(\xi \mid T)$. Если $\left|\psi_{R}\right\rangle$ является собственным вектором этой комбинации генераторов, то $F$ дает решение соответствующего дифференциального уравнения (в форме задачи на СЗ).

\footnotetext{
24) Эта пространственно-временная группа не должна смешиваться с группой $S L(p)$, описываюшей редукцию к молекуле Тоды (см. раздел 3 ). Чтобы понять, что это две совершенно различные группы, достаточно заметить, что двумеризованная молекула Тоды описывается той же группой редукции $S L(p)$, но афффинной пространственно-временной группой $\widehat{S} L(p)$.

$25)$ В качестве этой группы можно выбрать и квантовую группу, однако это ведет к разностному уравнению Лиувилля, см. [101].
} 
4. Особый интерес представляют операторы Казимира, поскольку $\left|\psi_{R}\right\rangle$, являющиеся их собственными векторами, описываются просто как элементы неприводимых представлений.

5. В частности, квадратичный оператор Казимира, выраженный через $\mathcal{D}_{R}$, является оператором Лапласа и приводит к некоторым важным уравнениям Шредингера, типичным примером которых является уравнение для системы типа Тоды.

На самом деле для получения уравнения Шредингера системы типа Тоды необходимо наложить дополнительные ограничения на состояния $\left\langle\psi_{L}\right|$ и $\left|\psi_{R}\right\rangle$, соответствуюшие гамильтоновой редукции (6.3) свободного движения на групповом многообразии группы $\mathbf{G}$ в классическом случае. Такая редукция, как мы видели в предыдущем пункте, ассоциирована с некоторым разложением $\mathbf{G}$ в произведение подгрупп, причем различные разложения могут приводить к эквивалентным редукциям. По сушеству, редукция приводит к независимости $F(\xi)$ от некоторых координат $\xi$. Для конечномерных групп Ли остаюшиеся координаты могут быть выбраны отвечаюшими картановскому тору, в то время как для аффинных алгебр естественнее сохранить зависимость от всех диагональных матриц. Обсуждаемый здесь матричный элемент - ВФ F - иногда называется функцией Уиттекера.

Заметим, что дифференциальные операторы $\mathcal{D}_{L}$ и $\mathcal{D}_{R}$ реализуют соответственно левое и правое регулярные представления алгебры групшы $\mathbf{G}$ (левое в действительности является антипредставлением), которые инвариантно задаются действием на алгебpe $A_{\mathbf{G}}$ функций на группе:

$$
\pi_{\mathrm{reg}}^{L}(h) f(g)=f(h g), \quad \pi_{\mathrm{reg}}^{R}(h) f(g)=f(g h), \quad g, h \in G .
$$

Явно эти операторы можно получить следующим образом. Рассмотрим групповой элемент $g$ и (формальный) дифференциальный оператор d, действующий как полная производная на функции от $\xi_{i}$, т.е.

$$
\mathrm{d} \equiv \sum_{i} d \xi_{i} \frac{\partial}{\partial \xi_{i}} .
$$

Далее вычислим величину $g^{-1} \cdot \mathrm{d} g$ (форма Маурера-Картана) и разложим ее по генераторам алгебры :

$$
g^{-1} \cdot \mathrm{d} g=\sum_{a, i} c_{a, i} T_{a} d \xi_{i}
$$

т.e.

$$
\mathrm{d} g=\sum_{a, i} c_{a, i}\left(\mathcal{D}_{R}^{a} g\right) d \xi_{i}
$$

Из этого выражения нетрудно найти явные формулы для дифференциальных операторов $\mathcal{D}_{R}$. Оператор $\mathcal{D}_{L}$ вычисляется совершенно аналогично.

Заметим, что достаточно рассмотреть $g$ только в фундаментальном представлении, поскольку матричные элементы фундаментального представления генерируют всю алгебру $A_{\mathrm{G}}$, а действие группы может быть расширено на всю эту алгебру при помоши копроизведения. Вычисления же коэффициентов $c_{a, i}$ в фундаментальном представлении весьма просты.

В качестве примера рассмотренной общей схемы ниже мы кратко изучим пример уравнения Шредингера для системы типа Тоды и опишем решение соответствуюшей 
квантовой системы, отсылая за подробностями к работе [39]. Начнем с простейшего случая группы $S L(2)$, т.е. лиувиллевской квантовой механики. Задача, которую мы будем решать, - найти интегральное представление для В $\Phi$ и ее асимптотики, определяющие $S$-матрицу рассеяния.

\section{3. Квантовая механика Лиувилля, группа $S L(2, \mathbf{R})$}

Система Лиувилля для группы $S L(2, \mathbf{R})$. Соответствуюшая алгебра Ли задается соотношениями

$$
\left[T_{+}, T_{-}\right]=T_{0}, \quad\left[T_{ \pm}, T_{0}\right]=\mp 2 T_{ \pm} .
$$

В фундаментальном представлении

$$
T_{0}=\left(\begin{array}{cc}
1 & 0 \\
0 & -1
\end{array}\right), \quad T_{+}=\left(\begin{array}{ll}
0 & 1 \\
0 & 0
\end{array}\right), \quad T_{-}=\left(\begin{array}{ll}
0 & 0 \\
1 & 0
\end{array}\right) .
$$

Квадратичный оператор Казимира равен

$$
C=\left(T_{-} T_{+}+T_{+} T_{-}\right)+\frac{1}{2} T_{0}^{2}=2 T_{-} T_{+}+T_{0}+\frac{1}{2} T_{0}^{2} .
$$

Выполним теперь первый шаг нашей обшей конструкции - параметризуем групповой элемент следуюшим образом ${ }^{26)}$ :

$$
g(\psi, \phi, \chi \mid T)=e^{\psi T_{-}} e^{\phi T_{0}} e^{\chi T_{+}} .
$$

В фундаментальном представлении групповой элемент имеет вид

$$
g=\left(\begin{array}{ll}
1 & 0 \\
\psi & 1
\end{array}\right)\left(\begin{array}{cc}
e^{\phi} & 0 \\
0 & e^{-\phi}
\end{array}\right)\left(\begin{array}{cc}
1 & \chi \\
0 & 1
\end{array}\right)=\left(\begin{array}{cc}
e^{\phi} & \chi e^{\phi} \\
\psi e^{\phi} & \psi \chi e^{\phi}+e^{-\phi}
\end{array}\right) .
$$

В качестве второго шага мы должны построить дифференциальные операторы правого и левого регулярных представлений. Как уже говорилось выше, для этого нужно вычислить “токи" $g^{-1} \cdot \mathrm{d} g$ и $\mathrm{d} g \cdot g^{-1}$ :

$$
\begin{aligned}
g^{-1} \cdot \mathrm{d} g & =\left(\begin{array}{cc}
-e^{2 \phi} \chi d \psi+d \phi & -e^{2 \phi} \chi^{2} d \psi+2 \chi d \phi+d \chi \\
e^{2 \phi} d \psi & e^{2 \phi} \chi d \psi-d \phi
\end{array}\right), \\
\mathrm{d} g \cdot g^{-1} & =\left(\begin{array}{cc}
-e^{2 \phi} \psi d \chi+d \phi & e^{2 \phi} d \chi \\
-e^{2 \phi} \psi^{2} d \chi+2 \psi d \phi+d \psi & e^{2 \phi} \psi d \chi-d \phi
\end{array}\right) .
\end{aligned}
$$

Отсюда, используя (6.15), легко получаем

$$
\begin{aligned}
& \frac{\partial g}{\partial \phi}=g\left(T^{0}+2 \chi T^{+}\right)=\left(T^{0}+2 \psi T^{-}\right) g \\
& \frac{\partial g}{\partial \chi}=g T^{+}=e^{2 \phi}\left(T^{+}-\psi T^{0}-\psi^{2} T^{-}\right) g \\
& \frac{\partial g}{\partial \psi}=g\left(-\chi^{2} T^{+}-\chi T^{0}+T^{-}\right) e^{2 \phi}=T^{-} g
\end{aligned}
$$

26) Этот способ отличается от принятого в предыдущих разделах перестановкой нильпотентных подгрупп, которая явно задается действием внутреннего автоморфизма группы - антипода. 
а, используя (6.16), -

$$
\begin{array}{lll}
\mathcal{D}_{R}^{+}=\frac{\partial}{\partial \chi}, & \mathcal{D}_{R}^{0}=-2 \chi \frac{\partial}{\partial \chi}+\frac{\partial}{\partial \phi}, & \mathcal{D}_{R}^{-}=e^{-2 \phi} \frac{\partial}{\partial \psi}+\chi \frac{\partial}{\partial \phi}-\chi^{2} \frac{\partial}{\partial \chi} \\
\mathcal{D}_{L}^{-}=\frac{\partial}{\partial \psi}, & \mathcal{D}_{L}^{0}=-2 \psi \frac{\partial}{\partial \psi}+\frac{\partial}{\partial \phi}, & \mathcal{D}_{L}^{+}=-\psi^{2} \frac{\partial}{\partial \psi}+\psi \frac{\partial}{\partial \phi}+e^{-2 \phi} \frac{\partial}{\partial \chi}
\end{array}
$$

На третьем шаге мы должны выбрать представление. Рассмотрим главную (сферическую) серию представлений, индуцированную одномерными представлениями борелевской подалгебры [102]. Пространством представления являются функции одной переменной, а матричные элементы (скалярное произведение) задаются интегралом с плоской мерой. Действие алгебры определяется дифференциальными операторами

$$
T_{+}=\frac{\partial}{\partial x}, \quad T_{0}=-2 x \frac{\partial}{\partial x}+2 j, \quad T_{-}=-x^{2} \frac{\partial}{\partial x}+2 j x,
$$

где $j$ - спин представления. Мы будем рассматривать только унитарные представления, поскольку только для них скалярное произведение задается сходяшимся интегралом. $\mathrm{y}_{\text {словие унитарности означает, что } j+1 / 2 \text { является чисто мнимым }}{ }^{27)}$.

В качестве четвертого шага рассмотрим квадратичный оператор Казимира (его можно вычислять как в левом, так и в правом регулярных представлениях):

$$
C=\frac{1}{2} \frac{\partial^{2}}{\partial \phi^{2}}+\frac{\partial}{\partial \phi}+2 e^{-2 \phi} \frac{\partial^{2}}{\partial \psi \partial \chi} .
$$

Его СЗ в представлении спина $j$ равно $2\left(j^{2}+j\right)$. Следовательно, матричньй элемент $F=\left\langle\psi_{L}|g(\theta, \phi, \chi)| \psi_{R}\right\rangle$ с $\left\langle\psi_{L}\right|$ и $\left\langle\psi_{R}\right|$, принадлежашими представлению спина $j$, удовлетворяет уравнению

$$
C F=2\left(j^{2}+j\right) F
$$

Теперь в качестве последнего, пятого шага мы должны задать условия редукции. Выберем их в виде

$$
\frac{\partial}{\partial \chi} F_{G}=i \mu_{R} F_{G}, \quad \frac{\partial}{\partial \psi} F_{G}=i \mu_{L} F_{G}
$$

т.е. (см. $(6.24)$ и $(6.25))$

$$
T_{+}\left|\psi_{R}\right\rangle=i \mu_{R}\left|\psi_{R}\right\rangle, \quad\left\langle\psi_{L}\right| T_{-}=i \mu_{L}\left\langle\psi_{L}\right| .
$$

При такой редукции гамильтониан (квадратичный оператор Казимира) равен (ср. (6.9))

$$
H=\frac{1}{2} \frac{\partial^{2}}{\partial \phi^{2}}+\frac{\partial}{\partial \phi}-2 \mu_{R} \mu_{L} e^{-2 \phi},
$$

а функция $\Psi(\phi)=e^{\phi} F$ удовлетворяет следуюшему уравнению Шредингера:

$$
\left[\frac{1}{2} \frac{\partial^{2}}{\partial \phi^{2}}-2 \mu_{R} \mu_{L} e^{-2 \phi}\right] \Psi(\phi)=2\left(j+\frac{1}{2}\right)^{2} \Psi(\phi) \equiv \lambda^{2} \Psi(\phi),
$$

\footnotetext{
${ }^{27)}$ Аналогично в случае общей $S L(p)$ мнимыми должны быть все $j_{i}+1 / 2$, т.е. $\mathbf{j}+\boldsymbol{\rho}$
} 
которое, действительно, является уравнением Шредингера для системы Лиувилля.

Заметим, что, используя представление (6.21) и условия (6.30), мы можем непосредственно получить уравнение (6.31) из следуюшей цепочки равенств ${ }^{28)}$ :

$$
\begin{aligned}
2 j(j+1) F_{G}^{(j)} & \equiv 2 j(j+1)\left\langle\psi_{L}\left|e^{\phi T_{0}}\right| \psi_{R}\right\rangle_{j}=\left\langle\psi_{L}\left|e^{\psi T_{-}} e^{\phi T_{0}} e^{\chi T_{+}} \widehat{C}\right| \psi_{R}\right\rangle_{j}= \\
& =\left\langle\psi_{L}\left|e^{\phi T_{0}}\left(2 T_{-} T_{+}+T_{0}+\frac{1}{2} T_{0}^{2}\right)\right| \psi_{R}\right\rangle_{j}= \\
& =\left(-2 \mu_{R} \mu_{L} e^{-2 \phi}+\frac{\partial}{\partial \phi}+\frac{1}{2} \frac{\partial^{2}}{\partial \phi^{2}}\right)\left\langle\psi_{L}\left|e^{\phi T_{0}}\right| \psi_{R}\right\rangle_{j} .
\end{aligned}
$$

Решение лиувиллевского уравнения Шредингера. Теперь нам остается только решить условия редукции (6.30) и найти явное интегральное представление для ВФ. При этом мы используем формулы (6.26). Тогда условия (6.30) имеют вид

$$
\begin{gathered}
T_{+}\left|\psi_{R}\right\rangle=\frac{\partial}{\partial x} \psi_{R}(x)=i \mu_{R} \psi_{R}(x) \\
\left\langle\psi_{L}\right| T_{-}=\left(2 x+x^{2} \frac{\partial}{\partial x}+2 j x\right) \psi_{L}(x)=i \mu_{L} \psi_{L}(x) .
\end{gathered}
$$

Их решения следуюшие:

$$
\psi_{R}(x)=e^{i \mu_{R} x}, \quad \psi_{L}(x)=x^{-2(j+1)} e^{-\frac{i \mu_{L}}{x}} .
$$

Это дает нам окончательно решение уравнения Шредингера (6.32):

$$
\begin{aligned}
e^{\phi} F^{(j)}(\phi) & =e^{\phi}\left\langle\psi_{L}\left|e^{\phi T_{0}}\right| \psi_{R}\right\rangle_{j}=e^{\phi} \int x^{-2(j+1)} e^{-\frac{i \mu_{L}}{x}} e^{\phi\left(2 j-2 x \frac{\partial}{\partial x}\right)} e^{i \mu_{R} x} d x= \\
& =\left(\frac{i}{\mu_{R}}\right)^{-(2 j+1)} e^{-(2 j+1) \phi} \int_{0}^{\infty} x^{-2(j+1)} e^{-\frac{\mu_{L} \mu_{R} e^{-2 \phi}}{x}-x} d x= \\
& =2\left(i \sqrt{\frac{\mu_{L}}{\mu_{R}}}\right)^{-(2 j+1)} K_{2 j+1}\left(2 \sqrt{\mu_{L} \mu_{R}} e^{-\phi}\right) d x
\end{aligned}
$$

где $K_{\nu}(z)$ - функция Макдональда [103].

Функция Хариш-Чандры и асимптотики. Вернемся еше раз к уравнению Шредингера (6.32). Условие унитарности представления означает чисто мнимые $\lambda \equiv \sqrt{2}(j+$ $1 / 2)$, т.е. непрерывный спектр в уравнении Шредингера (и вешественную энергию). Решения этого уравнения, естественно, задаются осциллируюшей функцией только на одной бесконечности (из-за бесконечно растушего потенщиала), а на другой - экспоненциально спадающей функцией. Мы можем поставить задачу рассеяния. При этом $S$-матрица отражения равна отношению коэффициентов при падающей и отраженной волнах. При этом нам потребуется выделить две экспоненциальные асимптотики $e^{ \pm \lambda \phi}$ на одной и той же бесконечности. Технически это делается следующим образом: рассматривается маленькая вешественная добавка к $\lambda$ и в зависимости от ее знака выделяется одна из двух асимптотик.

\footnotetext{
${ }^{28)} \mathrm{C}$ этого момента мы рассматриваем только матричный элемент от $e^{\phi_{I} T_{0}}$ вместо $g$, для того чтобы исключить тривиальные $\psi$ - и $\chi$-зависимости $F$.
} 
Проделаем эту процедуру с функцией Макдональда:

$$
K_{\nu}(z)=\frac{1}{2}\left(\frac{z}{2}\right)^{\nu} \int_{0}^{\infty} e^{-t-\frac{z^{2}}{4 t}} t^{-\nu-1} d t
$$

Если $\operatorname{Re} \nu \leq 0$, в асимптотике (т.е. при малых $z$ ) можно отбросить член $z^{2} / 4 t$, так что интеграл равен

$$
K_{\nu}(z) \underset{z \rightarrow 0}{\sim} \frac{1}{2}\left(\frac{z}{2}\right)^{\nu} \int_{0}^{\infty} e^{-t} t^{-\nu-1} d t=-\frac{\pi}{2} \frac{1}{\sin \pi \nu \Gamma(1+\nu)}\left(\frac{z}{2}\right)^{\nu} .
$$

$\mathrm{C}$ другой стороны, при $\operatorname{Re} \nu \geq 0$ нужно заменить $t \rightarrow z^{2} t$, в результате получим

$$
K_{\nu}(z) \underset{z \rightarrow 0}{\sim} \frac{1}{2}(2 z)^{-\nu} \int_{0}^{\infty} e^{-\frac{1}{4 t}} t^{-\nu-1} d t=\frac{\pi}{2} \frac{1}{\sin \pi \nu \Gamma(1-\nu)}\left(\frac{z}{2}\right)^{-\nu} .
$$

Заметим, что хотя отношение двух полученных асимптотик решения определено однозначно, в определении каждой из них по отдельности сохраняется произвол, связанный с обшим нормировочным множителем ВФ. Эту нормировку принято частично фиксировать требованием отсутствия полюсов при конечных значениях импульса. Вопрос полной фиксации нормировки обсуждается в [39].

В данном случае отсутствие полюсов означает, что полученную В $(6.36)$ следует домножить на функцию $\sin \pi \lambda$, которая, уничтожая все полюсы, привносит дополнительные нули. Две асимптотики нормированной таким образом ВФ имеют вид ${ }^{29)}$

$$
c_{ \pm}=\frac{1}{\Gamma(1 \pm \lambda)} .
$$

Эти функции называются функциями Хариш-Чандры [104], они играют важную роль в теории групп

\section{4. Квантовая механика Тоды}

Квантовая механика молекулы Тоды, группа $S L(p, R)$. Применим теперь развитую нами обшую конструкцию к несколько более общему случаю - группе $S L(p, R)$. Вычисления при этом вполне аналогичны проделанным в предыдущем пункте. Однако они требуют некоторой дополнительной информации о структуре унитарных представлений $S L(p, R)$, поэтому мы приведем здесь только результаты, сославшись на приложение 4 и, особенно, работу [39], которые содержат подробные вычисления.

А именно, как и в предыдушем пункте, мы должны рассмотреть матричный элемент $F$, заданный в унитарном неприводимом представлении группы и подействовать на него оператором Казимира. Поскольку группа $S L(p, R)$ имеет ранг $p-1$, т.е. имеет $p-1$ независимых операторов Казимира, матричный элемент $F$ теперь будет одновременно удовлетворять $p-1$ дифференциальным уравнениям на СЗ. Таким образом, этот

29) Здесь и далее мы опускаем тривиальный множитель, зависящий от космологической постоянной $\mu_{L} \mu_{R}$. 
матричный элемент решает квантовую задачу с гамильтонианами из пункта 6.1, отвечающими системе Тоды. Среди них, в частности, будет и квадратичный гамильтониан (6.9), приводящий к уравнению второго порядка - уравнению Шредингера, которое имеет вид

$$
\left(\frac{\partial^{2}}{\partial \phi^{2}}-2 \sum_{i} \mu_{i}^{L} \mu_{i}^{R} e^{\alpha_{i} \boldsymbol{\phi}}\right) \Psi^{(\boldsymbol{\lambda})}(\phi)=\lambda^{2} \Psi^{(\boldsymbol{\lambda})}(\phi)
$$

причем здесь функция $\Psi^{(\boldsymbol{\lambda})}(\boldsymbol{\phi})$ пропорциональна матричному элементу $e^{-\boldsymbol{\rho} \phi} F^{(\boldsymbol{\lambda})}(\boldsymbol{\phi})$, a $\mu_{i}^{R, L}$ - набор космологических постоянных. Явное вычисление матричного элемента дает решение этого уравнения в интегральной форме:

$$
\begin{aligned}
\Psi(\phi)= & e^{-\boldsymbol{\lambda} \boldsymbol{\phi}} \int \prod_{i<j} d x_{i j} \prod_{i=1}^{p-1} \Delta_{i}^{-\left(\boldsymbol{\lambda} \boldsymbol{\alpha}_{i}+1\right)}\left(x S^{-1}\right) \times \\
& \times \exp \left\{i \mu_{i}^{R} x_{i, i+1} e^{\boldsymbol{\alpha}_{i} \boldsymbol{\phi}}-i \mu_{p-i}^{L} \frac{\Delta_{i, i+1}\left(x S^{-1}\right)}{\Delta_{i}\left(x S^{-1}\right)}\right\} .
\end{aligned}
$$

Здесь $\boldsymbol{\alpha}_{i}$ - положительные простые корни, $S$ - внутренний автоморфизм группы $S L(p)$, отображаюший верхнетреугольные на нижнетреугольные матрицы $S_{i j} \equiv \delta_{i+j, p+1}$, $\Delta_{i}(A)$ - верхний главный минор матрицы $A$ размера $i \times i$, a $\Delta_{i, i+1}(A)$ - тот же самый минор, но матрицы, образованной из $A$ перестановкой $i$ и $i+1$ столбцов.

Теперь, исследуя асимптотики этой В $\Phi$, мы можем получить функции Хариш-Чандры [39]. Прежде всего заметим, что число различных асимптотик совпадает с числом элементов группы Вейля для $S L(p)$, хотя В $\Phi(6.42)$ и выражается только через простые корни. Таким образом, неудивительно, что функции Хариш-Чандры нумеруются элементами группы Вейля и связаны между собой действием этой группы. Вычисление асимптотик следует известной технике, развитой для случая разложения Ивасавы $[49,50]$, а результат имеет вид

$$
c_{s}(\boldsymbol{\lambda})=\prod_{\alpha \in \Delta^{+}} \frac{1}{\Gamma(1+s \boldsymbol{\lambda} \cdot \boldsymbol{\alpha})}
$$

где $s$ обозначает элемент группы Вейля, а произведение берется по всем положительным корням. Отношение этих функций Хариш-Чандры задает нам отражательную $S$-матрицу в теории.

Квантово-полевая система Лиувилля. В качестве следуюшего шага мы можем обобщить нашу конструкцию на случай аффинной алгебры. Действительно, рассматривая группу $\widehat{S L(2)}$ и “поточечное" разложение Гаусса (используемое при бозонизации аффинных алгебр, см. [52]), мы приходим к уравнению Шредингера для двумерной полевой системы Лиувилля. Это означает, что, вычисляя функции Хариш-Чандры в этом случае, мы найдем $S$-матрицу (или двухточечный коррелятор) двумерной теории поля Лиувилля. Действительно, выберем систему положительных корней следующим образом:

$$
\boldsymbol{\alpha}_{0}+n\left(\boldsymbol{\alpha}_{0}+\boldsymbol{\alpha}_{1}\right), \quad n\left(\boldsymbol{\alpha}_{0}+\boldsymbol{\alpha}_{1}\right), \quad \boldsymbol{\alpha}_{1}+n\left(\boldsymbol{\alpha}_{0}+\boldsymbol{\alpha}_{1}\right), \quad n=0,1,2, \ldots
$$


Обозначим

$$
\boldsymbol{\lambda} \cdot \boldsymbol{\alpha}_{0}=\frac{1}{2}-p+\tau, \quad \boldsymbol{\lambda} \cdot \boldsymbol{\alpha}_{1}=-\frac{1}{2}+p
$$

Теперь для функций Хариш-Чандры можно воспользоваться формулой (6.43), сдвинув только аргумент Г-функции на $1 / 2$, поскольку мы работаем с аффиннной алгеброй:

$$
c(\boldsymbol{\lambda})=\prod_{n \geq 0} \Gamma^{-1}(p+n \tau) \prod_{n \geq 1} \Gamma^{-1}(n \tau) \Gamma^{-1}(1-p+n \tau) .
$$

Это выражение, конечно, еще требует аккуратной регуляризации, но все бесконечные произведения равны нулю в соответствующей отражательной $S$-матрице (2-точечной функции):

$$
S(p)=\frac{c(-p)}{c(p)}=\frac{\Gamma(1+p) \Gamma\left(1+\frac{p}{\tau}\right)}{\Gamma(1-p) \Gamma\left(1-\frac{p}{\tau}\right)} .
$$

Это выражение совпадает с двухточечными функциями, полученными в работах [54, $55,53]$ совершенно иным способом ${ }^{30)}$.

Заметим, что если теперь ввести фундаментальньй строительный блок - функцию

$$
i(\boldsymbol{\lambda})=i(p, \tau) \sim \prod_{m, n \geq 0}(p+m+n \tau),
$$

где произведение идет только по одному (положительному) квадранту в плоскости $m, n$, то из него можно построить как функцию Хариш-Чандры (6.46), так и эллиптические тэта-фрункции

$$
\theta\left(p+\frac{1}{2}+\frac{\tau}{2}, \tau\right) \sim i(p, \tau) i(-p, \tau) i(p,-\tau) i(-p,-\tau)
$$

а также $q$-экспоненту

$$
e_{q}\left(e^{2 \pi i p}\right) \sim \frac{1}{i(p, \tau) i(-p,-\tau)}, \quad 1=e^{i \pi \tau},
$$

просто беря произведения по разным квадрантам. Все эти выражения, конечно, требуют подходящей регуляризации, поскольку неограниченные бесконечные произведения расходятся. Регуляризованные выражения можно найти в [105] и [53].

\section{Полубесконечные иерархии}

ПРИЛОЖКНИЕ 1

Мы воспроизведем здесь несколько технических вычислений, опушенных в пункте 2.3 (см. $[73,65])$. А именно, рассмотрим полубесконечную иерархию, заданную условием

$$
\tau_{n}=0, \quad n<0 .
$$

Это отвечает выбору элемента грассманниана в виде

$$
G=G_{0} P_{+}
$$

${ }^{30)}$ В обозначениях [53] $p=2 i P / b$ и $\tau=b^{2}$. 
где $P_{+}-$проектор на положительные состояния:

$$
P_{+}|n\rangle=\theta(n)|n\rangle
$$

который реализуется как фермионный оператор

$$
P_{+}=: \exp \left[\sum_{i<0} \psi_{i} \psi_{i}^{*}\right]:
$$

и обладает свойствами

$$
\begin{gathered}
P_{+} \psi_{-k}^{*}=\psi_{-k} P_{+}=0, \quad k>0 \\
{\left[P_{+}, \psi_{k}\right]=\left[P_{+}, \psi_{k}^{*}\right]=0, \quad k \geq 0} \\
P_{+}^{2}=P_{+} .
\end{gathered}
$$

Мы также выбираем $G_{0}$ в виде, включающем только положительные моды фермионов $\psi_{k}$ и $\psi_{k}^{*}$ с $k \geq 0$ :

$$
G_{0}=\exp \left\{\left(\int_{\gamma} A(z, w) \psi_{+}(z) \psi_{+}^{*}\left(w^{-1}\right) d z d w\right)-\sum_{i \geq 0} \psi_{i} \psi_{i}^{*}\right\}:
$$

где

$$
\psi_{+}(z)=\sum_{k \geq 0} \psi_{k} z^{k}, \quad \psi_{+}^{*}(z)=\sum_{k \geq 0} \psi_{k}^{*} z^{-k}
$$

и $\gamma$ - некоторая область интегрирования. Кроме того, нам понадобится проектор на отрицательные состояния

$$
P_{-}=\exp \left[-\sum_{i \geq 0} \psi_{i} \psi_{i}^{*}\right]:
$$

который обладает следующими свойствами:

$$
\begin{gathered}
P_{-} \psi_{k}=\psi_{k}^{*} P_{-}=0, \quad k \geq 0, \\
{\left[P_{-}, \psi_{-k}\right]=\left[P_{-}, \psi_{-k}^{*}\right]=0, \quad k>0,} \\
P_{-}^{2}=P_{-} .
\end{gathered}
$$

Вычислим состояние

$$
G_{0} P_{+} e^{\bar{H}(y)}|n\rangle \text {. }
$$

Как легко видеть, оно исчезает при $n<0$. Действительно, используя (2.8), (2.12) и (П1.6), можно получить, что при $n<0$ состояние

$$
e^{\bar{H}(\bar{t})}|n\rangle=\psi_{-n}^{*}(\bar{t}) \ldots \psi_{-1}^{*}(\bar{t}) e^{\bar{H}(\bar{t})}|0\rangle
$$

содержит только отрицательные моды $\psi_{-m}^{*}(m>0)$. Следовательно, действие $P_{+}$аннигилирует это состояние благодаря формуле (П1.5). Для $n \geq 0$, используя формулы (2.8), (2.12) и (П1.6), мы получаем

$$
P_{+} e^{\bar{H}(\bar{t})}|n\rangle=\psi_{n-1}(\bar{t}) \ldots \psi_{0}(\bar{t}) P_{+} e^{\bar{H}(\bar{t})}|0\rangle .
$$


Теперь мы используем тот факт, что

$$
P_{+} e^{\bar{H}(\bar{t})}|0\rangle=|0\rangle .
$$

Доказательство формулы (П1.15). Обозначим

$$
|\bar{t}\rangle=P_{+} e^{\bar{H}(\bar{t})}|0\rangle \text {. }
$$

Тогда согласно формулам (2.7) и (П1.5) имеем

$$
\frac{\partial}{\partial \bar{t}_{k}}|\bar{t}\rangle=P_{+} e^{\bar{H}(\bar{t})} \sum_{i=0}^{k-1} \psi_{i-k}^{*} \psi_{i}|0\rangle=0
$$

Поскольку $\left.|\bar{t}\rangle\right|_{\bar{t}_{k}=0}=|0\rangle$, формула (П1.15) доказана.

Таким образом, с использованием формул (П1.8), (П1.9) мы получаем

$$
\begin{aligned}
G_{0} P_{+} e^{\bar{H}(\bar{t})}|n\rangle= & G \psi(\bar{t}) \ldots \psi(\bar{t})|0\rangle= \\
= & \sum \frac{1}{m !} \int_{\gamma} \prod_{i=1}^{m} A\left(z_{i}, w_{i}\right) d z_{i} d w_{i} \psi_{+}\left(z_{1}\right) \ldots \psi_{+}\left(z_{m}\right) \times \\
& \times P_{-} \psi_{+}^{*}\left(w_{m}^{-1}\right) \ldots \psi_{+}^{*}\left(w_{1}^{-1}\right) \psi_{n-1}(\bar{t}) \ldots \psi_{0}(\bar{t})|0\rangle
\end{aligned}
$$

Мы видим теперь, что только член с $m=n$ дает ненулевой вклад в бесконечную сумму (П1.16). Действительно, при $m>n$ состояние $\psi_{+}^{*}\left(w_{m}^{-1}\right) \ldots \psi_{+}^{*}\left(w_{1}^{-1}\right) \psi_{n-1}(\bar{t}) \ldots$ $\ldots \psi_{0}(\bar{t})|0\rangle$ исчезает, поскольку в этом случае некоторые положительные моды в $\psi_{+}^{*}\left(w_{i}^{-1}\right)$ могут быть пододвинуты к вакууму $|0\rangle$ и поэтому обратят его в нуль. Обратно, при $m<n$ некоторые положительные моды в $\psi_{k}(-\bar{y})$ могут быть пододвинуты к проектору $P_{-}$и с помощью (П1.10) обратят его в нуль. Следовательно, получаем

$$
\begin{aligned}
G_{0} P_{+} e^{\bar{H}(\bar{t})}|n\rangle= & \frac{1}{n !} \int_{\gamma} \prod_{i=1}^{n} A\left(z_{i}, w_{i}\right) d z_{i} d w_{i} \psi_{+}\left(z_{1}\right) \ldots \psi_{+}\left(z_{n}\right) \times \\
& \times P_{-} \psi_{+}^{*}\left(w_{n}^{-1}\right) \ldots \psi_{+}^{*}\left(w_{1}^{-1}\right) \psi_{n-1}(\bar{t}) \ldots \psi_{0}(\bar{t})|0\rangle .
\end{aligned}
$$

Теперь мы используем следуюшее утверждение:

$$
\psi_{+}^{*}\left(w_{n}^{-1}\right) \ldots \psi_{+}^{*}\left(w_{1}^{-1}\right) \psi_{n-1}(\bar{t}) \ldots \psi_{0}(\bar{t})|0\rangle=\Delta(w) \exp \left[\sum_{j=1}^{n} \xi\left(\bar{t}, w_{j}\right)\right]|0\rangle .
$$

Доказательство формулы (П1.18). Поскольку число операторов рождения (по отношению к вакууму $|0\rangle) \psi_{i}(-\bar{y})$ равно числу операторов уничтожения $\psi_{+}^{*}\left(w_{j}^{-1}\right)$, ясно, что после нормального упорядочивания

$$
\psi_{+}^{*}\left(w_{n}^{-1}\right) \ldots \psi_{+}^{*}\left(w_{1}^{-1}\right) \psi_{n-1}(\bar{t}) \ldots \psi_{0}(\bar{t})|0\rangle=\mathrm{const} \cdot|0\rangle
$$

и

$$
\begin{aligned}
\text { const } & =\left\langle 0\left|\psi_{+}^{*}\left(w_{n}^{-1}\right) \ldots \psi_{+}^{*}\left(w_{1}^{-1}\right) \psi_{n-1}(\bar{t}) \ldots \psi_{0}(\bar{t})\right| 0\right\rangle= \\
& =\left.\operatorname{det}\left[\left\langle 0\left|\psi_{+}^{*}\left(w_{i}^{-1}\right) \psi_{j-1}(\bar{t})\right| 0\right\rangle\right]\right|_{i, j=1, \ldots, n}
\end{aligned}
$$


Теперь, используя формулы (2.13), (2.9), получаем

$$
\left\langle 0\left|\psi_{+}^{*}\left(w_{i}^{-1}\right) \psi_{j-1}(\bar{t})\right| 0\right\rangle=w_{i}^{j-1} e^{\xi\left(\bar{t}, w_{i}\right)} .
$$

Таким образом, окончательно имеем

$$
\text { const }=\operatorname{det}\left[w_{i}^{j-1} e^{\xi\left(\bar{t}, w_{i}\right)}\right]=\Delta(w) \exp \left[\sum_{j=1}^{n} \xi\left(\bar{t}, w_{j}\right)\right] .
$$

Теперь подставляя (П1.18) в (П1.17) и используя очевидный факт, что $P_{-}|0\rangle=|0\rangle$ и $\psi_{-}\left(z_{i}\right)|0\rangle=0$, мы получаем

$$
G_{0} P_{+} e^{\bar{H}(\bar{t})}|n\rangle=\frac{1}{n !} \int_{\gamma} \prod_{i=1}^{n} A\left(z_{i}, w_{i}\right) e^{\xi\left(\bar{t}, w_{i}\right)} d z_{i} d w_{i} \Delta(w) \psi\left(z_{1}\right) \ldots \psi\left(z_{n}\right)|0\rangle .
$$

Наконец, с помощью одной из основных формул работы [62] (которая может быть просто доказана в технике бозонизации)

$$
\psi\left(z_{1}\right) \ldots \psi\left(z_{n}\right)|0\rangle=\Delta(z) \exp \left[\bar{H}\left(\sum_{i=1}^{n} \epsilon\left(z_{i}\right)\right)\right]|0\rangle
$$

где $\epsilon\left(z_{i}\right)$ - вектор с компонентами $\epsilon_{k}\left(z_{i}\right)=(1 / k) z_{i}^{k}$, мы получаем желаемый результат:

$$
\begin{aligned}
G_{0} P_{+} e^{\bar{H}(\bar{t})}|n\rangle= & \frac{1}{n !} \int_{\gamma} \prod_{i=1}^{n} A\left(z_{i}, w_{i}\right) e^{\xi\left(\bar{t}, w_{i}\right)} d z_{i} d w_{i} \times \\
& \times \Delta(w) \Delta(z) \exp \left[\bar{H}\left(\sum_{i=1}^{n} \epsilon\left(z_{i}\right)\right)\right]|0\rangle .
\end{aligned}
$$

Итак, мы окончательно имеем

$$
\begin{aligned}
\tau_{n}(t, \bar{t}) & =\left\langle n\left|e^{H(x)} G_{0} P_{+} e^{\bar{H}(\bar{t})}\right| n\right\rangle= \\
& =\frac{1}{n !} \int_{\gamma} \Delta(w) \Delta(z) \prod_{i=1}^{n} A\left(z_{i}, w_{i}\right) e^{\xi\left(t, z_{i}\right)+\xi\left(\bar{t}, w_{i}\right)} d z_{i} d w_{i}
\end{aligned}
$$

Детерминантное представление. Используя формулы (2.8), (П1.7) и тот факт, что $\left[P_{+}, g_{0}\right]=0$, мы получаем

$$
\begin{aligned}
\tau_{n}(t, \bar{t}) & =\left\langle 0\left|\psi_{0}^{*} \ldots \psi_{n-1}^{*} e^{H(t)} G_{0} P_{+} e^{\bar{H}(\bar{t})} \psi_{n-1} \ldots \psi_{0}\right| 0\right\rangle= \\
& =\left\langle 0\left|e^{H(t)} \psi_{0}^{*}(-t) \ldots \psi_{n-1}^{*}(-t) P_{+} G_{0} P_{+} \psi_{n-1}(\bar{t}) \ldots \psi_{0}(\bar{t}) e^{\bar{H}(\bar{t})}\right| 0\right\rangle
\end{aligned}
$$

Поскольку $\psi_{i}^{*}(-t)$ и $\psi_{i}(\bar{t})$ содержат только положительные моды (см. формулы $(2.11)$ и (2.12)), из (П1.6) и (П1.15) можно получить

$$
\begin{aligned}
\tau_{n}(t, \bar{t}) & =\left\langle 0\left|\psi_{0}^{*}(-t) \ldots \psi_{n-1}^{*}(-t) G_{0} \psi_{n-1}(\bar{t}) \ldots \psi_{0}(\bar{t})\right| 0\right\rangle= \\
& =\left.\operatorname{det}\left[\left\langle 0\left|\psi_{i}^{*}(-t) G_{0} \psi_{j}(\bar{t})\right| 0\right\rangle\right]\right|_{i, j=0, \ldots, n-1}
\end{aligned}
$$


Те же самые аргументы, которые мы использовали для перехода от формулы (П1.16) к формуле (П1.17), примененные к уравнению (П1.23), приводят нас к заключению, что только линейный член в $A(z, w)$ дает вклад. Поэтому с помощью (П1.10) мы находим

$$
\begin{aligned}
\left\langle 0\left|\psi_{i}^{*}(-t) G \psi_{j}(\bar{t})\right| 0\right\rangle & =\int_{\gamma} A(z, w) d z d w\left\langle 0\left|\psi_{i}^{*}(-t) \psi_{+}(z) P_{-} \psi_{+}^{*}\left(w^{-1}\right) \psi_{j}(\bar{t})\right| 0\right\rangle= \\
& =\int_{\gamma} z^{i} w^{j} A(z, w) e^{\xi(t, z)+\xi(\bar{t}, w)} d z d w= \\
& =\partial_{t}^{i} \partial_{\bar{t}}^{j} \int_{\gamma} A(z, w) e^{\xi(t, z)+\xi(\bar{t}, w)} d z d w .
\end{aligned}
$$

Таким образом, окончательно выражение для $\tau$-функции в детерминантной форме имеет вид

$$
\tau_{n}(t, \bar{t})=\left.\operatorname{det}\left[\partial_{x_{1}}^{i} \partial_{\bar{t}_{1}}^{j} \int_{\gamma} A(z, w) e^{\xi(t, z)+\xi(\bar{t}, w)} d z d w\right]\right|_{i, j=0, \ldots, n-1} .
$$

\section{ПРИЛОЖЕНИЕ 2}

\section{Фундаментальные представления группы $S L_{q}(p)$}

Здесь мы обобщим описанную в пункте 5.1 структуру фундаментальных представлений на квантовый случай, т.е. группу $S L_{q}(p)$ с $q \neq 1$ (см. [35] и приведенные там ссылки).

Основное новое понятие, которое нам понадобится, - это определение $q$-антисимметризации как суммы по всем перестановкам:

$$
\left([1, \ldots, k]_{q}\right)=\sum_{P}(-q)^{\operatorname{deg} P}(P(1), \ldots, P(k)),
$$

где

$$
\operatorname{deg} P=\text { число инверсий в } P \text {. }
$$

Так, например, $q$-антисимметризация используется в определении $q$-детерминанта:

$$
\operatorname{det}_{q} A \sim A_{[1}^{[1} \ldots A_{p]_{q}}^{p]_{q}}=\sum_{P, P^{\prime}}(-q)^{\operatorname{deg} P+\operatorname{deg} P^{\prime}} \prod_{a} A_{P^{\prime}(a)}^{P(a)} .
$$

Заметим, что эта величина не обязательно совпадает с $A_{[1}^{1} \ldots A_{p]_{q}}^{p}$. Например, для $p=2$ формула (П2.3) дает

$$
\frac{1}{[2]}\left(A_{1}^{1} A_{2}^{2}-q A_{2}^{1} A_{1}^{2}-q A_{1}^{2} A_{2}^{1}+q^{2} A_{2}^{2} A_{1}^{1}\right)
$$

в то время как $q$-антисимметризация только по нижним индексам дала бы просто $A_{1}^{1} A_{2}^{2}-$ $q A_{2}^{1} A_{1}^{2}$. Более того, $A_{[1}^{1} A_{2]}^{1}=A_{1}^{1} A_{2}^{1}-q A_{2}^{1} A_{1}^{1}$ не обязано быть равным нулю, и даже $A_{[1}^{1} A_{1]_{q}}^{1}=(1-q)\left(A_{1}^{1}\right)^{2} \neq 0$.

"Нормальные" свойства $q$-антисимметризации восстанавливаются, если рассматривать в качестве $A_{j}^{i}$ элементы координатного кольца $A(G L(p))$ (алгебры функций на 
квантовой группе $\left.G L_{q}(p)\right)$. Такие матричные элементы удовлетворяют следующим коммутационным соотношениям (в частном случае $p=2$ сводящимся к (4.39)) [26]:

$$
\begin{gathered}
\forall i, \forall j_{1}<j_{2} \quad A_{\left[j_{1}\right.}^{i} A_{\left.j_{2}\right]_{q}}^{i}=0 \quad(a b=q b a, \quad c d=q d c), \\
\forall i_{1}<i_{2}, \forall j \quad A_{j}^{\left[i_{1}\right.} A_{j}^{\left.i_{2}\right]_{q}}=0 \quad(a c=q c a, \quad b d=q d b), \\
\forall i_{1} \neq i_{2}, j_{1} \neq j_{2} \quad A_{j_{2}}^{i_{1}} A_{j_{1}}^{i_{2}}=A_{j_{1}}^{i_{2}} A_{j_{2}}^{i_{1}} \quad(b c=c b), \\
\forall i_{1}<i_{2}, j_{1}<j_{2} \\
A_{j_{1}}^{i_{1}} A_{j_{2}}^{i_{2}}-A_{j_{2}}^{i_{2}} A_{j_{1}}^{i_{1}}=\left(q-q^{-1}\right) A_{j_{2}}^{i_{1}} A_{j_{1}}^{i_{2}} \quad\left(a d-d a=\left(q-q^{-1}\right) b c\right) .
\end{gathered}
$$

Для $A \in G L_{q}(p)$

$$
\operatorname{det}_{q} A=A_{[1}^{1} \ldots A_{p]_{q}}^{p}=A_{1}^{[1} \ldots A_{p}^{p]_{q}}
$$

и $A_{[1}^{i_{1}} \ldots A_{k]_{q}}^{i_{k}}=0$, если любые два верхних индекса совпадают (и только при условии, что все нижние различны: даже для $A \in G L_{q}(p)$ все еше верно, что $A_{[1}^{1} A_{1]_{q}}^{1}=$ $\left.(1-q)\left(A_{1}^{1}\right)^{2} \neq 0\right)$.

Понятие $q$-антисимметризации важно для построения фундаментальных представлений, поскольку $k$-е фундаментальное представление $F^{(k)}$ для $S L_{q}(p)$ является $q$-антисимметричной степенью $F=F^{(1)}$ : при $q \neq 1$ вместо (5.2) мы имеем

$$
F^{(k)}=\left\{\Psi_{i_{1} \ldots i_{k}}^{(k)} \sim \psi_{\left[i_{1}\right.} \ldots \psi_{\left.i_{k}\right]_{q}}, \quad i_{1}<\cdots<i_{k}\right\} .
$$

Заметим, что теперь необходимо явно потребовать, чтобы все $i_{a}$ были различны.

Все формулы типа (5.4) для сплетаюших операторов в квантовом случае остаются неизменными, если заменить в них антисимметризацию $q$-антисимметризацией (и очевидным образом определить $q$ - - -символ). Вместо (5.7) мы теперь получаем

$$
g^{(k)}\left(\begin{array}{c}
i_{1} \ldots i_{k} \\
j_{1} \ldots j_{k}
\end{array}\right) \sim \operatorname{det}_{q} g_{j_{b}}^{i_{a}}, \quad i_{1}<\cdots<i_{k} \text { или } j_{1}<\cdots<j_{k},
$$

а (5.8) преврашается в

$$
\begin{gathered}
g^{(k)}\left(\begin{array}{c}
i_{1} \ldots i_{k} \\
{\left[j_{1} \ldots j_{k}\right.}
\end{array}\right) g^{\left(k^{\prime}\right)}\left(\begin{array}{c}
i_{1}^{\prime} \ldots i_{k^{\prime}}^{\prime} \\
\left.j_{k+1}\right]_{q} j_{1}^{\prime} \ldots j_{k^{\prime}-1}^{\prime}
\end{array}\right)= \\
=g^{(k+1)}\left(\begin{array}{c}
i_{1} \ldots i_{k}\left[i_{k}^{\prime}\right. \\
j_{1} \ldots j_{k+1}
\end{array}\right) g^{\left(k^{\prime}-1\right)}\left(\begin{array}{c}
\left.i_{1}^{\prime} \ldots i_{k^{\prime}-1}^{\prime}\right]_{q} \\
j_{1}^{\prime} \ldots j_{k^{\prime}-1}^{\prime}
\end{array}\right), \\
i_{1}<\cdots<i_{k} \text { или } j_{1}<\cdots<j_{k}, \\
i_{1}^{\prime}<\cdots<i_{k^{\prime}}^{\prime} \text { или } j_{k+1}<j_{1}^{\prime}<\cdots<j_{k^{\prime}-1}^{\prime}, \\
i_{1}<\cdots<i_{k}<i_{k^{\prime}}^{\prime} \text { или } j_{1}<\cdots<j_{k+1}, \\
i_{1}^{\prime}<\cdots<i_{k^{\prime}-1}^{\prime} \text { или } j_{1}^{\prime}<\cdots<j_{k^{\prime}-1}^{\prime} .
\end{gathered}
$$


В точности, как и $(5.8)$, это просто тождество на матрицы из $G L_{q}(p)^{31)}$. Новая по сравнению с (5.8) черта - явное ограничение индексов $i, j, i^{\prime}, j^{\prime}$, которое делает переход на язык производящих функций более сложным.

Заметим, что, подобно классическому случаю, из квантовых миноров (П2.7) можно построить локальные координаты на квантовом пространстве флагов [106]. Как и в классическом случае, эти координаты удовлетворяют набору (квантовых) билинейных тождеств (Плюккера) и являются квантовым обобщением плюккеровых координат [107]. К сожалению, до сих пор неизвестен согласованный способ параметризации плюккеровых координат временами.

Теперь мы хотели бы подробнее обсудить формулу (П2.6). Мы начнем с классического случая, поскольку структура представлений практически не меняется при квантовании.

Алгебра Ли $S L(p)$ генерируется операторами $T_{ \pm \alpha}$ и картановскими операторами $H_{\boldsymbol{\beta}}$, так что $\left[H_{\beta}, T_{ \pm \alpha}\right]= \pm(1 / 2)(\boldsymbol{\alpha} \boldsymbol{\beta}) T_{ \pm \alpha}$. Все элементы представлений являются собственными функциями картановских генераторов $H_{\boldsymbol{\beta}}, H_{\boldsymbol{\beta}}|\boldsymbol{\lambda}\rangle=(1 / 2)(\boldsymbol{\beta} \boldsymbol{\lambda})|\boldsymbol{\lambda}\rangle$. Старшие векторы $\boldsymbol{\mu}_{k}$ представлений $F^{(k)}$ образуют систему, "дуальную" к nростым.м корням $\boldsymbol{\alpha}_{i}$, $i=1, \ldots, r:\left(\boldsymbol{\mu}_{i} \boldsymbol{\alpha}_{j}\right)=\delta_{i j}$, причем

$$
\rho \equiv \frac{1}{2} \sum_{\alpha>0} \alpha=\sum_{i} \mu_{i}
$$

Представление $F^{(1)}$ содержит состояния вида

$$
\psi_{i}=T_{-(i-1)} \ldots T_{-2} T_{-1} \psi_{1}, \quad i=1, \ldots, p
$$

Более того,

$$
T_{-i} \psi_{j}=\delta_{i j} \psi_{i+1}
$$

(таким образом, для $T_{-}=\sum_{i=1}^{r} T_{-i}$ имеем $T_{-}^{i} \psi_{j}=\psi_{j+i}$ и получается уравнение (5.1)) и

$$
\boldsymbol{\lambda}\left(\psi_{i}\right)=\boldsymbol{\mu}_{1}-\boldsymbol{\alpha}_{1}-\cdots-\boldsymbol{\alpha}_{i_{1}},
$$

здесь $T_{ \pm i} \equiv T_{ \pm \alpha_{i}}$ - генераторы, ассоциированные с простыми корнями. Обозначим соответствующий базис в алгебре Картана через $H_{i}=H_{\alpha_{i}}, H_{i}|\boldsymbol{\lambda}\rangle=(1 / 2)\left(\boldsymbol{\alpha}{ }_{i} \boldsymbol{\lambda}\right)|\boldsymbol{\lambda}\rangle=$ $\lambda_{i}|\boldsymbol{\lambda}\rangle$, тогда

$$
\lambda_{i}^{(j)} \equiv \lambda_{i}\left(\psi_{j}\right)=\frac{1}{2}\left(\delta_{i j}-\delta_{i, j-1}\right)
$$

31) Здесь важно, что $g$ принадлежит некоторой $G L_{q}(p)$, т.е. ее элементы имеют подходящие коммутационные соотношения. Это, конечно, подразумевалось при выводе (П2.8), где предполагалось, что $g$ является групповым элементом. Не будь этого, формула (П2.7) должна была бы пониматься в духе (П2.3) (в частности, нужно было бы писать " $u$ " вместо “ $и л u$ " в (П2.7)), и мы пришли бы к противоречию с (П2.8). Чтобы прояснить это место, рассмотрим пример $k=k^{\prime}=1$, так что (П2.8) превращается в

$$
g_{\left[j_{1}\right.}^{i} g_{\left.j_{2}\right]_{q}}^{i^{\prime}}=g^{(2)}\left(\begin{array}{c}
i i^{\prime} \\
j_{1} j_{2}
\end{array}\right)
$$

и левая часть этой формулы равна $g_{j_{1}}^{i} g_{j_{2}}^{i^{\prime}}-q g_{j_{2}}^{i} g_{j_{1}}^{i^{\prime}}$, в то время как правая должна интерпретироваться как $g_{j_{1}}^{i} g_{j_{2}}^{i^{\prime}}-q g_{j_{2}}^{i} g_{j_{1}}^{i^{\prime}}-q g_{j_{1}}^{i^{\prime}} g_{j_{2}}^{i}+q^{2} g_{j_{2}}^{i^{\prime}} g_{j_{1}}^{i}$. 
Эта формула вместе с (П2.11) и коммутационными соотношениями алгебры дает $\left\|\psi_{i}\right\|^{2}=1$. Поскольку закон коумножения в классическом случае - это просто $\Delta(T)=T \otimes I+I \otimes T$, то $\psi_{[1} \ldots \psi_{k]}$ - все векторы старшего веса (т.е. они обрашаются в нуль всеми $\Delta_{k}\left(T_{+i}\right)$ и, таким образом, всеми $\left.\Delta_{k}\left(T_{+\alpha}\right)\right)$.

Квантовая УОА $U_{q}(S L(p))$ задается генераторами Шевалле $T_{ \pm i}$, отвечающими простым корням, а также $q^{ \pm H_{i}}$ с определяющими коммутационными соотношениями $\left(a_{i j}\right.$ - матрица Картана $\left.S L(p)\right)[108,109,98]$

$$
\begin{gathered}
q^{H_{i}} T_{ \pm j} q^{-H_{i}}=q^{ \pm a_{i j}} T_{ \pm j}, \\
{\left[T_{+i}, T_{-j}\right]=\delta_{i j} \frac{q^{2 H_{i}}-q^{-2 H_{i}}}{q-q^{-1}}}
\end{gathered}
$$

и законом коумножения

$$
\begin{gathered}
\Delta\left(T_{ \pm i}\right)=q^{H_{i}} \otimes T_{ \pm i}+T_{ \pm i} \otimes q^{-H_{i}} \\
\Delta\left(q^{ \pm H_{i}}\right)=q^{ \pm H_{i}} \otimes q^{ \pm H_{i}} .
\end{gathered}
$$

Генераторы Шевалле, кроме того, подчиняются дополнительным соотношениям Серpa $[108,109]$. Если перейти от базиса Шевалле к базису генераторов, отвечаюших всем корням, то тождества Серра не нужны (они преврашаются в соотношения алгебры). В этом случае, однако, закон коумножения для $T_{ \pm \alpha}$, который легко считывается с (П2.16), оказывается довольно сложным. К примеру, для генератора, отвечаюшего корню “веса $2 " \boldsymbol{\alpha}$, т.е. $T_{ \pm \alpha}= \pm\left[T_{ \pm \alpha_{i}}, T_{ \pm \alpha_{i+1}}\right]$, мы получаем

$$
\begin{aligned}
\Delta\left(T_{-\alpha}\right)=- & {\left[\Delta\left(T_{\alpha_{i}}\right), \Delta\left(T_{\alpha_{i+1}}\right)\right]=q^{H_{\alpha}} \otimes T_{\alpha}+T_{\alpha} \otimes q^{-H_{\alpha}}+\left(q^{1 / 2}-q^{-1 / 2}\right) \times } \\
& \times\left[\left(T_{-\alpha_{i}} \otimes T_{-\alpha_{i+1}}\right)\left(q^{H_{i+1}} \otimes q^{-H_{i}}\right)-\right. \\
& \left.-\left(T_{-\alpha_{i+1}} \otimes T_{-\alpha_{i}}\right)\left(q^{H_{i}} \otimes q^{-H_{i+1}}\right)\right] .
\end{aligned}
$$

При заданном правиле коумножения можно легко проверить справедливость формулы (П2.6). Например, для $F^{(2)}$ имеем

$$
\Delta\left(T_{+i}\right)\left(\psi_{1} \psi_{2}-q \psi_{2} \psi_{1}\right)=\delta_{i, 1}\left(q^{\lambda_{1}^{(1)}} \psi_{1} \psi_{1}-q^{1-\lambda_{1}^{(1)}} \psi_{1} \psi_{1}\right)=0
$$

поскольку $\lambda_{1}^{(1)}=1 / 2$. Таким образом, $\Psi_{12}^{(2)} \equiv \psi_{[1} \psi_{2]_{q}}$, действительно, является вектором старшего веса. Подобным образом $(i<j)$ находим

$$
\begin{aligned}
\Delta\left(T_{-l}\right) \Psi_{i j}^{(2)}= & \Delta\left(T_{-l}\right)\left(\psi_{i} \psi_{j}-q \psi_{j} \psi_{i}\right)= \\
= & \delta_{l i} q^{-\lambda_{i}^{(j)}}\left(\psi_{i+1} \psi_{j}-q^{1+2 \lambda_{i}^{(j)}} \psi_{j} \psi_{i+1}\right)+ \\
& +\delta_{l j} q^{\lambda_{j}^{(i)}}\left(\psi_{i} \psi_{j+1}-q^{1-2 \lambda_{j}^{(i)}} \psi_{j+1} \psi_{i}\right) .
\end{aligned}
$$

Согласно (П2.14) для $i<j$ всегда $\lambda_{j}^{(i)}=0$, в то время как $\lambda_{i}^{(j)} \neq 0$, только если $j=i+1$, причем $\lambda_{i}^{(i+1)}=-1 / 2$. Таким образом, имеем

$$
\Delta\left(T_{-l}\right) \Psi_{i j}^{(2)}=\delta_{l j} \Psi_{i, j+1}^{(2)}+\delta_{l i} \Psi_{i+1, j}^{(2)}\left(1-\delta_{i+1, j}\right) .
$$


Отсюда следуют правила для действия всех $\Delta\left(T_{-\alpha}\right)$. Нетрудно явно описать действие всех $\Delta\left(T_{+i}\right)$ и проделать то же самое также и для других представлений $F^{(k)}$.

\section{ПРИЛОЖЕНИЕ 3}

\section{Явный пример: $\tau_{2}$ для $S L_{q}(p)$}

Рассмотрим простейшую $\tau$-функцию для группы $S L_{q}(p)$ в простейшем нетривиальном втором фундаментальном представлении [38]. Обозначим через $\{u\}$ и $\{v\}$ поднаборы $\{s\}$ такие, что $i(s)=1$ и $i(s)=2$, соответственно. Тогда имеем

$$
\begin{aligned}
\tau_{2}= & \tau_{1}^{00}\left(\left\{q \xi_{u}\right\},\left\{q^{-1} \xi_{v}\right\}, \xi_{s} ;\left\{\bar{\xi}_{u}\right\},\left\{\bar{\xi}_{v}\right\}, \bar{\xi}_{s}\right) \tau_{1}^{11}\left(\left\{\xi_{u}\right\},\left\{\xi_{v}\right\}, \xi_{s} ;\left\{q \bar{\xi}_{u}\right\},\left\{\bar{\xi}_{v}\right\}, \bar{\xi}_{s}\right)- \\
& -q \tau_{1}^{01}\left(\left\{q \xi_{u}\right\},\left\{q^{-1} \xi_{v}\right\}, \xi_{s} ;\left\{\bar{\xi}_{u}\right\},\left\{\bar{\xi}_{v}\right\}, \bar{\xi}_{s}\right) \tau_{1}^{10}\left(\left\{\xi_{u}\right\},\left\{\xi_{v}\right\}, \xi_{s} ;\left\{q^{-1} \bar{\xi}_{u}\right\},\left\{q \bar{\xi}_{v}\right\}, \bar{\xi}_{s}\right)- \\
& -q \tau_{1}^{10}\left(\left\{q^{-1} \xi_{u}\right\},\left\{\xi_{v}\right\}, \xi_{s} ;\left\{\bar{\xi}_{u}\right\},\left\{\bar{\xi}_{v}\right\}, \bar{\xi}_{s}\right) \tau_{1}^{01}\left(\left\{\xi_{u}\right\},\left\{\xi_{v}\right\}, \xi_{s} ;\left\{q \bar{\xi}_{u}\right\},\left\{\bar{\xi}_{v}\right\}, \bar{\xi}_{s}\right)+ \\
& +q^{2} \tau_{1}^{11}\left(\left\{q^{-1} \xi_{u}\right\},\left\{\xi_{v}\right\}, \xi_{s} ;\left\{\bar{\xi}_{u}\right\},\left\{\bar{\xi}_{v}\right\}, \bar{\xi}_{s}\right) \tau_{1}^{00}\left(\left\{\xi_{u}\right\},\left\{\xi_{v}\right\}, \xi_{s} ;\left\{q^{-1} \bar{\xi}_{u}\right\},\left\{q \bar{\xi}_{v}\right\}, \bar{\xi}_{s}\right)
\end{aligned}
$$

Здесь $\xi_{s}$ обозначает все времена с $i(s)>2$. Рассмотрим теперь параметризацию А. В формуле (П3.1) каждый набор $\{u\}$ и $\{v\}$ состоит из единственного элемента: $\{u\}=$ $\{s=1\},\{v\}=\{s=2\}$. Получаем

$$
\begin{aligned}
\tau_{2}= & \tau_{1}^{00}\left(q \xi_{1}, q^{-1} \xi_{2}, \xi_{i} ; \bar{\xi}_{1}, \bar{\xi}_{2}, \bar{\xi}_{i}\right) \tau_{1}^{11}\left(\xi_{1}, \xi_{2}, \xi_{i} ; q \bar{\xi}_{1}, \bar{\xi}_{2}, \bar{\xi}_{i}\right)- \\
& -q \tau_{1}^{01}\left(q \xi_{1}, q^{-1} \xi_{2}, \xi_{i} ; \bar{\xi}_{1}, \bar{\xi}_{2}, \bar{\xi}_{i}\right) \tau_{1}^{10}\left(\xi_{1}, \xi_{2}, \xi_{i} ; q^{-1} \bar{\xi}_{1}, q \bar{\xi}_{2}, \bar{\xi}_{i}\right)- \\
& -q \tau_{1}^{10}\left(q^{-1} \xi_{1}, \xi_{2}, \xi_{i} ; \bar{\xi}_{1}, \bar{\xi}_{2}, \bar{\xi}_{i}\right) \tau_{1}^{01}\left(\xi_{1}, \xi_{2}, \xi_{i} ; q \bar{\xi}_{1}, \bar{\xi}_{2}, \bar{\xi}_{i}\right)+ \\
& +q^{2} \tau_{1}^{11}\left(q^{-1} \xi_{1}, \xi_{2}, \xi_{i} ; \bar{\xi}_{1}, \bar{\xi}_{2}, \bar{\xi}_{i}\right) \tau_{1}^{00}\left(\xi_{1}, \xi_{2}, \xi_{i} ; q^{-1} \bar{\xi}_{1}, q \bar{\xi}_{2}, \bar{\xi}_{i}\right)
\end{aligned}
$$

С помошью операторов

$\mathcal{D}_{1}^{L} \equiv M_{1}^{-} D_{1} \otimes I, \quad \mathcal{D}_{1}^{R} \equiv M_{1}^{+} M_{2}^{-} \otimes D_{1}, \quad \overline{\mathcal{D}}_{1}^{L} \equiv \bar{D}_{1} \otimes \bar{M}_{1}^{-} \bar{M}_{2}^{+}, \quad \overline{\mathcal{D}}_{1}^{R} \equiv I \otimes \bar{M}_{1}^{+} \bar{D}_{1}$

выражение (П3.2) может быть переписано в более компактной форме

$$
\begin{aligned}
\tau_{2} & =\left(\mathcal{D}_{1}^{R} \overline{\mathcal{D}}_{1}^{R}-q \mathcal{D}_{1}^{L} \overline{\mathcal{D}}_{1}^{R}-q \mathcal{D}_{1}^{R} \overline{\mathcal{D}}_{1}^{L}+q^{2} \mathcal{D}_{1}^{L} \overline{\mathcal{D}}_{1}^{L}\right) \tau_{1} \otimes \tau_{1}= \\
& =\left(\mathcal{D}_{1}^{R}-q \mathcal{D}_{1}^{L}\right) \cdot\left(\overline{\mathcal{D}}_{1}^{R}-q \overline{\mathcal{D}}_{1}^{L}\right) \tau_{1} \otimes \tau_{1} .
\end{aligned}
$$

Эти операторы удовлетворяют коммутационным соотношениям, образуюшим алгебру, подобную $\theta$ и $\chi$ в (4.56): $\mathcal{D}_{1}^{L} \mathcal{D}_{1}^{R}=q \mathcal{D}_{1}^{R} \mathcal{D}_{1}^{L}, \overline{\mathcal{D}}_{1}^{L} \overline{\mathcal{D}}_{1}^{R}=q \overline{\mathcal{D}}_{1}^{R} \overline{\mathcal{D}}_{1}^{L}$. Формула для $\tau_{2}$ может быть переписана в более "инвариантном" виде в терминах операторов:

$$
\begin{aligned}
& \mathbb{D}_{i}^{L} \equiv D_{i} \otimes I, \quad \mathbb{D}_{i}^{R} \equiv \prod_{j} M_{j}^{-\vec{\alpha}_{i} \vec{\alpha}_{j}} \otimes D_{i}, \\
& \overline{\mathbb{D}}_{i}^{L} \equiv \bar{D}_{i} \otimes \prod_{j} \bar{M}_{j}^{-\vec{\alpha}_{i} \vec{\alpha}_{j}}, \quad \overline{\mathbb{D}}_{i}^{R} \equiv I \otimes \bar{D}_{i},
\end{aligned}
$$

коммутируюших как

$$
\mathbb{D}_{i}^{L} \mathbb{D}_{j}^{R}=q^{\vec{\alpha}_{i} \vec{\alpha}_{j}} \mathbb{D}_{j}^{R} \mathbb{D}_{i}^{L}, \quad \overline{\mathbb{D}}_{i}^{L} \overline{\mathbb{D}}_{j}^{R}=q^{\vec{\alpha}_{i} \vec{\alpha}_{j}} \overline{\mathbb{D}}_{j}^{R} \overline{\mathbb{D}}_{i}^{L} .
$$


Действительно, тогда получаем

$$
\tau_{2}=M_{1}^{-} \otimes \bar{M}_{1}^{+}\left(\mathbb{D}_{1}^{R}-q \mathbb{D}_{1}^{L}\right) \cdot\left(\overline{\mathbb{D}}_{1}^{R}-q \overline{\mathbb{D}}_{1}^{L}\right) \tau_{1} \otimes \tau_{1}
$$

\section{Построение функции Уиттекера для группы $S L(p)$}

ПРИЛОЖЕНИЕ 4

Построим В $\Phi$ системы Тоды, отвечаюшей групте $S L(p)$ [39]. Начнем с обозначений и определений. Стандартными источниками для нас являются учебники $[110,111]$.

\section{П. 4.1. Обозначения}

Алгебра Ли группы Ли $S L(p)$ полностью задается следуюшими коммутационными соотношениями генераторов, отвечаюших простым корням (генераторов Шевалле):

$$
\left[T_{ \pm i}, T_{0, j}\right]=\mp A_{i j} T_{ \pm, i}, \quad\left[T_{+i}, T_{-j}\right]=\delta_{i j} T_{0, j}, \quad i, j=1, \ldots, p-1,
$$

а также соотношениями Серра на них:

$$
\operatorname{ad}_{T_{ \pm i}}^{1-A_{i j}}\left(T_{ \pm j}\right)=0
$$

где

$$
\operatorname{ad}_{x}^{k} \equiv \underbrace{[x,[x, \ldots,[x, y] \ldots]]}_{k \text { раз }} .
$$

Остальные коммутационные соотношения могут быть получены из (П4.1), (П4.2), причем генераторы, отвечающие положительным (отрицательным) непростым корням, могут быть построены из генераторов Шевалле, отвечающих положительным (отрицательным) корням, при помоши явной формулы $\left[T_{\boldsymbol{\alpha}}, T_{\boldsymbol{\beta}}\right]=N_{\boldsymbol{\alpha}, \boldsymbol{\beta}} T_{\boldsymbol{\alpha}+\boldsymbol{\beta}}$. Генератор $T_{\boldsymbol{\alpha}+\boldsymbol{\beta}}$ здесь отвечает непростому корню $\boldsymbol{\alpha}+\boldsymbol{\beta}$, а $N_{\boldsymbol{\alpha}, \boldsymbol{\beta}}$ - некоторые ненулевые структурные константы. При использовании генераторов, отвечающих непростым корням, тождества Серра заменяются подходящими соотношениями алгебры Ли.

Мы будем использовать также следуюшие обозначения: $\boldsymbol{\alpha}_{i}-$ простые корни в соответствуюшей алгебре Ли, $A_{i j} \equiv \boldsymbol{\alpha}_{i} \cdot \boldsymbol{\alpha}_{j}$-матрица Картана, $\boldsymbol{\mu}_{i}$ - фундаментальные веса, которые по определению лежат в дуальной решетке $\boldsymbol{\mu}_{i} \cdot \alpha_{j}=\delta_{i j}\left(\right.$ т.е. $\boldsymbol{\mu}_{i}=A_{i j}^{-1} \boldsymbol{\alpha}_{j}$ ), а

$$
\rho \equiv \frac{1}{2} \sum_{\boldsymbol{\alpha} \in \Delta^{+}} \boldsymbol{\alpha}=\sum_{i} \boldsymbol{\mu}_{i}
$$

где $\Delta^{+}-$набор всех положительных корней. В этих обозначениях $\phi_{i}=-\boldsymbol{\mu}_{i} \cdot \boldsymbol{\phi}$. Kорни $\boldsymbol{\alpha}_{i}$ могут быть также рассмотрены как векторы в $p$-мерной картановской плоскости группы $G L(p): \boldsymbol{\alpha}_{i}=\mathbf{e}_{i+1}-\mathbf{e}_{i}, \mathbf{e}_{i} \cdot \mathbf{e}_{j}=\delta_{i j}$.

Для определения операторов Казимира фиксируем некоторое представление $\rho$ УОА $U(S L(p))$. Определим $L$-оператор формулой [26]

$$
L \equiv \sum_{\boldsymbol{\alpha} \in \Delta} \rho\left(T_{\boldsymbol{\alpha}}\right) \otimes T_{-\boldsymbol{\alpha}}+\sum_{i} A_{i j}^{-1} \rho\left(T_{i}\right) \otimes T_{j} .
$$

3 Теоретическая и математическая физика, т. 114, № 2, 1998 г. 
Тогда $k$-й оператор Казимира может быть задан выражением

$$
C_{k} \equiv \operatorname{tr}_{\rho} L^{k}
$$

где след берется по представлению $\rho$. В действительности, поскольку результат не зависит от представления $\rho$, можно выбрать простейшее первое фундаментальное представление. Тогда операторы Казимира вычисляются весьма просто. В частности, квадратичньй оператор Казимира имеет вид

$$
C_{2}=\sum_{\boldsymbol{\alpha} \in \Delta} T_{\boldsymbol{\alpha}} T_{-\boldsymbol{\alpha}}+\sum_{i j}^{p-1} A_{i j}^{-1} T_{0, i} T_{0, j}
$$

где первая сумма идет по всем (положительным и отрицательным) корням.

\section{П 4.2. Представления}

Для произвольной группы $S L(p)$ можно определить (правое) регулярное представление только в общих терминах группы, действующей на пространстве алгебры функций:

$$
\pi_{\text {reg }}(h) f(g)=f(g h) .
$$

Поэтому мы будем в основном использовать групповые (не алгебраические) термины (см., однако, [39]). Оказывается, что для общей группы $S L(p)$ возможно описывать ограничение пространства функций на неприводимые представления. Для этого надо рассмотреть представления, индуцированные одномерными представлениями борелевской подгруппы. Это ограничивает пространство всех функций на функции, удовлетворяющие свойству ковариантности:

$$
f_{\lambda}(b g)=\chi_{\lambda}(b) f_{\lambda}(g)
$$

где $b$ - элемент борелевской подгруппы нижнетреугольных матриц, а $\chi_{\lambda}$ - характер борелевской подгруппы вида

$$
\chi_{\lambda}(b)=\prod_{i=1}^{p-1}\left|b_{i i}\right|^{(\boldsymbol{\lambda}-\boldsymbol{\rho}) \mathbf{e}_{i}}\left(\operatorname{sign} b_{i i}\right)^{\epsilon_{i}}
$$

где $\epsilon_{i}$ равны 0 или 1 . Для простоты мы рассмотрим только представления без знаковых факторов, хотя обший случай также легко исследуется. Описанные представления принадлежат главной сферической серии.

Таким образом, представления даются ограничением пространства функций на функции, определенные на фактор-группе $B \backslash G$, которая, может быть идентифицирована с (нильпотентной) подгруппой строго верхнетреугольных матриц $N_{+}$. При данном $\lambda$ существует естественная эрмитова билинейная форма на пространстве матричных элементов $X$, которая задана просто плоской мерой:

$$
\left\langle f_{L} \mid f_{R}\right\rangle_{\lambda}=\int_{X=B \backslash G} \overline{f_{L, \lambda}(x)} f_{R, \lambda}(x) \prod_{i j} d x_{i j}
$$


Эта форма становится скалярным произведением при чисто мнимых $\lambda$, что дает унитарные неприводимые представления основной серии.

\section{П 4.3. Гамильтониан и уравнение Шредингера}

Рассмотрим следуюшие условия редукции, аналогичные (6.30):

$$
T_{+, i}\left|\psi_{R}\right\rangle=i \mu_{i}^{R}\left|\psi_{R}\right\rangle
$$

и

$$
\left\langle\psi_{L}\right| T_{-, i}=i \mu_{i}^{L}\left\langle\psi_{L}\right|,
$$

где $\mu_{i}^{R, L}$ - космологические постоянные. Эти условия задаются только генераторами Шевалле, поскольку все остальные легко получаются из коммутационных соотношений алгебры. Точнее, действие всех генераторов, отвечающих непростым корням, просто дает нуль. Теперь нетрудно получить, что

$$
\begin{aligned}
& \left(\boldsymbol{\lambda}^{2}-\boldsymbol{\rho}^{2}\right) F^{(\boldsymbol{\lambda})}(\boldsymbol{\phi}) \equiv\left(\boldsymbol{\lambda}^{2}-\boldsymbol{\rho}^{2}\right)\left\langle\psi_{L}\left|e^{-\boldsymbol{\mu}_{i} \boldsymbol{\phi} T_{0, i}}\right| \psi_{R}\right\rangle=\left\langle\psi_{L}\left|e^{-\boldsymbol{\mu}_{i} \boldsymbol{\phi} T_{0, i}} C_{2}\right| \psi_{R}\right\rangle= \\
& \quad=\left\langle\psi_{L}\left|e^{-\boldsymbol{\mu}_{i} \boldsymbol{\phi} T_{0, i}}\left(2 \sum_{\boldsymbol{\alpha} \in \Delta^{+}} T_{\boldsymbol{\alpha}} T_{-\boldsymbol{\alpha}}+2 \sum_{i j} A_{i j}^{-1} T_{0, j}+\sum_{i j} A_{i j}^{-1} T_{0, i} T_{0, j}\right)\right| \psi_{R}\right\rangle= \\
& =\left(\frac{\partial^{2}}{\partial \boldsymbol{\phi}^{2}}+2 \sum_{i} \partial\left(\boldsymbol{\alpha}_{i} \boldsymbol{\phi}\right)-2 \sum_{i} \mu_{i}^{L} \mu_{i}^{R} e^{\boldsymbol{\alpha}_{i} \boldsymbol{\phi}}\right)\left\langle\psi_{L}\left|e^{-\boldsymbol{\mu}_{i} \boldsymbol{\phi} T_{0, i}}\right| \psi_{R}\right\rangle
\end{aligned}
$$

и $\Psi^{(\boldsymbol{\lambda})}(\phi)=e^{-\boldsymbol{\rho} \phi} F^{(\boldsymbol{\lambda})}(\phi)$ удовлетворяет уравнению Шредингера для цепочки Тоды

$$
\left(\frac{\partial^{2}}{\partial \phi^{2}}-2 \sum_{i} \mu_{i}^{L} \mu_{i}^{R} e^{\boldsymbol{\alpha}_{i} \boldsymbol{\phi}}\right) \Psi^{(\boldsymbol{\lambda})}(\boldsymbol{\phi})=\lambda^{2} \Psi^{(\boldsymbol{\lambda})}(\boldsymbol{\phi}) .
$$

\section{П4.4. Решение уравнения Шредингера}

Для того чтобы явно вычислить функцию Уиттекера, мы должны явно построить решение условий (П4.10) и (П4.11). Здесь мы, однако, не будем использовать алгебраический подход - решать соответствуюшее дифференциальное уравнение ${ }^{32}$ ), а вместо этого используем язык теории групп. А именно, мы найдем функции $f_{L, \lambda}(g)$ и $f_{R, \lambda}(g)$ в пространстве представления, удовлетворяющие условиям

$$
\begin{gathered}
\pi_{\lambda}(z) f_{R, \lambda}(g)=f_{R, \lambda}(g z)=e^{\sum i \mu_{i} z_{i, i+1}} f_{R, \lambda}(g)=e^{i \operatorname{Tr}(\mu z)} f_{R, \lambda}(g), \\
(\mu)_{i j}=\delta_{i-1, j} \mu_{i}, \quad g, z \in N_{+}
\end{gathered}
$$

и

$$
\pi_{\lambda}\left(z^{t}\right) f_{L, \lambda}(g)=e^{i \operatorname{Tr}\left(\mu_{L} z\right)} f_{L, \lambda}(g),
$$

являющимся групповым аналогом условий (П4.10) и (П4.11).

Решение первого условия эквивалентно построению одномерного представления группы верхнетреугольных матрищ. Вначале мы сконструируем аддитивный характер этой группы, используя тот факт, что в произведении двух верхнетреугольных матриц

${ }^{32)}$ В [39] описано, как это делать в данном случае. 
их элементы, лежащие на следующей за основной диагонали, складьваются. Тогда можно экспоненцировать этот характер, получая искомое одномерное представление

$$
f_{R, \lambda}^{\mu}(x)=e^{i \operatorname{Tr}(\mu x)}
$$

Для нахождения функции $f_{L, \lambda}(x)$, удовлетворяющей уравнению (П4.15), мы используем внутренний автоморфизм группы $S L(p)$, отображающий строго верхнетреугольные матрицы в строго нижнетреугольные. Этот автоморфизм явно описывается матрицей

$$
S=\left(\begin{array}{ccccccc}
0 & 0 & 0 & 0 & 0 & 0 & 1 \\
0 & \ldots & 0 & 0 & 0 & 1 & 0 \\
0 & \ldots & 0 & 0 & 1 & 0 & 0 \\
0 & \ldots & 0 & 1 & 0 & \ldots & 0 \\
& \ldots & 1 & 0 & 0 & \ldots & \\
0 & 1 & 0 & \ldots & 0 & \ldots & 0 \\
1 & \ldots & 0 & 0 & 0 & \ldots & 0
\end{array}\right)
$$

т.е.

$$
S_{i j}=\delta_{i+j, p+1}
$$

и

$$
\left(S^{-1} z S\right)_{i j}=z_{p+1-i, p+1-j}
$$

На самом деле, необходимо, чтобы матрица $S$ была элементом группы $S O(p)$ (в общем случае она конструируется как элемент группы Вейля [39]). Следовательно, она должна быть нормирована так, чтобы иметь единичный детерминант. Это может быть сделано домножением на -1 , что не влияет на формулу (П4.19). Заметим, что автоморфизм $S$ отражает элемент $z_{i, i-1}$ в элемент $z_{p-i, p+1-i}$ вместо $z_{i-1, i}$ (из-за условия (П4.11) нам интересны только элементы $z$, лежащие на ближайшей к основной диагонали). Ситуация может быть исправлена подходящим отражением матрицы $\mu$. А именно, нужно ввести новую матрицу $\tilde{\mu}_{L} \equiv \mu_{p-i}^{L} \delta_{i-1, j}$, после чего условие (П4.15) может быть переписано в виде

$$
\pi_{\lambda}\left(S z S^{-1}\right) f_{L}(g)=f_{L}\left(g S z S^{-1}\right) e^{i \operatorname{Tr}\left(\tilde{\mu}_{L} z\right)} f_{L, \lambda}\left(g S^{-1} S\right)
$$

т.е.

$$
f_{L}\left(g S^{-1} z\right)=e^{i \operatorname{Tr}\left(\tilde{\mu}_{L} z\right)} f_{L, \lambda}\left(g S^{-1}\right) .
$$

Как мы уже видели, решение такого уравнения в верхнетреугольных матрицах имеет вид

$$
\left.f_{L}\left(g S^{-1}\right)\right|_{B_{-}=0}=f_{L}\left(n_{+}\right)=e^{i \operatorname{Tr}\left(\tilde{\mu}_{L} n_{+}\right)}
$$

где $n_{+}-$верхнетреугольная часть $g S^{-1}$. Однако нам нужно решение (П4.20) для $g=$ $x \in N_{+}$, поэтому требуется некоторый пересчет:

$$
f_{L}\left(x S^{-1}\right)=\chi_{\lambda}\left(x S^{-1}\right) f_{L}\left(n_{+}\right)=\chi_{\lambda}\left(x S^{-1}\right) e^{i \operatorname{Tr}\left(\tilde{\mu}_{L} n_{+}\right)}
$$


Вычислим эту функцию более явно. Прежде всего элементы диагональной матрицы $h$ в разложении Гаусса матришы $g$ даются формулой ${ }^{33)}$

$$
h_{i}=\frac{\Delta_{i}(g)}{\Delta_{i-1}(g)}, \quad h_{1}=\Delta_{1}(g), \quad \Delta_{p}(g)=\frac{\Delta_{1}(g)}{\Delta_{p-1}(g)},
$$

где $\Delta_{i}(g)$ обозначает верхний главный минор матрицы $g$ размера $i \times i$. Таким образом, диагональная часть $x S^{-1}$ дается отношением $h_{i}=\Delta_{i}\left(x S^{-1}\right) / \Delta_{i-1}\left(x S^{-1}\right)$ и

$$
\chi_{\lambda}\left(x S^{-1}\right)=\prod_{i} \Delta_{i}^{\left(\boldsymbol{\lambda} \boldsymbol{\alpha}_{i}-1\right)}\left(x S^{-1}\right) .
$$

Чтобы получить формулу для элементов $\left(n_{+}\right)_{k-1, k}$, заметим, что они зависят только от подматрицы размера $k \times k$ в левом верхнем углу. Рассмотрим элемент $(k-1, k)$ матрицы $b^{-1} \equiv n_{+} g^{-1}, g=x S^{-1}$. Он равен нулю по определению $b$, так что мы получаем тождество

$$
\left(n_{+}\right)_{k-1, k}\left(g^{-1}\right)_{k, k}+\left(n_{+}\right)_{k-1, k-1}\left(g^{-1}\right)_{k-1, k}=0 .
$$

Используя явное представление для элементов обратной матрицы, находим желаемое выражение:

$$
\left(n_{+}\right)_{k-1, k}=\frac{\Delta_{k-1, k}\left(x S^{-1}\right)}{\Delta_{k-1}\left(x S^{-1}\right)},
$$

где символ $\Delta_{k-1, k}\left(x S^{-1}\right)$ определен как детерминант подматрицы размера $(k-1) \times$ $(k-1)$ матрищы $x S^{-1}$ с переставленными $k$ и $k-1$ столбцами.

Таким образом, мы получили явные выражения для функций $f_{L}$ и $f_{R}$. Для вычисления функции Уиттекера нам теперь осталось определить действие картановской части группового элемента $g$ на $f_{R}(x)$. Заметим, что, хотя элемент $x h$ с $x \in X$ и $h \in H$ не принадлежит $X$, элемент $h^{-1} x h$ принадлежит. Поэтому с помощью (П4.6) можно получить

$$
\pi_{\lambda}(h) f_{R, \lambda}(x)=f_{R, \lambda}\left(h h^{-1} x h\right)=h^{\boldsymbol{\lambda}-\boldsymbol{\rho}} f_{R, \lambda}\left(h^{-1} x h\right) .
$$

Теперь, используя эту формулу и явное выражение (П4.16), мы окончательно получаем

$$
\begin{aligned}
\pi_{\lambda}\left(e^{-\boldsymbol{\mu}_{i} \boldsymbol{\phi} T_{0, i}}\right) f_{R, \lambda}(x) & =e^{\left\{-\boldsymbol{\mu}_{i} \cdot \boldsymbol{\phi}\right\}\left\{(\boldsymbol{\lambda}-\boldsymbol{\rho}) \cdot\left(\mathbf{e}_{i+1}-\mathbf{e}_{i}\right)\right\}} f_{R, \lambda}\left(e^{\boldsymbol{\mu}_{i} \boldsymbol{\phi} T_{0, i}} x e^{-\boldsymbol{\mu}_{i} \boldsymbol{\phi} T_{0, i}}\right)= \\
& =e^{(\boldsymbol{\rho}-\boldsymbol{\lambda}) \boldsymbol{\phi}} f_{R, \lambda}\left(e^{\boldsymbol{\mu}_{i} \boldsymbol{\phi} T_{0, i}} x e^{-\boldsymbol{\mu}_{i} \boldsymbol{\phi} T_{0, i}}\right)= \\
& =e^{(\boldsymbol{\rho}-\boldsymbol{\lambda}) \boldsymbol{\phi}} e^{i \operatorname{Tr} x \mu e^{\boldsymbol{\alpha} \boldsymbol{\phi}}},
\end{aligned}
$$

где комбинация $\mu e^{\boldsymbol{\alpha} \boldsymbol{\phi}}$ обозначает матрицу с матричными элементами $\delta_{i-1, j} \mu_{i} e^{\boldsymbol{\alpha}_{i} \boldsymbol{\phi}}$. Собирая все это вместе, мы находим окончательный результат для функции Уиттекеpa $(6.42)$

$$
\begin{aligned}
\Psi(\phi)= & e^{-\boldsymbol{\lambda} \boldsymbol{\phi}} \int_{X=B \backslash G} \prod_{i<j} d x_{i j} \prod_{i=1}^{p-1} \Delta_{i}^{-\left(\boldsymbol{\lambda} \boldsymbol{\alpha}_{i}+1\right)}\left(x S^{-1}\right) \times \\
& \times \exp \left\{i \mu_{i}^{R} x_{i, i+1} e^{\boldsymbol{\alpha}_{i} \boldsymbol{\phi}}-i \mu_{p-i}^{L} \frac{\Delta_{i, i+1}\left(x S^{-1}\right)}{\Delta_{i}\left(x S^{-1}\right)}\right\} .
\end{aligned}
$$

\footnotetext{
33) Проще всего вывести эту формулу, заметив, что диагональный элемент $g_{i i}$ матрицы $g$ зависит только от матричных элементов, лежащих в левом верхнем углу в подматрице размера $i \times i$. Тогда, используя индукцию по рангу матрицы, мы можем получить формулу (П4.24).
} 


\section{Список литературы}

[1] A. Мариаков. Точные непертурбативные решения квантовой теории и интегрируемые системы. Дисс. на соискание уч. ст. докт. физ.-матем. наук. М.: ФИАН, 1997.

[2] A. Миронов. $\tau$-Функции и матричные модели. Дисс. на соискание уч. ст. докт. физ.-матем. наук. М.: ФИАН, 1997.

[3] А. Б. Замолодчиков. Письма в ЖЭТФ. 1987. Т. 46. С. 129-132.

[4] B. A. Kupershmidt, P. Mathieu. Phys. Lett. B. 1989. V. 227. P. 245.

[5] T. Eguchi, S. K. Yang. Phys. Lett. B. 1989. V. 244. P. 373-378.

[6] A. B. Zamolodchikov. Adv. Stud. in Pure Math. 1989. V. 19. P. 641-674.

[7] R. Sasaki, I. Yamanaka. Adv. Stud. in Pure Math. 1988. V. 16. P. 271-296.

[8] А. Морозов. УФН. 1992. Т. 162. № 8. С. 84.

[9] В. Книжник. УФН. 1989. Т. 159. № 3. С. 401-453.

[10] S. Saito. Phys. Rev. Lett. 1987. V. 59. P. 1798.

[11] S. Saito. Phys. Rev. D. 1987. V. 36. P. 1819.

[12] A. Morozov. Phys. Lett. B. 1987. V. 196. P. 325.

[13] A.R. Its, A. G. Izergin, V. E. Korepin, N. A. Slavnov. Int. J. Mod. Phys. B. 1990. V. 4. P. $1003-1037$.

[14] М. Сато, М. Джимбо, Т. Мива. Голономные квантовые поля. М.: Мир, 1983.

[15] D. Bernard, A. Le Clair. Differential Equations for Sine-Gordon Correlation Functions at the Free Fermion Point. Preprint CLNS 94/1276, SPhT-94-021; hep-th/9402144.

[16] A. G. Izergin, D. A. Coker, V. E. Korepin. J. Phys.: Math.Gen. A. 1992. V. 25. P. 4315-4334.

[17] А. Морозов. УФН. 1994. Т. 164. № 1. С. 3-62.

[18] A. Marshakov. Int. J. Mod. Phys. A. 1993. V. 8. № 22. P. 3831-3882.

[19] A. Mironov. Int. J. Mod. Phys. A. 1994. V. 9. P. 4355-4405.

[20] N. Seiberg, E. Witten. Nucl. Phys. B. 1994. V. 426. P. 19-52.

[21] N. Seiberg, E. Witten. Nucl. Phys. B. 1994. V. 431. P. 484-550.

[22] A. Gorsky, I. Krichever, A. Marshakov, A. Mironov, A. Morozov. Phys. Lett. B. 1995. V. 355. P. $466-474$.

[23] A. Mironov. WDVV equations in Seiberg-Witten theory and associative algebras. hep-th/9704205.

[24] A. Gorsky, S. Gukov, A. Mironov. Multiscale $N=2$ SUSY field theories, integrable systems and their stringy/brane origin. hep-th/9707120.

[25] Л. А. Тахтаджсян, Л. Д. Фаддеев. УМН. 1979. Т. 34. С. 13-63.

[26] Н. Ю. Решетихин, Л. А. Тахтаджян, Л.Д. Фаддеев. Алгебра и анализ. 1989. Т. 1. C. $178-206$.

[27] Н. М. Боголюбов, А.Г. Изергин, В.Е. Корепин. Корреляционные функции интегрируемых систем и квантовый метод обратной задачи. М.: Наука, 1992.

[28] Р. Бәкстер. Точно решаемые модели в статистической механике. М.: Мир, 1985.

[29] L. D. Faddeev. Integrable models in 1+1-dimensional quantum field theory. In: Les Houches lectures 1982. Amsterdam: Elsevier, 1984. P. 563-608.

[30] E. Sklyanin. Quantum inverse scattering method. Selected topics. In: Quantum group and quantum integrable systems (Nankai lectures in mathematical physics). Ed. Mo-Lin Ge. Singapore: World Scientific, 1992. P. 63-97.

[31] S. Kharchev, A. Marshakov, A. Mironov, A. Morozov. Int. J. Mod. Phys. A. 1995. V. 10. P. $2015-2052$

[32] K. Wilson. Phys. Rev. D. 1974. V. 10. P. 2445.

[33] G. Weight. On Operator Constructions in Bosonic Liouville Quantum Gravity. In: Proceedings of the 28th International Simposium Ahrenshoop. Wendisch-Rietz, Germany, September 1993. Hamburg: DASY, 1994

[34] А. Д. Миронов, А. Ю. Морозов, Л. Винэ. ТМФ. 1994. Т. 100. № 1. С. 119-131.

[35] A. Gerasimov, S. Khoroshkin, D. Lebedev, A. Mironov, A. Morozov. Int. J. Mod. Phys. A. 1995. V. 10. P. 2589-2614.

[36] A. Mironov. CRM Proc. Lect. Notes. 1996. V. 9. P. 219-237. 
[37] S. Kharchev, A. Mironov, A. Morozov. $\tau$-Functions: Evolution and Determinant Representations. In: Proceedings of the XXVIII International Symposium Arenshoop, 1995. Hamburg: DASY, 1995. P. 80-89.

[38] С. М. Харчев, А. Д. Миронов, А. Ю. Морозов. ТМФ. 1995. Т. 104. № 1. С. 129-143.

[39] A. Gerasimov, S. Kharchev, A. Marshakov, A. Mironov, A. Morozov, M. Olshanetsky. Liouville type models in group theory framework. I. Finite-dimensional algebras. Preprint FIAN/TD-18/95, ITEP M4/TH-7/95; hep-th/9601161.

[40] A. Mironov. Group theory structures underlying integrable systems. Preprint FIAN/TD-12/96, ITEP/TH-24/96; hep-th/9607123; Proc. 2nd International A.D. Sakharov Conference. Singapore: Science, 1997.

[41] M. Olshanetsky, A. Perelomov. Invent. Math. 1979. V. 54. P. 261-269.

[42] B. Kostant. Adv. Math. 1979. V. 34. P. 195-338.

[43] М. А. Ольианецкий, А. М. Переломов. ТМФ. 1980. Т. 45. № 1. С. 3-18.

[44] Н. Харт. Геометрическое квантование в действии. М.: Мир, 1985.

[45] H. P. B. Jacquet. Bull. Soc. Math. France. 1967. V. 95. P. 243-309.

[46] G. Schiffmann. Bull. Soc. Math. France. 1971. V. 99. P. 3-72.

[47] B. Kostant. Invent. Math. 1978. V. 48. P. 101-184.

[48] M. Hashizume. Hiroshima Math. J. 1982. V. 12. P. 259-293.

[49] С.Г. Гиндикин, Ф. И. Карпелевич. Докл. АН СССР. 1962. Т. 145. С. 252-255.

[50] С. Г. Гиндикин, Ф. И. Карпелевич. Изв. АН СССР, сер. матем. 1966. Т. 30. С. 1147-1156.

[51] М. С. Семенов-Тян-Шанский. Квантование открытых цепочек Тоды. В сб.: Современные проблемы математики. Т. 16. М.: ВИНИТИ, 1987. С. 194-226.

[52] A. Gerasimov, A. Marshakov, A. Morozov, M. Olshanetsky, S. Shatashvili. Int. J. Mod. Phys. A. 1990. V. 5. P. 2495-2589.

[53] A. Zamolodchikov, Al.Zamolodchikov. Structure constants and conformal bootstrap in Liouville field theory. hep-th/9506136.

[54] H. Dorn, H.-J. Otto. Phys. Lett. B. 1992. V. 291. P. 39-43.

[55] H. Dorn, H.-J. Otto. Nucl. Phys. B. 1994. V. 429. P. 375-388.

[56] M. Kashiwara, T. Miwa. Proc. Japan Acad. A. 1981. V. 57. P. 342-347.

[57] E. Date, M. Kashiwara, T. Miwa. Proc.Japan Acad. A. 1981. V. 57. P. 387-392.

[58] E. Date, M. Jimbo, M. Kashiwara, T. Miwa. J. Phys. Soc. Japan. 1981. V. 50. P. 3806-3812.

[59] E. Date, M. Jimbo, M. Kashiwara, T. Miwa. Physica. D. 1982. V. 4. P. 343-365.

[60] E. Date, M. Jimbo, M. Kashiwara, T. Miwa. Publ. RIMS Kyoto Univ. 1983. V. 18. P. 1111-1119.

[61] E. Date, M. Jimbo, M. Kashiwara, T. Miwa. J. Phys. Soc. Japan. 1981. V. 50. P. 3813-3818.

[62] E. Date, M. Jimbo, M. Kashiwara, T. Miwa. Transformation groups for soliton equations. In: Proc. RIMS symp. Nonlinear integrable systems - classical theory and quantum theory. Eds M. Jimbo, T. Miws. Singapore: World Scientific, 1983. P. 39.

[63] M. Jimbo, T. Miwa. Publ. RIMS Kyoto Univ. 1983. V. 19. P. 943-1001.

[64] K. Ueno, K. Takasaki. Adv. Studies in Pure Math. 1984. V. 4. P. 1-95.

[65] S. Kharchev, A. Marshakov, A. Mironov, A. Morozov. Nucl. Phys. B. 1993. V. 397. P. $339-378$.

[66] M. Sato. RIMS Kokyuroku. 1981. V. 439. P. 30-40.

[67] И. М. Кричевер. Функц. анализ и его прилож. 1977. Т. 11. С. 15-31.

[68] И. М. Кричевер. УМН. 1977. Т. 32. С. 183-208.

[69] G. Segal, G. Wilson. Publ. I.H.E.S. 1985. V. 61. P. 5-65.

[70] В.Е. Захаров, С. В. Манаков, С. П. Новиков, Л. П. Питаевский. Теория солитонов. Метод обратной задачи. М.: Наука, 1980.

[71] V. Kă̌, A. S. Schwarz. Phys. Lett. B. 1991. V. 257. P. 329.

[72] S. Kharchev, A. Marshakov, A. Mironov, A. Morozov, A. Zabrodin. Nucl. Phys. B. 1992. V. 380. P. 181-240.

[73] S. Kharchev, A. Marshakov, A. Mironov, A. Orlov, A. Zabrodin. Nucl. Phys. B. 1991. V. 366. P. 569-601.

[74] P. J. Hansen, D. J. Kaup. J. Phys. Soc. Japan. 1985. V. 54. P. 4126.

[75] M. A. Olshanetsky, A. M. Perelomov. Phys. Rep. 1981. V. 71. P. 313-400. 
[76] M. Olshanetsky, A. Perelomov. Phys. Rep. 1983. V. 94. P. 313-404.

[77] O. I. Bogoyavlensky. Commun. Math. Phys. 1976. V. 51. P. 201-209.

[78] R. Hirota. J. of Phys. Soc. Japan. 1987. V. 57. P. 4285-4288.

[79] R. Hirota, Y. Ohta, J. Satsuma. Progr. Theor. Phys. Suppl. 1988. V. 94. P. 59-72.

[80] A. Leznov, M. Saveliev. Physica. D. 1981. V. 3. P. 62-72.

[81] A. Н. Лезнов, М. В. Савельев. Методы теории групп интегрирования нелинейных динамических систем. М.: Наука, 1985.

[82] O. Babelon. Commun. Math. Phys. 1991. V. 139. P. 619-643.

[83] O. Babelon. Phys. Lett. B. 1988. V. 215. P. 523.

[84] J.-L. Gervais. Nucl. Phys. B. 1993. V. 391. P. 287-332.

[85] J.-L. Gervais, J. Schnittger. Nucl. Phys. B. 1994. V. 431. P. 273-314.

[86] Й. Шнитгер. ТМФ. 1995. Т. 104. № 1. С. 158-191.

[87] I. Frenkel, N. Reshetikhin. Commun. Math. Phys. 1992. V. 146. P. 1.

[88] M. Jimbo, T. Miwa. Algebraic Analysis of Solvable Lattice Models. Preprint RIMS-981, May 1994. Kioto: Kioto Univ., 1994

[89] C. Chari, A. Pressley. A guide to quantum groups. Cambridge: Cambridge University Press, 1994.

[90] C. Fronsdal, A. Galindo. The Universal T-Matrix. Preprint UCLA/93/TEP/2, January 1993.

[91] A. Morozov, L. Vinet. Free-field representation of group element for simple quantum groups. Preprint ITEP-M3/94, CRM-2202; hep-th/9409093.

[92] Д. П. Желобенко. Компактные группы Ли и их представления. М.: Наука, 1970.

[93] Л. Л. Ваксман, Я. С. Сойбельман. Функц. анализ и его прилож. 1988. Т. 23. С. 1-14.

[94] S. Levendorskii, Y. Soibelman. Commun. Math. Phys. 1991. V. 139. P. 141-170.

[95] Y. Soibelman. Int. Math. Research Notices. 1993. № 6. P. 151-163.

[96] K. Kajiwara, J. Satsuma. J. Phys. Soc. Jpn. 1991. V. 60. P. 3986-3989.

[97] K. Kajiwara, Y. Ohta, J. Satsuma. $q$-Discrete Toda molecule equation. solv-int/9304001.

[98] M. Rosso. Commun. Math. Phys. 1988. V. 117. P. 581-593.

[99] A. М. Переломов. Интегрируемые системы классической механики и алгебры Ли. М.: Наука, 1990

[100] Л. Д. Фаддеев, Л. А. Тахтаджкян. Гамильтонов подход в теории солитонов. М.: Наука, 1986 .

[101] M. Olshanetsky, V. Rogov. J. Phys.: Math. Gen. 1994. V. 27. P. 4669-4683.

[102] И. М. Гельфанд, М. И. Граев, И. И. Пятецкий-Шапиро. Теория представлений и автоморфные функции. М.: Наука, 1966.

[103] И. С. Градштейн, И. М. Рыжик. Таблицы интегралов, сумм, рядов и произведений. М.: Физматгиз, 1963.

[104] Harish-Chandra. Amer. J. Math. 1958. V. 80. P. 241-310, 553-613.

[105] Д. Мамфорд. Лекции о тэта-функциях. М.: Мир, 1988.

[106] H. Awata, M. Noumi, S. Odake. Heisenberg realization for $U_{q}(S L(n))$ on the flag manifold. Preprint YITP/K-1016, May 1993; hep-th/9306010.

[107] P. Griffits, F. Adams. Topics in algebraic geometry and analytic geometry. In: Mathematical Notes. V. 13. Princeton: Princeton University Press, 1974.

[108] В. Г. Дринфельд. Зап. научн. семин. ЛОМИ. 1986. Т. 155. С. 19-49.

[109] M. Jimbo. Lett. Math. Phys. 1985. V. 10. P. 63-69.

[110] N. Wallach. Harmonic Analysis in Homogeneous Spaces. New York: Dekker, 1973.

[111] С. Хелгасон. Группы и геометрический анализ. М.: Мир, 1987. 